\title{
An observational study of dust nucleation in Mira (o Ceti)
}

\section{Titanium oxides are negligible for nucleation at high temperatures}

\author{
T. Kamiński ${ }^{1,2}$, H. S. P. Müller ${ }^{3}$, M. R. Schmidt ${ }^{4}$, I. Cherchneff ${ }^{5}$, K. T. Wong (黃嘉達) ${ }^{6}$, S. Brünken ${ }^{3}$, K. M. Menten ${ }^{6}$, \\ J. M. Winters ${ }^{7}$, C. A. Gottlieb ${ }^{2}$, and N. A. Patel ${ }^{2}$
}

\author{
1 ESO, Alonso de Cordova 3107, Vitacura, 19001 Casilla, Santiago, Chile \\ e-mail: tomasz.kaminski@cfa.harvard.edu \\ 2 Harvard-Smithsonian Center for Astrophysics, 60 Garden Street, Cambridge, MA, USA \\ 3 I. Physikalisches Institut, Universität zu Köln, Zülpicher Strasse 77, 50937 Köln, Germany \\ ${ }_{4}$ Nicolaus Copernicus Astronomical Center, Polish Academy of Sciences, Rabiańska 8, 87-100 Toruń, Poland \\ 5 Departement Physik, Universität Basel, Klingelbergstrasse 82, 4056 Basel, Switzerland \\ 6 Max-Planck-Institut für Radioastronomie, Auf dem Hügel 69, 53121 Bonn, Germany \\ 7 IRAM, 300 rue de la Piscine, Domaine Universitaire de Grenoble, 38406 St.-Martin-d'Hères, France
}

Received 4 October 2016 / Accepted 9 November 2016

\begin{abstract}
Context. The formation of silicate dust in oxygen-rich envelopes of evolved stars is thought to be initiated by the formation of seed particles that can withstand the high temperatures close to the stellar photosphere and act as condensation cores farther away from the star. $\mathrm{TiO}$ and $\mathrm{TiO}_{2}$ are among the candidate species considered as first condensates.

Aims. We aim to identify and characterize the circumstellar gas-phase chemistry of titanium that leads to the formation of solid titanium compounds in the envelope of $o$ Ceti, the prototypical Mira, and seek an observational verification of whether titanium oxides play a major role in the onset of dust formation in M-type asymptotic giant branch (AGB) stars.

Methods. We present high angular resolution (145 mas) ALMA observations at submillimeter (submm) wavelengths supplemented by APEX and Herschel spectra of the rotational features of $\mathrm{TiO}$ and $\mathrm{TiO}_{2}$. In addition, circumstellar features of $\mathrm{TiO}$ and $\mathrm{TiI}$ are identified in optical spectra, which cover multiple pulsation cycles of $o$ Ceti.

Results. The submm ALMA data reveal $\mathrm{TiO}$ and $\mathrm{TiO}_{2}$ bearing gas within the extended atmosphere of Mira. While TiO is traceable up to a radius $(F W H M / 2)$ of 4.0 stellar radii $\left(R_{\star}\right), \mathrm{TiO}_{2}$ extends as far as $5.5 R_{\star}$ and, unlike $\mathrm{TiO}$, appears to be anisotropically distributed. Optical spectra display variable emission of Ti I and TiO from inner parts of the extended atmosphere $\left(<3 R_{\star}\right)$.

Conclusions. Chemical models that include shocks are in general agreement with the observations of gas-phase, titanium-bearing molecules. It is unlikely that substantial amounts of titanium is locked up in solids because the abundance of the gaseous titanium species is very high. The formation of hot titanium-rich condensates is very improbable because we find no traces of their hot precursor species in the gas phase. It therefore appears unlikely that the formation of dust in Mira, and possibly other M-type AGB stars, is initiated by titanium oxides.
\end{abstract}

Key words. stars: mass-loss - circumstellar matter - astrochemistry - stars: individual: $o$ Ceti - submillimeter: stars

\section{Introduction}

Asymptotic giant branch (AGB) stars lose a significant part of their mass through stellar winds that are rich in dust (e.g., Gehrz 1989; Gail et al. 2009). The dust enters the interstellar medium where it is processed and later becomes an important ingredient of the material from which new stars and planets form. In a galaxy like ours, AGB stars contribute the most mass to the overall dust production, mostly in the form of carbonaceous and silicate dust (e.g., Ferrarotti \& Gail 2006). Silicate-dust production is initiated in oxygen-rich (O-rich) stars by the formation of condensates of small metal oxides; the elemental abundance of oxygen is higher than that of carbon in O-rich stars. Based on infrared interferometric observations, the transition from molecular to solid form must occur at distances of $\lesssim 2$ stellar radii (e.g., Danchi et al. 1994; Norris et al. 2012; Karovicova et al. 2013; Zhao-Geisler et al. 2012). Because temperatures in this region are high (roughly 1000-2500 K), these first condensates must be formed from very refractory species. In most theoretical studies attempting to explain the nucleation process, it has been assumed that circumstellar molecules and dust form under chemical- and thermal-equilibrium (TE) conditions (Gail \& Sedlmayr 1986, 1998; Tielens 1991; Jeong et al. 2003). These models indicate that the dust production is likely taking place through heterogeneous nucleation. In this scenario, the first hot condensates are produced from inorganic material other than silicates. At lower temperatures, roughly $<600 \mathrm{~K}$, they act as condensation cores (seeds) that facilitate subsequently more efficient growth of silicates (e.g., Höfner et al. 2016). Likely candidates for the first condensates are titanium oxides (mainly $\mathrm{TiO}_{2}$ ) and alumina dust $\left(\mathrm{Al}_{2} \mathrm{O}_{3}\right.$; e.g., Gail \& Sedlmayr 1998; Jeong et al. 2003). However, because atmospheres of AGB stars are affected by shock waves triggered by pulsations, it is questionable whether the equilibrium conditions can prevail in the dust-formation regions (Willacy \& Cherchneff 1998; Cherchneff 2006). In consequence, it has been unclear whether the heterogeneous nucleation indeed takes place around pulsating AGB stars. In recent years, however, the role of titanium and aluminum oxides as seeds can be tested observationally. The gas-phase precursors of these solids can now be observed directly by optical, infrared, 
and millimeter/submillimeter ( $\mathrm{mm} / \mathrm{submm})$ spectroscopy. In Kamiński et al. (2016; hereafter Paper I), we performed a first observational verification of whether aluminum-bearing species that are necessary precursors of alumina seeds are present in O-rich AGB stars. Although a full quantitative analysis was not possible, all observations of the prototypical M-type AGB star, $o$ Ceti, are consistent with aluminum depletion within 2-4 stellar radii. However, not all $\mathrm{Al}$ is consumed from the gas phase in the dust-formation region and the $\mathrm{Al}$ depletion is anisotropic. In this paper, we focus on the role of titanium oxides in the formation of dust at high temperatures.

The important role of titanium oxides, particularly of $\mathrm{TiO}_{2}$, for inorganic dust formation has been chiefly promoted on theoretical grounds (e.g., Gail \& Sedlmayr 1998; Jeong et al. 2003, 1999). There is also observational evidence that Ti species are involved in nucleation. Studies of presolar grains show that, although scarce, titanium oxide grains form in AGB winds (e.g., Nittler et al. 2008). It has also been long known that titanium is considerably depleted in the interstellar medium presumably because it is effectively locked into dust grains (Stokes 1978; Churchwell et al. 1980). Titanium oxides are also considered to play an important role in the condensation of solids in the atmospheres of planets and brown dwarfs, although in different temperature and pressure regimes than prevailing around evolved stars (e.g., Burrows \& Sharp 1999; Lee et al. 2015).

On the other hand, the role of titanium oxides in the seed formation process has often been questioned, mainly because titanium is not an abundant metal. Titanium is 35 times less abundant than aluminum, whose production of refractory alumina dust is an alternative scenario to Ti seeds. Very efficient nucleation of $\mathrm{TiO}_{2}$ would be necessary for titanium oxides to be responsible for the observed dust production rates in O-rich evolved stars (Jeong et al. 1999). Moreover, solid titanium compounds have not been conclusively identified in stellar spectra, although optical constants have been measured for a number of these compounds; these include for example, $\mathrm{TiO}_{2}, \mathrm{CaTiO}_{3}$, and $\mathrm{MgTiO}_{3}$ (Posch et al. 2003; Tamanai et al. 2009). Studies of presolar grains do not identify titanium compounds as forming the grain cores that would be expected in a heterogeneous condensation sequence (Stroud et al. 2004; Nittler et al. 2008). Moreover, Kamiński et al. (2013) showed that Ti condensation is inefficient in some circumstellar environments. They identified emission features of $\mathrm{Ti}$ I, $\mathrm{TiO}$, and $\mathrm{TiO}_{2}$ in the spectra of the red supergiant VY CMa and found very high abundances of the gas-phase species at large distances from the star. These results are in stark contrast with the nucleation scenario in which titanium oxides play a major role in the dust production close to the star (see also De Beck et al. 2015). It was realized, however, that the environment of VY CMa may be too violent to be considered representative for the majority of Galactic dust producers, such as AGB stars (Kamiński et al. 2013).

It was recently proposed that the onset of dust formation may be controlled solely by SiO (Gail et al. 2013, 2016). Revised laboratory data extrapolated to conditions characteristic of the upper atmosphere indicate that $\mathrm{SiO}$ seeds can grow under equilibrium conditions at higher temperatures (but still $\lesssim 800 \mathrm{~K}$ ) than it was assumed when the heterogeneous nucleation scenario was first introduced (Gail et al. 2013, and references therein). Models based on chemical kinetics show, however, that the formation of dust is induced by non-equilibrium processes pertaining to the shocked gas and that dimerization of $\mathrm{SiO}$ under non-equilibrium conditions is very slow (Cherchneff 2006, 2012; Gobrecht et al. 2016; Bromley et al. 2016). Observational test of the heterogeneous nucleation is necessary.
In a parallel approach to Paper I (see also Kamiński et al. 2013), we attempt here to characterize all accessible gas-phase titanium-bearing species in the circumstellar envelope of $o$ Ceti to test whether they are efficiently depleted into solids and whether they are the precursors of the first condensates in the winds of M-type stars. An introduction to the nucleation process, a description of the structure of a circumstellar envelope of an AGB star, and a characterization of our primary target, Mira, are given in Paper I and are not repeated here. In Sect. 2, we first review all known Ti tracers and identify species of our main interest. The observational material is briefly presented in Sect. 3 and includes mm/submm data (Sect. 3.1) and multi-epoch optical spectra (Sect. 3.2). To provide a context to these observations, in Sect. 4 we briefly analyze the state and structure of Mira and its extended atmosphere at the time of the observations. Then, in Sect. 5, the Ti-bearing gas is characterized based on submm/mm features of $\mathrm{TiO}$ and $\mathrm{TiO}_{2}$. In the following Sect. 6, we identify and analyze optical signatures of Ti-bearing gas, including those of $\mathrm{Ti}$ II, Ti I, TiO, and $\mathrm{TiO}_{2}$. The relevance of the results on the chemistry and dust nucleation in M-type AGB stars is discussed in Sect. 7.

\section{Ti-bearing species in circumstellar envelopes}

The first solid particles based on titanium oxides are thought to be $\mathrm{TiO}_{2}$ (titania) and can be followed by formation of higher oxides, including $\mathrm{Ti}_{2} \mathrm{O}_{3}, \mathrm{Ti}_{3} \mathrm{O}_{5}$, and $\mathrm{Ti}_{4} \mathrm{O}_{7}$. Other titanium compounds, for example, $\mathrm{CaTiO}_{3}$ (perovskite) and $\mathrm{MgTiO}_{3}$, are also potentially produced by evolved stars (Sharp \& Huebner 1990; Gail \& Sedlmayr 1998; Plane 2013). However, the formation of solids from $\mathrm{TiO}_{2}$ clusters is considered the simplest plausible scenario for grain growth, in particular because $\mathrm{TiO}_{2}$ is thought to be present in the gas phase. $\mathrm{TiO}_{2}$ has several natural solid forms, for example crystalline anatase, brookite, and rutile. Those solids are formed directly out of the gaseous TiO. The solid form of $\mathrm{TiO}$ also exists and can be produced in a reaction of $\mathrm{H}_{2}$ with gaseous $\mathrm{TiO}_{2}$. The $\mathrm{TiO}_{2}$ gas, in turn, forms directly from $\mathrm{TiO}$ in a reaction with $\mathrm{OH}$ (Plane 2013; Gobrecht et al. 2016) and with water (Gail \& Sedlmayr 1998), while $\mathrm{TiO}$ is formed from atomic titanium, Ti I. Atomic titanium can exist in neutral and ionic forms in the atmospheres and envelopes of cool evolved stars. In chemical equilibrium, the ionization fraction is mainly dependent on the photospheric temperature (e.g., Tsuji 1973), but in real pulsating stars this fraction can be strongly influenced by the shock strength and level of penetration by the shock-induced ultraviolet radiation. The equilibrium chemistry of the Ti-bearing species is discussed in great detail in Gail \& Sedlmayr (1998).

In order to trace the different chemical forms of titanium in the gas phase, we aim to identify and characterize features of atomic titanium and the two simplest oxides, $\mathrm{TiO}$ and $\mathrm{TiO}_{2}$. The electronic bands of $\mathrm{TiO}$ have long been known in the photospheres of late-type O-rich stars but only a few astronomical objects manifested the presence of circumstellar TiO-bearing gas in those bands (Kamiński et al. 2013, and references therein). In Sect.6, we attempt to identify all forms of circumstellar Ti-bearing species in the optical spectra of Mira. Millimeter/submillimeter observations of circumstellar $\mathrm{TiO}$ and $\mathrm{TiO}_{2}$, through their pure rotational lines, are also very rare and have become possible only recently (Kamiński et al. 2013). We successfully identify the rotational spectra of both species in Mira in Sect. 5. In addition, TiO has its fundamental vibration band near $10 \mu \mathrm{m}$, which so far have been identified in one S-type AGB star, NP Aur (Smolders et al. 2012). Owing to their extreme rarity, the 
mid-infrared bands do not seem to be a good tracer of $\mathrm{TiO}$ in stars. Additionally, in observations that do not spatially resolve the extended atmosphere of Mira, the TiO feature would be completely buried under the much stronger silicate feature. We therefore do not attempt to trace $\mathrm{TiO}$ in these infrared bands.

In addition to the oxides and atomic forms, the hydride, $\mathrm{TiH}$, and simple compounds of other abundant metals, for example, $\mathrm{TiN}, \mathrm{TiS}, \mathrm{TiF}$, and $\mathrm{TiF}_{2}$, are among other possible carriers of Ti in envelopes of O-rich stars (cf. Tsuji 1973). In M-type AGB stars and in TE, their abundances are expected to be orders of magnitude less than $\mathrm{TiO}$ and observations of their spectral features were considered to be very unlikely. In particular, TiH is not expected to be present under TE conditions in the low-pressure environments of AGB stars, but non-equilibrium chemistry might produce traceable quantities. In Paper I, we reported a tentative discovery of electronic absorption bands of $\mathrm{AlH}$, which is a molecule that is also not expected in TE, implying that $\mathrm{TiH}$ is more likely. Also, TiH is a non-negligible carrier of $\mathrm{Ti}$ in dwarf stars of spectral types $M$ and later, especially those with low metallicity (Burrows et al. 2005). Electronic bands of TiS were observed in a near-infrared spectrum of one S-type AGB star (Jonsson et al. 1992). Although they are not expected to be important for dust formation, we included these species for completeness.

Titanium has five stable isotopes, ${ }^{46,47,48,49,50} \mathrm{Ti}$, whose solar and terrestrial relative abundance ratios are 11.2:10.1: 100.0:7.3:7.0, respectively (Lodders 2003; Berglund \& Wieser 2011). Most spectroscopic studies to date have focused on the dominant isotope, ${ }^{48} \mathrm{TiO}$ (hereafter simply TiO). Deriving observational constraints on the abundance of the rare isotopologues based on electronic bands is very challenging (Lambert \& Luck 1977) and the only attempt to derive them in Mira resulted in upper limits that are generally consistent with the terrestrial/solar values (Wyckoff \& Wehinger 1972). A tentative observation of an enhanced abundance of ${ }^{50} \mathrm{TiO}$ was reported for Mira in Lambert \& Luck (1977) but has never been confirmed. Such an enhancement is virtually possible in AGB stars through the slow-neutron capture process ( $s$-process) and the third dredgeup (e.g., Wasserburg et al. 2015). The $s$-process is expected to enhance the amounts of the rare isotopes relative to ${ }^{48} \mathrm{Ti}$ resulting in a very modest overabundance of ${ }^{46} \mathrm{Ti}$, considerable overabundance of ${ }^{47} \mathrm{Ti}$ and ${ }^{49} \mathrm{Ti}$, and largest increase of ${ }^{50} \mathrm{Ti}$ (Clayton 2003; Howard et al. 1972; Wasserburg et al. 2015). Considering that even in unprocessed matter the rare isotopes add up to $\sim 24 \%$ of the total titanium content, they are important tracers of Tibearing species and therefore are included in our study as well. In Sect. 5.1, we describe the spectroscopic information required to identify these species at the submm wavelengths.

\section{Observations}

In many respects, Mira is considered to be the prototypical M-type AGB star. Its mass-loss rate of $2 \times 10^{-7} M_{\odot} / \mathrm{yr}$ is representative of the whole class. With a pulsation period of $\sim 330$ days it is also representative of long period variables. Its temperature changes within the range of about $2900-3200 \mathrm{~K}$ and its bolometric luminosity is of about $9000 L_{\odot}$ (see Paper I and references therein). Mira was chosen as the target of our study owing to these prototypical properties and because it was relatively easy to collect rich observational material spanning from optical to millimeter wavelengths for Mira.

\subsection{Submillimeter/millimeter and far-infrared data}

Most of the mm-far-infrared observations used in our analysis have been presented in detail in Paper I. An extensive search for Ti-bearing species was performed on all available data from Atacama Pathfinder Telescope (APEX), Atacama Large submillimeter/Millimeter Array (ALMA), and Herschel Space Observatory (Herschel). Below we briefly describe only the most crucial datasets.

- Observations carried out with APEX in 2013-2015 utilized the $\mathrm{FLASH}^{+}$(Klein et al. 2014) and SHeFi-1 (Vassilev et al. 2008) receivers to cover multiple spectral ranges between 222 and $500 \mathrm{GHz}$ (see Table A.1 in Paper I). Several spectral setups were arranged to target lines of $\mathrm{TiO}$ and resulted in serendipitous discovery of $\mathrm{TiO}_{2}$ features. The observations were obtained with telescope beams of $13^{\prime \prime}-28^{\prime \prime}$ full width at half maximum (FWHM).

- One APEX spectral setup, centered on $222.7 \mathrm{GHz}$ and first observed in December 2013, was reobserved on 1, 5, and 7 August 2016, i.e., after the publication of Paper I. The combined data of 2013 and 2016 resulted in a nominal rms noise level of $1.1 \mathrm{mK}\left(T_{A}^{*}\right)$ per $3.8 \mathrm{MHz}$. The total observation time on source was $7.5 \mathrm{~h}$. Those observations were designed to detect two $J=7-6$ lines of TiO.

- All archival spectra obtained with the HIFI instrument (Roelfsema et al. 2012) on Herschel were examined, including those of the HIFISTARS key program (PI: V. Bujarrabal) and performance verification data. The spectra cover narrow frequency ranges between about $556.6 \mathrm{GHz}$ and $1902.4 \mathrm{GHz}$, however, spectra at the longest wavelengths were obtained with a very low sensitivity. The telescope beam is in the range $20^{\prime \prime}-38^{\prime \prime}$ FWHM for the part of data used here.

- Mira was observed by ALMA in Band 7 on four different observing runs, which are summarized in Table 1. The ALMA data obtained on 21 July 2015 are our primary source of information. The complex gains of those interferometric observations were (self-)calibrated on the strong continuum sources of the Mira A/B binary system. This procedure resulted in a nominal sensitivity of about $1.7 \mathrm{mJy} /$ beam per $0.97 \mathrm{~km} \mathrm{~s}^{-1}$, making them the most sensitive submm observations of Mira to date.

- We found $\mathrm{TiO}$ and $\mathrm{TiO}_{2}$ features in a Mira spectrum obtained with ALMA in Band 9. Those observations are described in detail in Planesas et al. (2016) (see also Table 1).

In Paper I, we used ALMA Band 6 data obtained within the Long Baseline Science Verification Campaign (Fomalont et al. 2015). Their angular resolution of $\sim 30$ mas is the highest among all $\mathrm{mm} / \mathrm{submm}$ data available for this source. The data do not cover any $\mathrm{TiO}$ or $\mathrm{TiO}_{2}$ features at a sufficiently high signal-to-noise ratio $(\mathrm{S} / \mathrm{N})$ to be analyzed here. Our best angular resolution of $\sim 145$ mas was achieved in the Band 7 data from 2015 in which the stellar photosphere is not resolved. Many features of interest, however, are observed at a very high $\mathrm{S} / \mathrm{N}$ allowing size measurements even for regions smaller than the beam size.

Interferometric data were processed and imaged in CASA 4.6 (McMullin et al. 2007) in the same way as described in Paper I. In particular, all spectral data were processed in CLEAN after continuum subtraction in the $u v$ domain (cf. Appendix B of Paper I). 
Table 1. ALMA observations used in this work.

\begin{tabular}{|c|c|c|c|c|c|}
\hline $\begin{array}{l}\text { Date } \\
\text { obs. }\end{array}$ & PI & $\begin{array}{c}\text { Frequency ranges } \\
(\mathrm{GHz})\end{array}$ & $\begin{array}{l}\text { Resolution } \\
\left(\mathrm{km} \mathrm{s}^{-1}\right)\end{array}$ & $\begin{array}{l}\text { Angular } \\
\text { resolution }\end{array}$ & Reference \\
\hline 24 Feb. 2014 & Ramstedt & $330.2-334.0,342.3-346.1$ & 0.4 & $1 " .6 \times 0 \times 0^{\prime \prime} 5$ & Ramstedt et al. (2014) \\
\hline 03 May 2014 & Ramstedt & $330.2-334.0,342.3-346.1$ & 0.4 & $0^{\prime \prime} .5 \times 0, .4$ & Ramstedt et al. (2014) \\
\hline \multirow[t]{2}{*}{ 12-15 Jun. 2014} & Planesas & $330.4-330.7,345.6-345.9$ & 0.1 & $0 \prime 3 \times 0,3$ & Planesas et al. (2016) \\
\hline & & $331.1-332.8,343.7-345.5$ & 13.6 & & \\
\hline 21 Jul. 2015 & Kamiński & $342.2-345.9,354.2-357.9$ & 0.9 & $0 \prime \prime 16 \times 0 \prime \prime 13$ & Paper I \\
\hline 16 Jun. 2014 & Planesas & $679.11-682.77$ & 6.9 & $0^{\prime \prime} .23 \times 0, .16$ & Planesas et al. (2016) \\
\hline
\end{tabular}

\subsection{Optical spectroscopy}

We use the optical spectra presented in PaperI. These are 30 high-resolution $(R>5000)$ observations extracted from archives and covering the time span from December 1965 to August 2012. They come from a dozen of different observatories and instruments. We supplement these Paper I data with spectra obtained at the Mercator telescope and kindly provided to us by $\mathrm{H}$. van Winckel. All spectra were acquired with the high-resolution mode (HRF) of the HERMES instrument (Raskin et al. 2011) resulting in the coverage 377-900 $\mathrm{nm}$ at $R \sim 85000$. Twelve epochs were covered between July 2009 and August 2014, but most of the data come from 2009. In Table A.1, we list the observation dates and the corresponding visual phases and visual magnitudes calculated in the same way as in Paper I. The exposure times were in the range 6-900 s which, combined with different variability phases, resulted in a very broad range of $\mathrm{S} / \mathrm{N}$ ratios. All the HERMES spectra were reduced with the default pipeline.

Most of the collected spectra are not calibrated in flux. The spectra spatially correspond to a region of the size of the astronomical seeing on the given night but unfortunately the information about seeing is not available for most of the data. Even with those shortcomings, all the optical spectra that we collected for Mira constitute the richest and longest spanning spectroscopic record of variability in a Mira star to date.

\section{The state and structure of Mira's atmosphere}

The main source of information on titanium oxides here is the dataset obtained with ALMA in 2015 at visual phase 0.21, i.e., close to the highest bolometric brightness, which occurs at $\varphi \approx 0.17$. At approximately this phase, the pulsation shock arises from below the photosphere and the star appears hottest. Below we characterize different parts of Mira's envelope at this epoch. As in Paper I, we define $R_{\star}=14.4$ mas $\left(2.4 \times 10^{13} \mathrm{~cm}=331 R_{\odot}\right)$ as an idealized typical optical/infrared stellar radius, which we use here as a characteristic scale that is independent of the pulsation phase. Also following definitions introduced in Paper I (Sect. 1.2), we refer here to the extended atmosphere as the region with a radius of $3-4 R_{\star}$, where the pulsation shock strongly influences the kinematics of circumstellar gas. The dust formation zone, where the bulk of dust mass is built up from silicates and where the wind acceleration takes place, is above this region. At about $10 R_{\star}$ the material is accelerated to the terminal velocity and we treat this region as the inner boundary of the stellar wind.

\subsection{Continuum}

The continuum emission of Mira A at submm wavelengths is dominated by the radio photosphere (Reid \& Menten 1997, 2007). We measured the submm continuum source by fitting models to calibrated visibilities. A Gaussian fit resulted in a FWHM of $33.3 \times 32.8( \pm 0.3)$ mas and a corresponding photospheric temperature of $4805 \mathrm{~K}$. However, a uniform disk is thought to better represent the radio photosphere (Vlemmings et al. 2015; Matthews et al. 2015; Wong et al. 2016). Our data can be reproduced by an elliptical disk with a full size of $53.8 \times 49.5( \pm 0.1)$ mas at $\mathrm{PA}=-54.7^{\circ} \pm 0.6^{\circ}$, which corresponds to a brightness temperature of $2440 \mathrm{~K}$. This disk size and temperature are consistent, within $15 \%$, with those measured in long-baseline Band 6 data and correspond to phases 0.4-0.5 (Wong et al. 2016). The large discrepancy in the derived brightness temperature, 4805 versus $2440 \mathrm{~K}$, is mainly due to systematic errors and the unknown brightness distribution of the source. We assume here that a blackbody source of $2440 \mathrm{~K}$ with a radius of $\langle r\rangle=25.8$ mas $=1.8 R_{\star}$ underlies the circumstellar gas during the 2015 observations.

\subsection{Gas in the extended atmosphere}

To gain a better understanding of the structure of the atmosphere and envelope of Mira during this phase, we investigated spectral features from molecules other than titanium oxides. The ALMA spectra cover over 140 transitions of following species (and their isotopologues): $\mathrm{CO} v=0$ and 1; $\mathrm{SiO} v=0,1,2$, and 3; $\mathrm{SO} v=0$ and $1 ; \mathrm{SO}_{2} v=0$ and $v_{2}=1 ; \mathrm{AlO} ; \mathrm{SiS} ; \mathrm{HCN} v=0$ and $v_{2}=1$; and $\mathrm{H}_{2} \mathrm{O} v=0$ and $v_{1}=1$. About a third of the features remain unidentified but it is out of the scope of this paper to attempt a full identification. The observed transitions cover a wide range of upper energy levels $\left(E_{\mathrm{u}}\right)$, from a few tens to thousands of $\mathrm{K}$, probing parts of Mira's envelope with drastically different temperatures and densities. Example line profiles for species present in the innermost envelope are shown in Fig. 1.

Gas of low excitation is traced by pure emission lines that are spatially extended and centered at the stellar center-ofmass velocity, $V_{\mathrm{LSR}}=46.8 \mathrm{~km} \mathrm{~s}^{-1}$. Generally, emission from gas with a temperature lower than $100 \mathrm{~K}$ appears extended for our 145 mas beam. The maps of emission arising in this lowexcitation gas show the extended and complex wind that has already been described in detail (Ramstedt et al. 2014; Wong et al. 2016; Nhung et al. 2016). We are more interested here in the inner regions of the envelope where the dust forms. These regions are traced by lines of moderate and high excitation, which generally exhibit emission profiles centered at the stellar center-ofmass velocity (but see below). Additionally, low-excitation lines of very abundant species, such as $\mathrm{CO} v=0 J=3-2$ with an upper energy level of $33 \mathrm{~K}$ and lines of $\mathrm{SO}_{2}$ of moderate excitation (Müller \& Brünken 2005), also trace these regions as they display inverse P-Cyg profiles indicative of material infalling onto the star (Fig. 1, middle and right panels). The projected infall velocities are as high as $17 \mathrm{~km} \mathrm{~s}^{-1}$. While the emission components of the lines with low $E_{\mathrm{u}}$ arise in a very extended envelope, the 


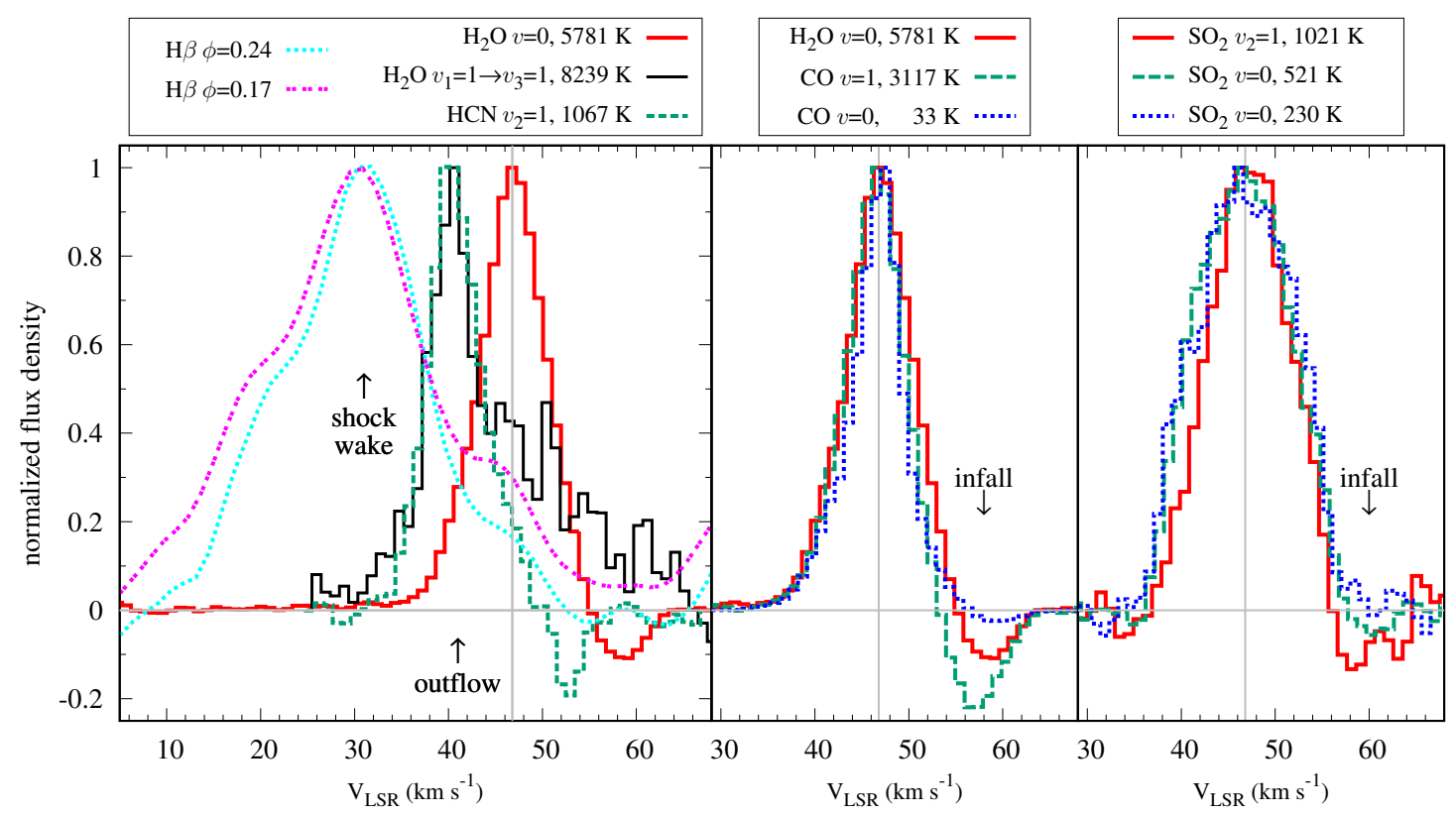

Fig. 1. Sample spectral profiles of transitions probing the atmosphere of Mira. The value of $E_{\mathrm{u}}$ is indicated for each transition in the legend. The transitions were chosen to show features requiring a broad range of excitation temperatures. The center-of-mass velocity of Mira is shown with the vertical line. The panels cover different velocity ranges but are in scale. All molecular spectra were extracted within an aperture of about $0^{\prime \prime} .2$ radius, which almost fully encompasses the emission of $\mathrm{TiO}$ and $\mathrm{TiO}_{2}$. Left: high-excitation lines, $\mathrm{H}_{2} \mathrm{O} v=017_{4,13} \rightarrow 16_{7,10}, \mathrm{H}_{2} \mathrm{O} v_{1}=1 \rightarrow v_{3}=1$ $12_{3,9} \rightarrow 11_{5,6}$, and $\mathrm{HCN} v_{2}=1 J=4 \rightarrow 3$. The two latter lines are seen at a peculiar radial velocity of $\sim 40 \mathrm{~km} \mathrm{~s}^{-1}$ and probe a region of the atmosphere that is affected by a shock. Also shown in this panel are two profiles of the $\mathrm{H} \beta$ hydrogen recombination lines observed in 2010 at phases 0.17 and 0.24 bracketing the phase of the ALMA data, $\varphi=0.21$. The hydrogen lines probe the recombining wake of the strong shock. Their profiles are slightly affected by absorption in photospheric lines of $\mathrm{TiO}$ and their broad profile is a result of scattering. Center: transition from levels in a broad range of $E_{\mathrm{u}}$ that show an absorption feature indicative of an infall. Right: lines of $\mathrm{SO}_{2}$ of moderate and high excitation. The $\mathrm{SO}_{2}$ profiles are wider (more parabolic) than any other molecular features observed.

absorption must be produced very close to the star where the gas is densest and has a very high opacity (cf. Schoenberg 1985).

Absorption and infall in high-excitation gas. The infalling part of the envelope is also seen in absorption in transitions requiring high-excitation temperatures, for instance, in CO $v=1 J=3-2$ $\left(E_{\mathrm{u}}=3117 \mathrm{~K}\right.$; Gendriesch et al. 2009) and $\mathrm{H}_{2} \mathrm{O} v=017_{4,13} \rightarrow$ $16_{7,10}\left(E_{\mathrm{u}}=5781 \mathrm{~K}\right.$, Yu et al. 2012). To produce absorption, this infalling gas has to have a temperature that is lower than the background continuum source. Our measured sizes of the lineabsorbing regions from various species are smaller than or comparable to the continuum source, which agrees with our interpretation that the absorption originates from gas seen against the stellar disk. The physical radial extent of the gas cannot be directly measured at the angular resolution of our data. However, absorption in the two lines is only possible in a region where the rotational energy levels above a few thousand $\mathrm{K}$ are effectively populated. The radial extent of these regions is roughly the same as the angular size of the corresponding emission components. The beam-deconvolved sizes of $93 \times 77( \pm 3)$ mas of the $\mathrm{CO}$ emission and $82 \times 71( \pm 2)$ mas for $\mathrm{H}_{2} \mathrm{O}$ set the upper limits on the size of the region dominated by infall motions. They correspond to a radius of about $5.5 R_{\star}$.

Emission from the extended atmosphere. When a region of about 0 ".2 radius is analyzed ${ }^{1}$, most of the emission features arise from the extended atmosphere. The emission profiles show a

\footnotetext{
1 This size corresponds to the entire extended atmosphere and encompasses the emission regions of titanium oxides (Sect. 3.1).
}

variety of shapes. For instance, the relative strengths of emission and absorption components produce slightly different net emission profiles, as illustrated in the middle panel of Fig. 1. Some differences are related to the intrinsic difference in abundance distribution. This is most apparent in profiles of $\mathrm{SO}_{2}$ (right panel of Fig. 1), which are broader than all other molecular lines observed.

Recombining shock-wake. Our optical spectra allow us to locate the shocked material. We examined the optical spectra obtained in 2010 for phases 0.17 and 0.24 and presented in Paper I (Appendix C; see also Fabas et al. 2011). These spectra are the closest in phase to the ALMA observations $(\varphi=0.21)$. They cover the $\mathrm{H} \beta$ Balmer line $\left(\lambda_{\text {lab }}=4861.35 \AA\right)$ blueshifted by $16.0 \mathrm{~km} \mathrm{~s}^{-1}$, i.e., to $V_{\mathrm{LSR}}=30.8( \pm 1.0) \mathrm{km} \mathrm{s}^{-1}$. The recombination lines trace the material cooling after the passage of the supersonic pulsation shock and of a temperature on the order of $10^{4} \mathrm{~K}$ (Fox et al. 1984). The lines are shown in Fig. 1 (left panel). We do not find any molecular species in the ALMA data that would correspond to this velocity. This is not surprising as molecules are thought to be effectively dissociated by the shock (Cherchneff 2006; Gobrecht et al. 2016). The recombination lines at phases close to 0.2 are a signature of the most recent pulsation shock that just left the optical/infrared photosphere. Because the shock emerges from below the optical photosphere at a visual phase of about -0.2 (e.g., Hinkle et al. 1984; Richter \& Wood 2001), since then it has had enough time to travel to a radius of $\sim 2 R_{\star}$ at the epoch of ALMA observations $(\varphi=0.2)$ and therefore was above the submm radio photosphere $\left(\langle r\rangle=1.8 R_{\star}\right)$. 
Shock signatures in molecular gas. Although molecules are absent in the recombining shock wake, our data show that molecular gas is affected by the shock. We identified transitions of $\mathrm{H}_{2} \mathrm{O}$ and $\mathrm{HCN}$ that require excitation of their vibrational modes (Yu et al. 2012; Thorwirth et al. 2003) and whose emission is centered at a peculiar radial velocity $V_{\mathrm{LSR}} \approx 40.3 \mathrm{~km} \mathrm{~s}^{-1}$, i.e., blueshifted by $6.5 \mathrm{~km} \mathrm{~s}^{-1}$ with respect to the stellar velocity. This velocity is exactly the same as that of rovibrational $\Delta v=3$ absorption lines of $\mathrm{CO}$ at the same pulsation phase of Mira (Hinkle et al. 1984). These absorption lines at $1.6 \mu \mathrm{m}$ require an excitation temperature of $\sim 3700 \mathrm{~K}$ at $\varphi=0.2$ and are thought to represent molecular material cooling after the passage of the shock in the deepest observable layers of the star (Hinkle 1978; Hinkle et al. 1984). The lines we observed with ALMA at this velocity are seen in emission and likely represent even hotter $(>3700 \mathrm{~K})$ gas closer to the active shock. Still, the temperatures must be below those at which $\mathrm{H}_{2} \mathrm{O}$ and $\mathrm{HCN}$ are effectively dissociated and characteristic to the recombination wake. The two strongest of those lines, para- $\mathrm{H}_{2} \mathrm{O} v_{1}=1 \rightarrow v_{3}=1 J_{K a, K c}=$ $12_{3,9} \rightarrow 11_{5,6}$ with $E_{\mathrm{u}}=8239 \mathrm{~K}$ and $\mathrm{HCN} v_{2}=1 \mathrm{~J}=4 \rightarrow 3$ with $E_{\mathrm{u}}=1067 \mathrm{~K}$, are shown in Fig. 1 . The former line is a rovibrational transition ${ }^{2}$ and has the highest $E_{\mathrm{u}}$ among all lines that we were able to identify in the ALMA spectrum. We interpret the emission at the peculiar velocity as arising in gas located in the expanding part of the atmosphere and excited directly by the new shock, likely in the post-shock layer where molecules start to reform (cf. Gobrecht et al. 2016). The small size of the region of the $\mathrm{H}_{2} \mathrm{O} v_{1}=1 \rightarrow v_{3}=1$ emission, $(89 \pm 11) \times(43 \pm 18)$ mas, and its location at exactly the continuum center strongly support our interpretation. This is the first time the direct pulsation-shock signatures have been identified in submm observations of a Mira. It is also the first strong observational evidence that carbonbearing species, such as $\mathrm{HCN}$, are formed by shock chemistry in M-type stars, as advocated in multiple theoretical studies (e.g., Duari et al. 1999; Cherchneff 2006; Gobrecht et al. 2016).

For our further discussions it is sufficient that we identified the shock-excited molecular region and defined its kinematical location. We do not aim to explain the complex shock phenomena in Mira's atmosphere in this paper. In the next sections, we associate the signatures of titanium-bearing species with the different molecular regions that we defined above.

\section{Submillimeter lines of titanium oxides}

\subsection{Line lists}

In order to investigate the presence of the isotopologues of the titanium oxides at $\mathrm{mm}$ to far-infrared wavelengths, we created line lists based on spectroscopic data from different sources.

Titanium monoxide is a radical with two unpaired electrons with a regular ${ }^{3} \Delta$ ground electronic state, thus $\Omega=1$ is the lowest spin component and $\Omega=3$ is the highest spin component. TiO has a large dipole moment of $3.34 \pm 0.01 \mathrm{D}$ in its ground electronic state (Steimle \& Virgo 2003). Predictions of the rotational spectrum of ${ }^{48} \mathrm{TiO}$ were taken from the Cologne Database for Molecular Spectroscopy (CDMS; Müller et al. 2001, 2005). The entry is based on the report by Namiki et al. (1998). We prepared entries of the minor isotopic species based

\footnotetext{
2 Its spectroscopic line strength originates from the vibration-rotation interaction of the $v_{1}=1$ and $v_{3}=1$ states. Throughout this paper, we use the quantum number convention where the direction of the arrow indicates emission, i.e., a transition from the upper to the lower level. When no vibrational quantum number is given, the transition is within the ground vibrational state.
}

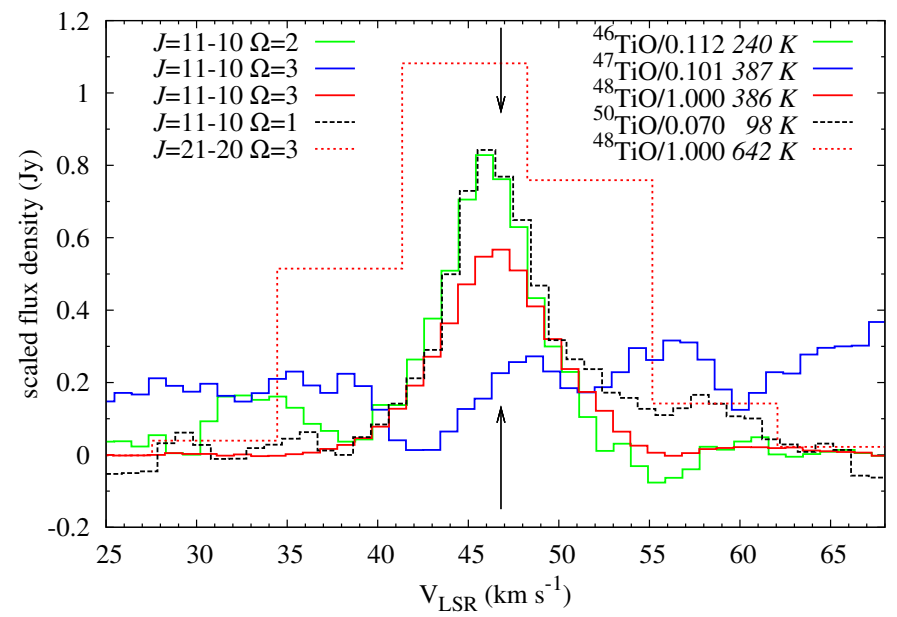

Fig. 2. Line profiles of $\mathrm{TiO}$ isotopologues observed with ALMA (cf. Table 2). One line of ${ }^{50} \mathrm{TiO}$ observed in June 2014 is not shown because is covered by one resolution element only. The flux densities were scaled by their relative solar isotopic ratios, as indicated in the right legend. The arrows indicate the center-of-mass velocity of Mira. Spectra were extracted from regions encompassing the entire emission region.

on measurements of ${ }^{46} \mathrm{TiO},{ }^{48} \mathrm{TiO}$, and ${ }^{50} \mathrm{TiO}$ between 250 and $345 \mathrm{GHz}$ of Kania et al. (2008) and unpublished measurements (Kania, in prep.).

Titanium dioxide is a bent molecule with a $\tilde{X}^{1} A_{1}$ electronic ground state and a large dipole moment of $6.33 \pm 0.07 \mathrm{D}$ (Wang et al. 2009). Predictions of the rotational spectra of ${ }^{48} \mathrm{TiO}_{2},{ }^{46} \mathrm{TiO}_{2}$, and ${ }^{50} \mathrm{TiO}_{2}$ were taken from the CDMS. The entries are based on the mm-wave measurements and analysis of Kania et al. (2011), which included additional data of these isotopologues from Fourier transform microwave (FTMW) measurements by Brünken et al. (2008). Predictions for ${ }^{47} \mathrm{TiO}_{2}$ and ${ }^{49} \mathrm{TiO}_{2}$ were produced based on FTMW measurements of additional isotopologues from Müller et al. (2007) and unpublished measurements (Kania, in prep.).

In addition to the rotational lines of the oxides and their rare isotopologues, it is interesting to search for signatures of other Ti-bearing species mentioned in Sect. 2. Accurate frequencies of rotational transitions are known for TiN, TiS, and TiF (Namiki et al. 1998; Pulliam et al. 2010; Sheridan et al. 2003) or can be predicted, as for $\mathrm{TiH}$, with the available spectroscopic constants. However, our sensitive observations did not cover strong transitions of any of these species.

\subsection{Identification of $\mathrm{TiO}, \mathrm{TiO}_{2}$, and their isotopologues}

Prior to this work, pure rotational lines of $\mathrm{TiO}$ and $\mathrm{TiO}_{2}$ have only been known in the mm-wave spectrum of the red supergiant VY CMa (Kamiński et al. 2013; De Beck et al. 2015). Because Mira is only the second known source with those circumstellar molecules, we describe the identification of the $\mathrm{mm} / \mathrm{submm}$ features of $\mathrm{TiO}$ and $\mathrm{TiO}_{2}$ in detail.

\subsubsection{Identification of $\mathrm{TiO}$}

A list of all the TiO transitions covered by APEX, ALMA, and Herschel, dates of observations, noise levels, and fluxes are given in Table 2. In all, we detected five different transitions of ${ }^{48} \mathrm{TiO}$.

In the APEX spectrum of Mira from 2013, we serendipitously detected a feature at $420.224 \mathrm{GHz}$ for which the most 
T. Kamiński et al.: Ti-bearing gas and dust in Mira

Table 2. Transitions of TiO covered by APEX, Herschel, and ALMA.

\begin{tabular}{|c|c|c|c|c|c|c|c|c|}
\hline $\begin{array}{c}\text { Isoto- } \\
\text { pologue }\end{array}$ & $\begin{array}{l}J_{\text {up }} \rightarrow J_{\text {low }} \\
\text { multiplet } \Delta_{\Omega}\end{array}$ & $\begin{array}{c}v \\
(\mathrm{MHz})\end{array}$ & $\begin{array}{l}\operatorname{Err}(v)^{a} \\
\left(\mathrm{~km} \mathrm{~s}^{-1}\right)\end{array}$ & $\begin{array}{c}S \mu^{2} \\
\left(\text { Debye }^{2}\right) \\
\end{array}$ & $\begin{array}{c}E_{\mathrm{u}} \\
(\mathrm{K})\end{array}$ & $\begin{array}{c}\text { Dates of } \\
\text { observation }\end{array}$ & $\begin{array}{l}\text { Vis. } \\
\text { phase }\end{array}$ & $\begin{array}{l}\text { Flux }(1 \sigma) \\
\left(\mathrm{Jy} \mathrm{km} \mathrm{s}^{-1}\right)\end{array}$ \\
\hline \multicolumn{9}{|c|}{ APEX } \\
\hline \multirow[t]{5}{*}{${ }^{48} \mathrm{TiO}$} & $7 \rightarrow 6, \Delta_{1}$ & 221580.45 & 0.05 & 153.0 & 41.0 & 3-20 Dec. 2013, 1-7 Aug. 2016 & $0.5 / 0.35$ & $0.89(0.21)$ \\
\hline & $7 \rightarrow 6, \Delta_{2}$ & 224138.74 & 0.03 & 143.4 & 180.7 & 3-20 Dec. $2013,1-7$ Aug. 2016 & $0.5 / 0.35$ & $<0.60$ \\
\hline & $11 \rightarrow 10, \Delta_{3}$ & 355623.35 & 0.04 & 227.2 & 386.2 & 30 Jun.-6 Jul. 2014 & 0.0 & $3.14(0.73)$ \\
\hline & $13 \rightarrow 12, \Delta_{3}$ & 420224.14 & 0.04 & 274.7 & 425.0 & 13-14 Aug. 2013 & 0.1 & $9.67(1.81)$ \\
\hline & $15 \rightarrow 14, \Delta_{3}$ & 484795.99 & 0.06 & 321.4 & 470.0 & 1-8 Jul. 2014 & 0.0 & $8.66(1.50)$ \\
\hline \multicolumn{9}{|c|}{ Herschel } \\
\hline \multirow[t]{6}{*}{${ }^{48} \mathrm{TiO}$} & $18 \rightarrow 17, \Delta_{1}$ & 569537.72 & 0.13 & 400.3 & 258.2 & 19 Jul. 2010 & 0.7 & $<22.57$ \\
\hline & $18 \rightarrow 17, \Delta_{2}$ & 575979.23 & 0.13 & 396.5 & 400.4 & 19 Feb. 2010 & 0.3 & $<21.97$ \\
\hline & $20 \rightarrow 19, \Delta_{3}$ & 646070.05 & 0.26 & 436.2 & 609.6 & 20 Jul. 2010 & 0.7 & $<28.20$ \\
\hline & $25 \rightarrow 24, \Delta_{3}$ & 807067.03 & 0.75 & 549.9 & 787.7 & 4 Feb. 2010 & 0.2 & $<30.80$ \\
\hline & $29 \rightarrow 28, \Delta_{3}$ & 935619.31 & 1.48 & 640.2 & 958.1 & 10 Aug. 2011, 28 Jan. $2012^{b}$ & $0.9 / 0.4$ & $<222.89$ \\
\hline & $34 \rightarrow 33, \Delta_{3}$ & 1095946.06 & 2.98 & 752.8 & 1205.7 & 20 Jul. 2010 & 0.7 & $65.25(18.31)$ \\
\hline \multicolumn{9}{|c|}{ ALMA } \\
\hline${ }^{50} \mathrm{TiO}$ & $11 \rightarrow 10, \Delta_{1}$ & 344722.34 & $>0.02$ & 240.9 & 97.8 & 12-15 Jun. 2014 & 1.0 & $0.31(0.02)$ \\
\hline${ }^{48} \mathrm{TiO}$ & $21 \rightarrow 20, \Delta_{3}$ & 678293.80 & 0.33 & 459.0 & 642.1 & 16 Jun. 2014 & 1.0 & $16.36(1.00)$ \\
\hline${ }^{46} \mathrm{TiO}$ & $11 \rightarrow 10, \Delta_{2}$ & 355975.66 & $>0.02$ & 239.8 & 240.2 & 21 Jul. 2015 & 0.2 & $0.54(0.02)$ \\
\hline${ }^{47} \mathrm{TiO}$ & $11 \rightarrow 10, \Delta_{3}$ & 357526.69 & $>0.04$ & 228.4 & 386.8 & 21 Jul. 2015 & 0.2 & $<0.33(0.02)$ \\
\hline${ }^{48} \mathrm{TiO}$ & $11 \rightarrow 10, \Delta_{3}$ & 355623.35 & 0.04 & 227.2 & 386.2 & 21 Jul. 2015 & 0.2 & $4.44(0.03)$ \\
\hline${ }^{50} \mathrm{TiO}$ & $11 \rightarrow 10, \Delta_{1}$ & 344722.34 & $>0.02$ & 240.9 & 97.8 & 21 Jul. 2015 & 0.2 & $0.39(0.02)$ \\
\hline
\end{tabular}

Notes. Spectroscopic data for $e$ and $f$ parity components were combined. The flux is given with a $1 \sigma$ error in the parenthesis. Upper limits on flux have a $3 \sigma$ significance. ${ }^{(a)}$ The uncertainty of transition frequency expressed in velocity units; from CDMS. ${ }^{(b)}$ Mapping observations. The examined spectrum is an average from two dates and located at an offset of $5^{\prime \prime 2} 2$ (or 0.24 the beam width) from the actual stellar position.

likely identification is the $\Delta_{3} J=13-12$ line of TiO. As a verification of this identification, we next obtained a deep spectrum covering the $J=7 \rightarrow 6$ transition of $\Delta_{1}$ and $\Delta_{2}(221.580$ and $224.139 \mathrm{GHz}$ ) with APEX. Both lines were observed earlier in VY CMa. The Mira observations were obtained in December 2013, i.e., two months after the observations of the feature at $420.224 \mathrm{GHz}$. The deep integration resulted in a nondetection of the anticipated $\mathrm{TiO} J=7 \rightarrow 6$ lines. Later in 2014, we obtained APEX observations covering two more lines, $\Delta_{3} J=11 \rightarrow 10$ (at $355.623 \mathrm{GHz}$ ) and $\Delta_{3} J=15 \rightarrow 14$ (at $484.796 \mathrm{GHz}$ ), which have $E_{\mathrm{u}}$ values close to the $\Delta_{3} J=13 \rightarrow 12$ line at 420.2 GHz. Both were detected. An example APEX spectrum of TiO is shown in Fig. 1 of Paper I. These discovery observations of $\mathrm{TiO}$ were later followed up with ALMA. Additionally, in 2016 we repeated observations of the two $J=7 \rightarrow 6$ lines with APEX and the $\Delta_{2}$ transition was detected at a $4.5 \sigma$ level in the combined 2013 and 2016 data. All the lines observed are very closely aligned in velocity and have a centroid position at $46.3 \pm 0.8 \mathrm{~km} \mathrm{~s}^{-1}$, which is consistent with the center-of-mass velocity of Mira.

The ALMA observations from 2015 were designed to observe the $\Delta_{3} J=11 \rightarrow 10$ line of TiO and three additional transitions of the ${ }^{46,47,50} \mathrm{TiO}$ isotopologues. The main reason to re-observe the ${ }^{48} \mathrm{TiO}$ line (earlier covered by APEX) was to measure the size of the emission. All targeted lines were detected except the $\Delta_{3} J=11 \rightarrow 10$ line of ${ }^{47} \mathrm{TiO}$. In addition, the ALMA observations in Bands 9 and 7 from June 2014 serendipitously covered one more transition of $\mathrm{TiO} \Delta_{3} J=21 \rightarrow 20$ and one of ${ }^{50} \mathrm{TiO} \Delta_{1} J=11 \rightarrow 10$, which are observed at a high $\mathrm{S} / \mathrm{N}$ but at a spectral resolution that is too poor to resolve the line profiles. The Band 9 spectrum also covered one transition of ${ }^{47} \mathrm{TiO}$, but it is buried in a strong line of $\mathrm{SO} v=1$. The ALMA observations from February and May 2014 also covered the $\Delta_{1} J=11 \rightarrow 10$ transition of ${ }^{50} \mathrm{TiO}$, but their combined sensitivity was not sufficient to detect the line. The spectrally resolved lines observed with ALMA are shown in Fig. 2.

In searching for $\mathrm{TiO}$ emission at far-infrared wavelengths, we examined all available Herschel/HIFI scans. We found spectra covering six transitions of TiO but only one seems to be detected with a low confidence. Rest frequencies of $\mathrm{TiO}$ lines above $\sim 600 \mathrm{GHz}$ are more uncertain than at lower frequencies (cf. Table 1, Col. 4), making the identification in Herschel spectra more challenging. Nevertheless, we found a feature at the expected position of the $34 \rightarrow 33, \Delta_{3}$ line whose flux is $3.5 \sigma$ above the local noise level (see Fig. 1 in Paper I), and we consider the line tentatively detected.

Two transitions, $11 \rightarrow 10, \Delta_{3}$ of $\mathrm{TiO}$ and $11 \rightarrow 10, \Delta_{1}$ of ${ }^{50} \mathrm{TiO}$, were observed at multiple epochs and their fluxes remained unchanged within the observational uncertainties. The $\mathrm{mm} / \mathrm{submm}$ data in hand do not indicate any substantial variability in the pure rotational lines of $\mathrm{TiO}$.

\subsubsection{Identification of $\mathrm{TiO}_{2}$ emission}

Titanium dioxide has many rotational transitions with comparable intensity at submm wavelengths. Each individual line is rather weak and a sensitivity higher than that for $\mathrm{TiO}$ is required to observe $\mathrm{TiO}_{2}$ emission (Kamiński et al. 2013). Nevertheless, we succeed in detecting the main isotopologue of $\mathrm{TiO}_{2}$ and some of the rarer isotopic species.

The spectra of Mira collected with APEX covered several transitions of $\mathrm{TiO}_{2}$. From these, only four lines are present at the expected frequencies, $J_{K_{a}, K_{c}}=19_{7,13} \rightarrow 19_{6,14}, 15_{7,9} \rightarrow 15_{6,10}$, $38_{1,37} \rightarrow 38_{0,38}$, and $31_{5,27} \rightarrow 30_{4,26}$. The emission features are of very modest $\mathrm{S} / \mathrm{N}$, in the range $3-5$. These lines are considered tentatively detected and, although not analyzed in detail here, were the stepping stone to observing $\mathrm{TiO}_{2}$ with ALMA. 

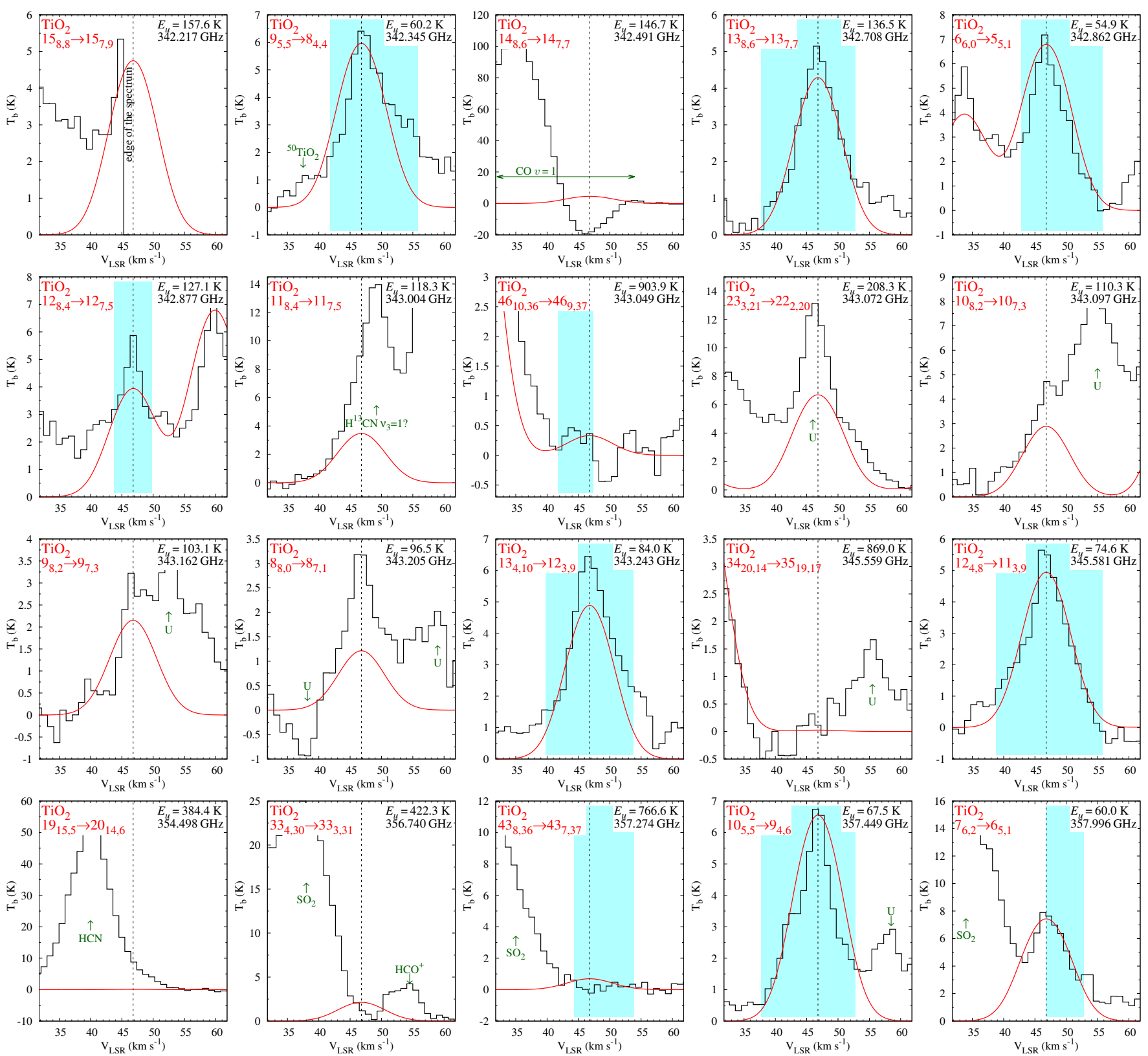

Fig. 3. All $\mathrm{TiO}_{2}$ transitions covered by the ALMA 2015 data. The black histograms present the observations and the red line shows a simulation based on the best-fit excitation model of $\mathrm{TiO}_{2}$ found with CASSIS. Each panel is centered on the transition labeled in the upper left corner and the central velocity is indicated with a vertical dashed line. When another line of $\mathrm{TiO}_{2}$ is covered by the plot, its simulation is also shown. The highlighted parts of the $\mathrm{TiO}_{2}$ profiles were used in our minimization routine. Many of the lines shown are contaminated by emission of other species, the strongest of which are labeled in green. Lines with $E_{\mathrm{u}}$ higher than about $300 \mathrm{~K}$ were not detected and their spectra are shown for completeness.

Our most sensitive ALMA data, obtained in 2015, covered 20 transitions of $\mathrm{TiO}_{2}$ with $E_{\mathrm{u}} \leq 904 \mathrm{~K}$. Five of these transitions are not strongly contaminated by emission of other lines and have a high $\mathrm{S} / \mathrm{N}$. Five other lines blend tightly with other stronger features so it is difficult to assess if they are present. Lines with $E_{\mathrm{u}}>300 \mathrm{~K}$ are not detected. The remaining lines are partially blended with other features. Although they are recognizable as $\mathrm{TiO}_{2}$ features, their fluxes cannot be directly measured. ALMA observations in Band 7 from earlier epochs (Table 1) were not sensitive enough to detect $\mathrm{TiO}_{2}$ emission. All the transitions covered are shown in Fig. 3. The figure labels provide basic information about the covered lines.

After excitation conditions of $\mathrm{TiO}_{2}$ gas were constrained using the ALMA Band 7 data from 2015 (Sect. 5.5), we examined the Band 9 data for the presence of $\mathrm{TiO}_{2}$. The identification was more challenging there because of the low spectral resolution and severe line blending. The strongest predicted emission feature is a close blend of two transitions of $\mathrm{TiO}_{2}, 13_{11,3} \rightarrow 12_{10,2}$ and $19_{9,11} \rightarrow 18_{8,10}$, near $675.9 \mathrm{GHz}$ and we found there an emission feature of the predicted intensity. Other lines are either too weak or are blended with much stronger emission features that have not yet been identified. Still, the observation of the $675.9 \mathrm{GHz}$ blend confirms our identification and excitation analysis.

Because the $\mathrm{S} / \mathrm{N}$ of the strongest $\mathrm{TiO}_{2}$ lines observed with ALMA is $\sim 93$, we searched for the rare isotopologues of $\mathrm{TiO}_{2}$, which are expected to have spectral features approximately ten times weaker than ${ }^{48} \mathrm{TiO}_{2}$. The search results are as follows. 

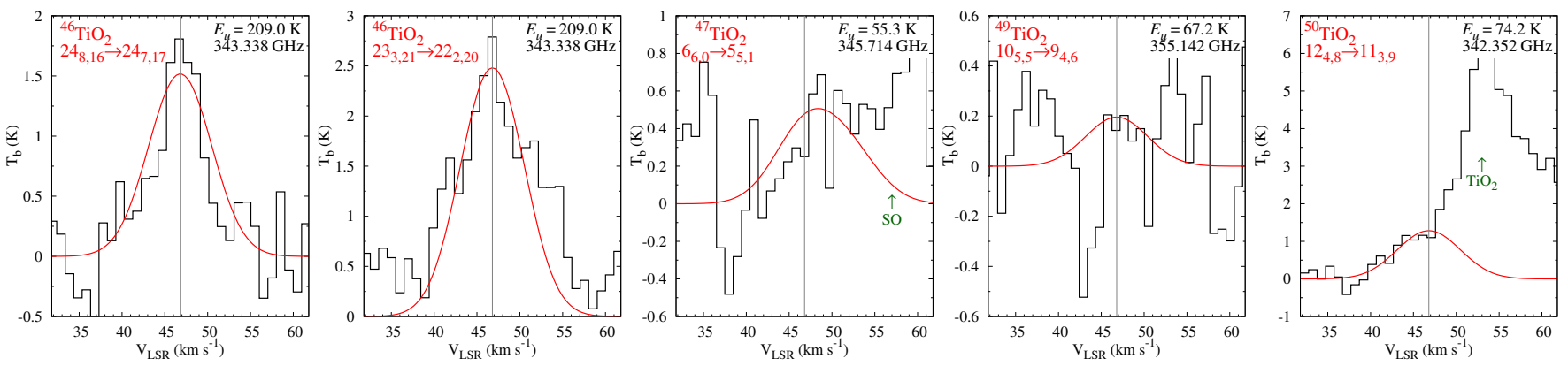

Fig. 4. Sample spectra of the rare isotopologues of $\mathrm{TiO}_{2}$. Black histograms show observations and the red lines show CASSIS simulations corresponding to upper limits on the column densities of the rare isotopologues. Only features of ${ }^{46} \mathrm{TiO}_{2}$ are considered detections.

- We found two unblended (out of 11 covered) lines of ${ }^{46} \mathrm{TiO}_{2}$ and their identification as the $24_{8,16} \rightarrow 24_{7,17}$ and $23_{3,21} \rightarrow$ $22_{2,20}$ transitions is firm (see Fig. 4).

- We covered 14 transitions of ${ }^{47} \mathrm{TiO}_{2}$. Three weak unidentified features were found close to the calculated positions of ${ }^{47} \mathrm{TiO}_{2}$, but the line centers are displaced by a few $\mathrm{km} \mathrm{s}^{-1}$ and none can be confidently identified as ${ }^{47} \mathrm{TiO}_{2}$.

- Nine transitions of ${ }^{49} \mathrm{TiO}_{2}$ were covered and one unblended feature was identified as possibly ${ }^{49} \mathrm{TiO}_{2}$, i.e., $76,2 \rightarrow 6_{5,1}$. Its $\mathrm{S} / \mathrm{N}$ is too low, however, to consider this identification as firm.

- A feature near $342.30 \mathrm{GHz}$ is blended with a line of ${ }^{48} \mathrm{TiO}_{2}$ (Fig. 3, first row, second column) and is most likely the $12_{4,8} \rightarrow 11_{3,9}$ transition of ${ }^{50} \mathrm{TiO}_{2}$. No other clean lines of this isotopologue are present in the spectrum.

Overall, we are only confident about the identification of the two lines of ${ }^{46} \mathrm{TiO}_{2}$. Sample spectra are shown in Fig. 4.

\subsection{Spatio-kinematical characteristics of TiO-bearing gas}

The ALMA observations of the $11 \rightarrow 10, \Delta_{3}$ transition have the best angular resolution $\left(0^{\prime \prime} .154 \times 00^{\prime \prime} .125\right)$ and $\mathrm{S} / \mathrm{N}(\sim 248)$ among all the data collected for $\mathrm{TiO}$ and its isotopologues. We base our analysis mainly on this single dataset. The line profile is shown in Fig. 5 and maps are shown in Figs. 6 and 7.

The line profile is dominated by emission centered at the stellar center-of-mass velocity and resembles profiles typical for species of moderate-to-high excitation and limited extent, typical of the extended atmosphere. At a closer inspection and for the central resolution element (central beam), however, a weak absorption component is seen at $56.5 \mathrm{~km} \mathrm{~s}^{-1}$ and the overall profile can be classified as an inverse P-Cyg type, as shown in Fig. 5. In addition, in the spectral range where the comparison profile of $\mathrm{H}_{2} \mathrm{O}$ in Fig. 5 is dominated by absorption, the TiO feature displays a weak emission component, at $57-67 \mathrm{~km} \mathrm{~s}^{-1}$.

The spatial distribution of the main emission component within the $35-56 \mathrm{~km} \mathrm{~s}^{-1}$ interval, is very well approximated by a Gaussian of a beam-deconvolved FWHM of $115.9 \times 93.3( \pm 2.7)$ mas and a position angle of the major axis (PA) of $106^{\circ} \pm 5^{\circ}$. This emission is much more extended than the submm photosphere. The centroid position is $9.3 \pm 0.2$ mas east and $16.3 \pm 0.4$ mas north from the continuum peak (Fig. 6), so the net location of $\mathrm{TiO}$ is not far from the stellar center. Most of the gas giving rise to $\mathrm{TiO}$ emission is therefore surrounding the star within the radius $(F W H M / 2)$ of about $4 R_{\star}$.

The $\mathrm{TiO}$ main emission region is only slightly larger than that of the $\operatorname{AlO} N=9-8$ line of $123 \times 74$ mas observed at the same angular resolution (Paper I). The spatial offset of the centroid with respect to the continuum center is 12 mas, which is

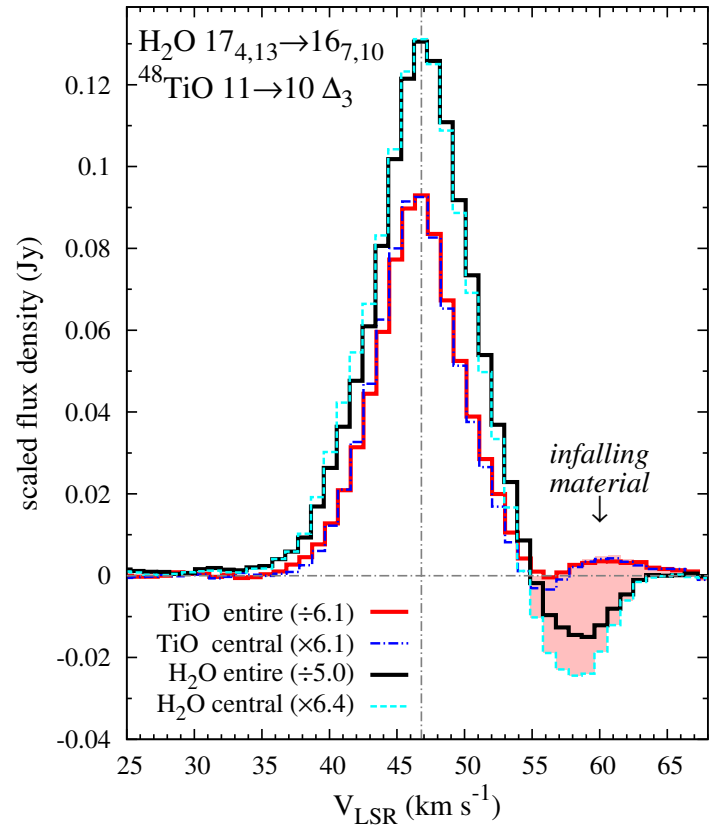

Fig. 5. Profiles of the TiO $11 \rightarrow 10, \Delta_{3}$ transition are compared to these of $\mathrm{H}_{2} \mathrm{O} v=017_{4,13} \rightarrow 16_{7,10}$. Spectra were extracted for the central beam and within the entire emission region. The flux densities were arbitrarily scaled by the listed factors for a more readable comparison. Shaded in red are signatures of infall in Mira.

much smaller than that for AlO (40 mas). On the basis of the map in which the $N=6-5$ emission of AlO is resolved well, the apparent centroid of $\mathrm{AlO}$ emission is displaced from the stellar disk as a consequence of a highly anisotropic distribution of $\mathrm{AlO}$ around the star. Although details of $\mathrm{TiO}$ distribution require observations at a higher angular resolution, our maps suggest that while the extent of $\mathrm{TiO}$ emission is similar to that of $\mathrm{AlO}$, the $\mathrm{TiO}$ emission is distributed much more isotropically around the star than AlO.

The absorption of $\mathrm{TiO}$ seen at $V_{\mathrm{LSR}}=56-57 \mathrm{~km} \mathrm{~s}^{-1}$ is spatially compact and is at the center of the continuum source. It is therefore a signature of the portion of TiO-bearing gas seen in the line of sight toward the stellar disk. To appear in absorption, the temperature of $\mathrm{TiO}$ gas must be lower than that of the photosphere, $<2440 \mathrm{~K}$. By comparing the emission-to-absorption intensity ratio in the profile of $\mathrm{TiO}$ to that of species requiring excitation temperatures on the order of $1000 \mathrm{~K}$, as in Fig. 5, one can see that although $\mathrm{TiO}$ is excited in the hot infall, the bulk of TiO-bearing gas must reside in gas characterized by a much lower temperature (see Sect. 5.5). 

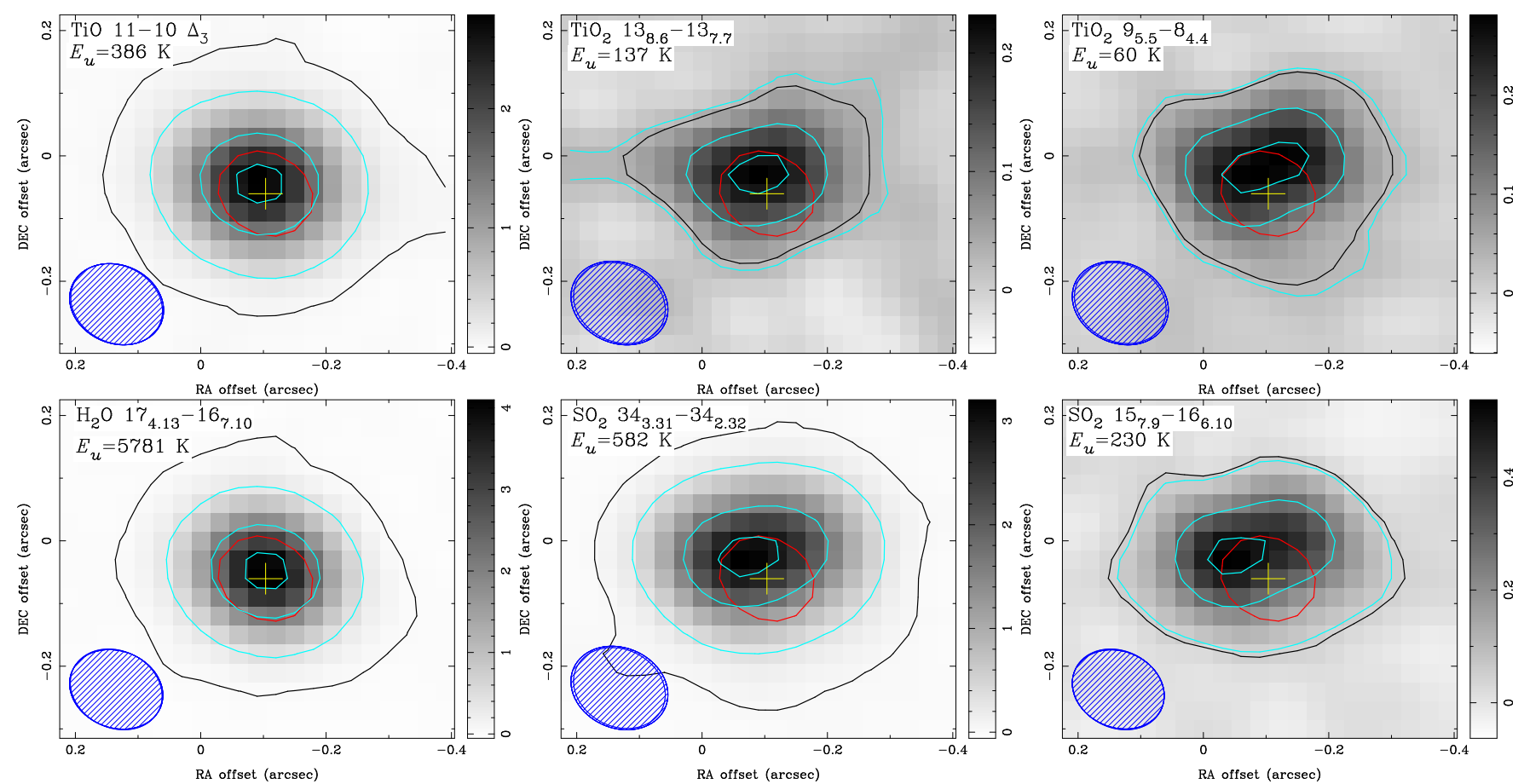

Fig. 6. Maps of molecular emission in the extended atmosphere of Mira. The emission was integrated in the spectral range corresponding to the main emission component between about 35 and $55 \mathrm{~km} \mathrm{~s}^{-1}$ (optimized for each transition). The emission is shown in gray in units of Jy km s ${ }^{-1}$ and with cyan contours at 10,50, and $90 \%$ of the maximum flux. Additionally, a black contour at the $3 \sigma$ noise level is shown. The location of continuum center of Mira A is indicated with a plus symbol whose size corresponds to the physical size of the stellar disk. The red contour shows the continuum emission at $50 \%$ of the peak value and represents the continuum extent smeared by the synthesized beam. The FWHMs of the beams are shown with hatched ellipses.
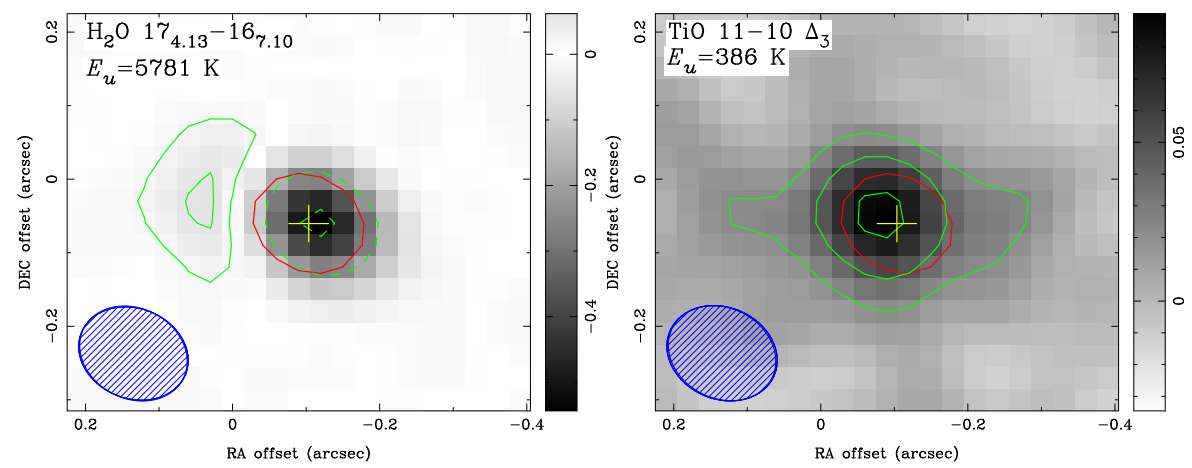

Fig. 7. Maps of the infall signatures. The maps were produced by integrating the flux density within $55-64 \mathrm{~km} \mathrm{~s}^{-1}$ for $\mathrm{H}_{2} \mathrm{O} 17_{4,13} \rightarrow 16_{7,10}$ and within $57-67 \mathrm{~km} \mathrm{~s}^{-1}$ for TiO $J=11 \rightarrow 10 \Delta_{3}$ (cf. Fig. 5). The water line at this range is dominated by absorption (negative flux), which is shown with green dashed contours, and residual emission outside the stellar disk (positive flux), which is shown with solid contours. The infall in the $\mathrm{TiO}$ line is seen mainly in emission (solid contours). The symbols have the same meaning as in Fig. 6.

The weak $\mathrm{TiO}$ emission in the $57-67 \mathrm{~km} \mathrm{~s}^{-1}$ range is seen mostly east-south from the edge of the stellar disk; a similar feature is seen in maps of other species of moderate-to-high excitation, for instance, in the $\mathrm{H}_{2} \mathrm{O} 17_{4,13} \rightarrow 16_{7,10}$ line from $E_{\mathrm{u}}=5781 \mathrm{~K}$. The maps of the redshifted emission in $\mathrm{TiO}$ and $\mathrm{H}_{2} \mathrm{O}$ are shown in Fig. 7. Because the absorption component is much stronger in the highly excited line of $\mathrm{H}_{2} \mathrm{O}$, the net line intensity is negative close to the direction toward the star and pure emission is seen only east from the star, by as far as 0.2 in the beam-smeared maps. The TiO emission is much less affected by the redshifted absorption and the full extent of the emission component can be seen directly in Fig. 7. The emission can be traced up to $0^{\prime \prime} 2$ from the star toward east and west. The emission is a signature of warm gas falling on the star. Because we measure only the tangential motion and away from the direction of the kinematical center, the material seen in emission must be falling on the star with velocities in excess of the maximal redshift $17 \mathrm{~km} \mathrm{~s}^{-1}$. Being faint, the TiO emission must arise from a portion of gas that is only a small fraction of the bulk gas seen in the main emission component. If the infall was spherically symmetric around the star, the redshifted emission and absorption components seen above $56 \mathrm{~km} \mathrm{~s}^{-1}$ should have a corresponding blueshifted component at velocities below about $36 \mathrm{~km} \mathrm{~s}^{-1}$. However, it is not observed, most likely because that material is obscured by the radio photosphere.

We conclude that the submm features originate from gas located mainly in the extended atmosphere of Mira, at radii $\lesssim 4 R_{\star}$. The distribution of the bulk of TiO-bearing gas is more isotropic than that of $\mathrm{AlO}$ and is not directly affected by the pulsation shock that was active at the moment of the ALMA observations (Sect.4). A small fraction of TiO-bearing gas is also present 
in the infall. This infalling material may be warmer and more anisotropically distributed than the main mass of TiO.

\subsection{Spatio-kinematical characteristics $\mathrm{TiO}_{2}$-bearing gas}

The $\mathrm{TiO}_{2}$ lines appear as pure emission features (Fig. 3). However, because they are relatively weak, line blending is a problem in characterizing their profiles. To reduce the effect of line blending, we combined the six cleanest normalized lines into a median profile shown in Fig. 8. Strikingly, it displays two extended wings. In the $9_{5,5}-8_{4,4}$ transition alone, compared to the median profile in Fig. 8, the red wing is completely missing and weak absorption or zero level is seen instead. It is possible that part of the $\mathrm{TiO}_{2}$ gas is present in the infall but the corresponding absorption component is too weak to produce an unambiguous absorption feature. The $\mathrm{TiO}_{2}$ profiles appear to be slightly broader for transitions from higher lying energy levels, but this trend may be mimicked by the heavy line crowding.

The broad non-Gaussian wings make $\mathrm{TiO}_{2}$ profiles distinctive from any profiles of other species observed. This is particularly apparent when the $\mathrm{TiO}_{2}$ profile is compared to that of TiO, as in Fig. 8. The typical FWHM of $8.5 \mathrm{~km} \mathrm{~s}^{-1}$ of the emission profile is however only slightly larger than that of $\mathrm{TiO}$ $\left(F W H M=7.5 \mathrm{~km} \mathrm{~s}^{-1}\right)$. While the cores of the $\mathrm{TiO}_{2}$ emission lines may originate from the same gas in the extended atmosphere, the material apparent in the wings should be characterized by an increased abundance of $\mathrm{TiO}_{2}$ relative to $\mathrm{TiO}$.

The emission region of $\mathrm{TiO}_{2}$ is larger than the ALMA beam in our Band 7 observations and has a beam-deconvolved full size of about $(159 \pm 10) \times(78 \pm 11)$ mas at $\mathrm{PA}=107^{\circ}$. These values were measured as a Gaussian fit to an image of seven combined $\mathrm{TiO}_{2}$ transitions to obtain a better $\mathrm{S} / \mathrm{N}$. In case the emission is actually patchy or multiple components are present, our Gaussian fit would yield an inadequate measure of the size. Nevertheless, the Gaussian area of the emission region of $\mathrm{TiO}_{2}$ is only $10 \%$ larger than that of $\mathrm{TiO}$ and it appears more asymmetric, i.e., has a higher eccentricity. The orientation of the long axis of the Gaussian fits is the same for both oxides. The comparison of the maps of $\mathrm{TiO}$ and $\mathrm{TiO}_{2}$ emission in Fig. 6 suggests that $\mathrm{TiO}_{2}$ emission is indeed more extended even if the difference in excitation and $\mathrm{S} / \mathrm{N}$ is taken into account. The centroid position of $\mathrm{TiO}_{2}$ emission is $13( \pm 2)$ mas north and $30( \pm 2)$ mas east from the continuum center, which is 1.6 times farther away from the star than what we measured for TiO. This displacement is apparent in Fig. 6. The location and distribution of the $\mathrm{TiO}_{2}$ resembles more that of warm $\mathrm{SO}_{2}$, which is representative of species present in the silicate formation zone and the wind.

The $\mathrm{TiO}_{2}$ traced in submm lines is located mainly in the extended atmosphere of Mira, in layers where we observe TiO, but its spatial distribution is more anisotropic than that of $\mathrm{TiO}$. With the radii $(F W H M / 2)$ of $2.7-5.5 R_{\star}$ of the emission region, a smaller amount of $\mathrm{TiO}_{2}$-bearing gas extends to farther distances from the star than the monoxide emission $\left(<4 R_{\star}\right)$. Overall, $\mathrm{TiO}_{2}$ resides in the quiescent part of the envelope that is not directly affected by the supersonic shock.

\subsection{Excitation and column densities of $\mathrm{TiO}$ and $\mathrm{TiO}_{2}$}

We used the rotational-diagram method (Goldsmith \& Langer 1999) to derive the temperature and column density of TiO that is traced in the main emission component. The diagram is shown in Fig. 9. A fit to all lines, including the line tentatively detected with Herschel, yielded an excitation temperature

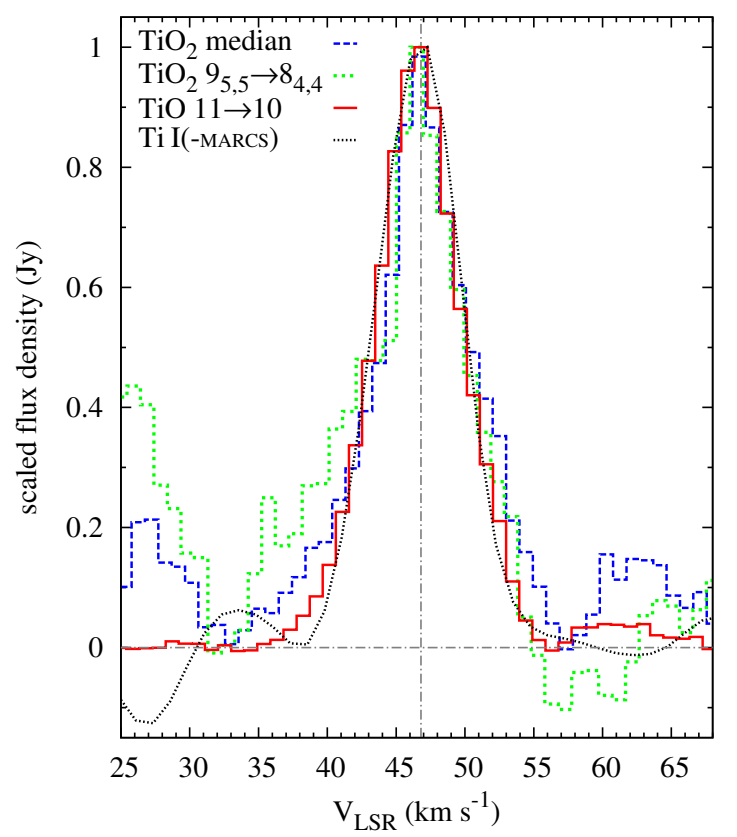

Fig. 8. Representative line profiles of Ti-bearing species in Mira. Ti I: an optical spectrum of Ti I $\lambda 5173$ is shown with dotted black line. It is an average of two spectra acquired for phases 0.17 and 0.24 and represents the phase of ALMA observations $(\varphi=0.21)$. To correct the average profile for the photospheric line of Ti I, a synthetic spectrum based on MARCS model atmospheres was subtracted. The subtraction is not perfect and the profile of circumstellar Ti I is distorted by photospheric features of TiO. TiO: our best ALMA observations of the pure rotational line of $\mathrm{TiO}$ is shown as a red histogram. $\mathrm{TiO}_{2}$ : two profiles are shown for $\mathrm{TiO}_{2}$; one is a median of six normalized transitions and represents lines of different excitation potentials (blue dashed histogram); the other shows a profile of the $9_{5,5}-8_{4,4}$ transition (gray dotted histogram).

$T_{\text {ex }}=491 \pm 96 \mathrm{~K}$ and a column density $N\left({ }^{48} \mathrm{TiO}\right)=(4.0 \pm 1.0) \times$ $10^{16} \mathrm{~cm}^{-2}$ for a source size of $116 \times 93$ mas. The errors quoted here represent only the quality of the fit. The values of $T_{\mathrm{ex}}$ and $N$ are derived under the assumption that all lines are optically thin, the level populations follow the Boltzmann distribution, the rotational temperatures in the three fine-structure components are equal, and the line fluxes do not change with time or phase so that data from different epochs and different spin components can be combined. The rotational lines of $\mathrm{TiO}$ are not formed in the immediate proximity of shocked regions (Sect. 5.3) so LTE conditions are likely to apply. Some other assumptions might be violated in Mira, but our estimates here are the best constraints on $T_{\text {ex }}$ and $N$ that are possible with the current data.

After we constrained the excitation temperature of the main $\mathrm{TiO}$ isotopologue, we used the measured fluxes of the rare $\mathrm{TiO}$ species to calculate their relative abundances. This is carried out under the assumptions that the different species are characterized by the same temperature and there is no isotopic fractionation. Both conditions are very likely fulfilled in the atmosphere of Mira. The ratios with $1 \sigma$ uncertainties are given in the first row of Table 3 . In addition, we obtain ${ }^{47} \mathrm{Ti} /{ }^{48} \mathrm{Ti}<0.08$ from the flux ratio of the $\Delta_{3} J=11 \rightarrow 10$ transition of ${ }^{48} \mathrm{TiO}$ and the upper limit of ${ }^{47} \mathrm{TiO}$, corrected for the intrinsic lines strengths.

The excitation analysis of $\mathrm{TiO}_{2}$ is more difficult. Although many transitions in a broad range of $E_{\mathrm{u}}$ were covered, the severe blending of lines leaves only four relatively clean lines whose fluxes could be measured over the entire profile. The lines tentatively detected with APEX were not taken into account in our analysis here. A simple rotational-diagram fit to those four 


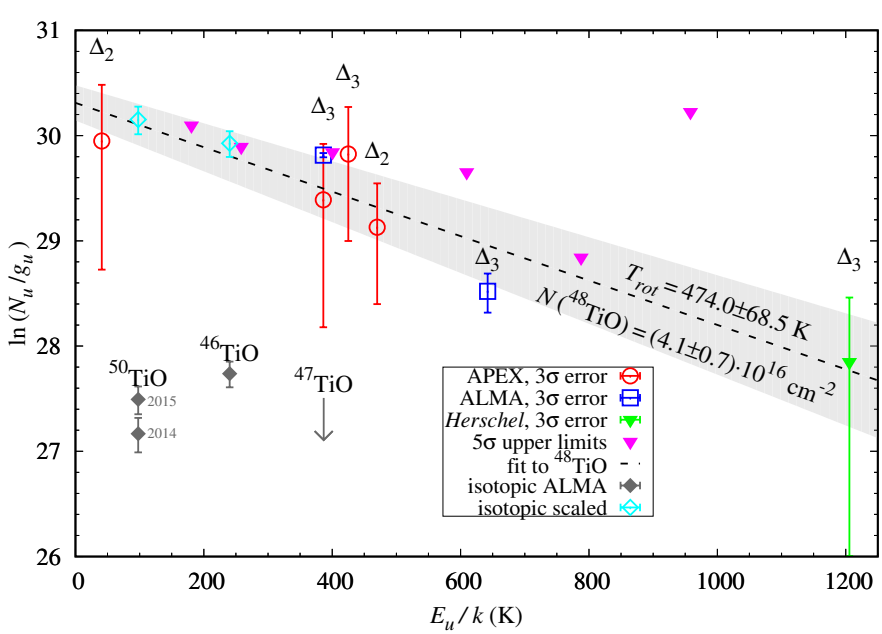

Fig. 9. Rotational diagram for TiO. Red, blue, and green points represent detected lines and the magenta triangles indicate $5 \sigma$ upper limits. The linear fit that constrains the excitation temperature and column density of the main isotopologue, ${ }^{48} \mathrm{TiO}$ is shown with the dashed line and the shaded area corresponds to $1 \sigma$ uncertainty of the fit. Gray points and an arrow (lower left) correspond to measurements of ${ }^{46} \mathrm{TiO}$ and ${ }^{50} \mathrm{TiO}$ and to an upper limit for ${ }^{47} \mathrm{TiO}$. The two 2015 measurements for ${ }^{46} \mathrm{TiO}$ and ${ }^{50} \mathrm{TiO}$, scaled by reciprocal of their solar abundances, are shown with cyan diamonds. They were included in the final fit.

measurements yielded $T_{\text {rot }}=180 \pm 67 \mathrm{~K}$ and $N\left(\mathrm{TiO}_{2}\right)=(9.5 \pm$ $1.8) \times 10^{15} \mathrm{~cm}^{-2}$ (for a fixed source solid angle of $0.0138 \mathrm{mas}^{2}$ ). However, these results are not very satisfactory owing to the insufficient coverage of $E_{\mathrm{u}}$, i.e., only $60-137 \mathrm{~K}$, and the modest $\mathrm{S} / \mathrm{N}$ of the measurements. To make a better use of the data, we employed the LTE package of CASSIS ${ }^{3}$ and performed a $\chi^{2}$ minimization in which a grid of models was compared to ten observed line profiles. In the models, we approximated the profiles by a Gaussian with a FWHM of $8.5 \mathrm{~km} \mathrm{~s}^{-1}$ and a central velocity of $46.8 \mathrm{~km} \mathrm{~s}^{-1}$. Only the uncontaminated parts of the profiles were included in the minimization procedure (Fig. 3). In the CASSIS approach, we also used the information on lines that were not detected extending the analysis to transitions from energy levels as high as $E_{\mathrm{u}}=904 \mathrm{~K}$. Optical-depth effects were taken into account in the routine, but the lines turned out to be optically thin (with $\tau_{0} \leq 1.0$ for line centers). The parameters best characterizing the $\mathrm{TiO}_{2}$ gas, $T_{\text {rot }}=174 \pm 7 \mathrm{~K}$ and $N\left(\mathrm{TiO}_{2}\right)=(1.15 \pm 0.12) \times 10^{16} \mathrm{~cm}^{-2}(1 \sigma$ errors $)$, were used to simulate the spectrum shown in Fig. 3. The single-component model reproduces the data relatively well but the observed line profiles are not exactly Gaussian. It is possible that our isothermic model is not adequate for the $\mathrm{TiO}_{2}$ gas, but the quality of data and their spread in parameters space do not warrant multicomponent models.

With the temperature constrained for $\mathrm{TiO}_{2}$, we next used CASSIS to derive the column densities for the rare isotopologues. By analyzing the two lines of ${ }^{46} \mathrm{TiO}_{2}$, we derived $N\left({ }^{46} \mathrm{TiO}_{2}\right)=(3.7 \pm 0.3) \times 10^{15} \mathrm{~cm}^{-2}$ or the abundance ratio of ${ }^{46} \mathrm{TiO}_{2} /{ }^{48} \mathrm{TiO}_{2}=0.32 \pm 0.04$. This result is not fully satisfactory because our fit to ${ }^{48} \mathrm{TiO}_{2}$ profiles is particularly imperfect in the line center, which dominates the profile of the low $\mathrm{S} / \mathrm{N}$ features of ${ }^{46} \mathrm{TiO}_{2}$ (cf. Figs. 3 and 4). From half a profile of the only feature of ${ }^{50} \mathrm{TiO}_{2}$, we obtain an upper limit $N\left({ }^{50} \mathrm{TiO}_{2}\right) \leq$ $2.9 \times 10^{15} \mathrm{~cm}^{-2}$ or ${ }^{50} \mathrm{TiO}_{2} /{ }^{48} \mathrm{TiO}_{2} \leq 0.25$. Upper limits on several transitions of ${ }^{47} \mathrm{TiO}_{2}$ imply $N\left({ }^{47} \mathrm{TiO}_{2}\right)<6 \times 10^{14} \mathrm{~cm}^{-2}$ or ${ }^{47} \mathrm{TiO}_{2} /{ }^{48} \mathrm{TiO}_{2}<0.05$. Similarly, for ${ }^{49} \mathrm{TiO}_{2}$ we were only

3 http://cassis.irap.omp.eu
Table 3. Isotopic ratios.

\begin{tabular}{ccccc}
\hline \hline Method & ${ }^{46} \mathrm{Ti} /{ }^{48} \mathrm{Ti}$ & ${ }^{47} \mathrm{Ti} /{ }^{48} \mathrm{Ti}$ & ${ }^{49} \mathrm{Ti} /{ }^{48} \mathrm{Ti}$ & ${ }^{50} \mathrm{Ti} /{ }^{48} \mathrm{Ti}$ \\
\hline TiO rot.diagr. & $0.12 \pm 0.03$ & $<0.12$ & & $0.08 \pm 0.02$ \\
TiO line ratio & & $<0.08$ & & \\
$\mathrm{TiO}_{2}$ CASSIS & $0.32 \pm 0.04^{a}$ & $<0.05$ & $<0.04$ & $\leq 0.25$ \\
$\mathrm{TiO}_{2}$ line ratio & & $<0.06$ & $<0.08$ & $<0.22$ \\
Solar & 0.11 & 0.10 & 0.07 & 0.07 \\
\hline
\end{tabular}

Notes. The uncertainties are $1 \sigma$ random errors. ${ }^{(a)}$ An uncertain result with high systematic errors.

able to derive an upper limit, $N\left({ }^{49} \mathrm{TiO}_{2}\right)<5 \times 10^{14} \mathrm{~cm}^{-2}$ or ${ }^{49} \mathrm{TiO}_{2} /{ }^{48} \mathrm{TiO}_{2}<0.04$. These CASSIS results, summarized in the third row of Table 3 , take $1 \sigma$ noise levels into account and do not suffer from the low $\mathrm{S} / \mathrm{N}$ problem or from saturation effects as in the rotation-diagram method.

The isotopic ratios can also be derived by comparing the same transition of the rare isotopologue to that of ${ }^{48} \mathrm{TiO}_{2}$. Such a direct comparison is possible for $12_{4,8} \rightarrow 11_{3,9}$ of ${ }^{50} \mathrm{TiO}_{2}$, $10_{5,5} \rightarrow 9_{4,6}$ of ${ }^{49} \mathrm{TiO}_{2}, 9_{5,5} \rightarrow 8_{4,4}$ of ${ }^{48} \mathrm{TiO}_{2}$. Although the transition $23_{3,21} \rightarrow 22_{2,20}$ of both ${ }^{46} \mathrm{TiO}_{2}$ and ${ }^{48} \mathrm{TiO}_{2}$ was observed, the direct flux ratio does not give a good measure of their relative abundances because the ${ }^{48} \mathrm{TiO}_{2}$ line is contaminated by emission of an unknown origin. The results are given in the fourth row of Table 3. The isotopic ratios derived this way are independent of the actual temperature but may in principle be underestimated owing to opacity effects.

All the constraints on the isotopic ratios are summarized in Table 3. Within errors, the combined results are indicative of relative abundances that are not drastically different from solar values except for ${ }^{47} \mathrm{Ti}$, which may be underabundant by a few percent. We took advantage of this result to improve our temperature and column density determination for TiO. We scaled the 2015 ALMA flux measurements for ${ }^{46} \mathrm{TiO}$ and ${ }^{50} \mathrm{TiO}$ by their abundances relative to ${ }^{48} \mathrm{TiO}$ and included them in the rotational diagram fit of $\mathrm{TiO}$. This improved the quality of the fit and yielded $T_{\mathrm{ex}}=474 \pm 69 \mathrm{~K}$ and $N\left({ }^{48} \mathrm{TiO}\right)=(4.1 \pm 0.7) \times$ $10^{16} \mathrm{~cm}^{-2}$, which we treat as our best estimates for TiO.

The abundances of $\mathrm{TiO}$ and $\mathrm{TiO}_{2}$ with respect to hydrogen are useful for further discussion. As discussed in Paper I, there is currently no reliable way to estimate the content of hydrogen in the probed regions. Nevertheless, hydrodynamical models of Mira of the CODEX grid (Ireland et al. 2011) give the hydrogen column density in the range $10^{22}-10^{24} \mathrm{~cm}^{-2}$, which indicates $\mathrm{TiO}$ abundance of $10^{-8}-10^{-6}$. Values higher than $10^{-7}$ are unrealistic as such values would require the elemental abundance of titanium to be higher than the cosmic value $\left(1.0 \times 10^{-7}\right)$. The source-averaged abundances of $\mathrm{TiO}$ and $\mathrm{TiO}_{2}$ must be therefore on the order of $10^{-8}-10^{-7}$, but their uncertainties are uncomfortably large. No reliable excitation analysis was possible for the warm TiO component seen in the infall and the - presumably cool - gas manifested in the broad wings of $\mathrm{TiO}_{2}$.

\section{Optical spectroscopy of Ti-bearing species}

\subsection{Neutral atomic titanium}

Several electronic multiplets of Ti I are observed in absorption in the optical spectra of Mira, including some resonance transitions. Examples are presented in Figs. C.1-C.5 of Paper I; all these examples are photospheric lines. One striking exception of a line that does not appear as a pure absorption feature 
is the resonance line $a^{3} F_{2}-z^{3} F_{3}$. Its upper energy level at $E_{\mathrm{u}}=27800 \mathrm{~K}$ (Forsberg 1991), is the lowest among all covered transitions of Ti I. The line is dominated by emission in a great majority of our multi-epoch spectra. With a laboratory wavelength of $5173.74 \AA$, the line is located within the deep absorption band $\alpha(0,0)$ of TiO. The molecular lines are saturated and tightly overlap with each other making the pseudo-continuum relatively flat in this spectral range. Owing to this location, Ti I $\lambda 5173$ is the most conspicuous line in the $a^{3} F-z^{3} F$ multiplet.

At close inspection, the feature has an inverse P-Cyg type profile. Although such a profile usually indicates infall, in this case the feature is formed mainly by an emission profile overlapping with a photospheric absorption line of Ti I. The photospheric origin of the absorption is supported by (1) the presence of pure absorption profiles in the majority of Ti I multiplets observed; (2) the presence of an absorption line of nearly the same intensity and width in stationary model atmospheres of MARCS (Gustafsson et al. 2008) and for stellar parameters adequate for Mira; and (3) the central velocity of the absorption feature, corrected for the overlapping emission, corresponds well to the velocity of the optical photosphere. Inverse P-Cyg profiles of nonresonant $\left(E_{\mathrm{u}}=21760\right.$ and $\left.25100 \mathrm{~K}\right)$ lines of $\mathrm{Ti}$ I were also observed in the near-infrared (Ferlet \& Gillet 1984) but were incorrectly interpreted as direct signatures of infall. A profile of the $\mathrm{Ti}$ I emission line is compared to pure rotational features of $\mathrm{TiO}$ and $\mathrm{TiO}_{2}$ in Fig. 8. This profile is an average of two Ti I spectra taken at phases 0.17 and 0.24 in 2010 from which we subtracted a photospheric spectrum. The latter was a MARCS synthetic spectrum shifted to a velocity of $V_{\mathrm{LSR}}=47.6 \mathrm{~km} \mathrm{~s}^{-1}$. At this velocity the simulated electronic structure of the $\mathrm{TiO} \alpha(0,0)$ band matches best the observations ${ }^{4}$. The center and width of the Ti I emission at phase $\sim 0.2$ is the same as for the majority of molecular emission features arising in the extended atmosphere and traced with ALMA. In particular, the profile is almost the same as that of the pure rotational line of TiO.

The Ti I $\lambda 5173$ emission is seen in every pulsation cycle and therefore is not an episodic phenomenon as some Al-bearing emission features reported in Paper I. When analyzed in the multi-epoch spectra, the emission intensity relative to that of continuum shows considerable variations. The relative intensity is lowest at visual maxima and systematically increases with phase reaching a maximum of $130 \%$ near visual minima. Those changes are modulated by the continuum flux variations by huge factors of 158-1580 (5.5-8.0 mag), depending on the cycle (Paper I). The Ti I emission follows these dramatic changes also displaying intrinsic line variations at a level of $30 \%$. Absolute intensities of the emission component cannot be measured directly in our spectra owing to unreliable flux calibration and an uncertain contribution of the photospheric component in each phase.

The apparent peak of the Ti I emission profile changes its position over time between $V_{\mathrm{LSR}}=43.8$ and $47.3 \mathrm{~km} \mathrm{~s}^{-1}$ with no obvious correlation with phase. These variations are most likely caused by the overlap of the absorption and emission components, which both vary in time. Near maximum visual light, when the emission component is weak with respect to the continuum and with respect to the photospheric line, the absorption component dominates the entire profile shifting the net emission peak toward shorter wavelengths. The intrinsic changes in the velocity of the pure emission component must thus be considerably smaller than $3.5 \mathrm{~km} \mathrm{~s}^{-1}$ and, in fact, we believe that the line

\footnotetext{
4 Note that optical photosphere is not expected to be stationary and therefore its velocity is not necessarily the same as that of the center of mass.
}

is stationary. It is most apparent in the photosphere-corrected profile shown in Fig. 8, which is centered at the stellar centerof-mass velocity. In profiles dominated by the emission component, the FWHM is of $5.5 \pm 0.5 \mathrm{~km} \mathrm{~s}^{-1}$ and the full base width is of about $11 \mathrm{~km} \mathrm{~s}^{-1}$, which are characteristic for the material in the extended atmosphere seen with ALMA data at radii $\lesssim 4 R_{\star}$.

To produce the Ti I $\lambda 5173$ transition in emission, it may seem necessary to populate its upper energy level, which is $27800 \mathrm{~K}$ above the ground. Direct collisional excitation of this state can be entirely excluded. Although the pulsation shock is capable of producing such high-excitation conditions, any shock-triggered emission would show dramatic changes in intensity and velocity that are not observed in Ti I. For the same reasons we can exclude the influence of the radiation of the shock, whether it is a direct excitation or fluorescence (cf. Willson 1976).

An alternative mechanism that can explain the Ti I emission is resonant scattering. The random shifts in the centroid position of Ti I with a low amplitude of $<3.5 \mathrm{~km} \mathrm{~s}^{-1}$, if present, could be partially explained by an inhomogeneous and variable distribution of the scattering medium. Such asymmetries have been observed close to the photosphere of Mira in the distribution of gas species (Paper I) and hot dust (Lopez et al. 1997). Additionally, for resonant scattering one would expect the scattered flux to be tightly correlated with the intensity of the continuum and, as a consequence, the continuum-normalized line intensity should be roughly constant with phase. Such a correlation is observed in $\lambda 5173$ but is not strict. Considering the enormous visual light variations of Mira with flux changing by a factor of 250 for the epochs covered by our optical spectra, the reported variability of $30 \%$ in the continuum-normalized line flux can be considered insignificant and may be partially explained by line-saturation effects. Resonant scattering is therefore a reasonable explanation of the Ti I emission.

The resonantly scattered emission of Ti I does not require high electron densities and temperatures but requires sufficient population of the ground level. This requirement suggests lowexcitation conditions for the Ti I-bearing gas. The two strongest lines of the multiplet to which $\lambda 5173$ belongs have their lower levels at $245 \mathrm{~K}$ and $557 \mathrm{~K}$. We did not analyze these lines here because they are severely distorted by overlapping $\mathrm{TiO}$ features. It is evident, however, that they produce strong emission features similar to that of 25173 . At the same time, Ti I multiplets with higher lying levels are only seen in absorption. Although we are unable to calculate the excitation temperature because of the heavy line blending, we can conclude that the Ti I emission must arise from circumstellar material of a temperature of a few hundred $\mathrm{K}$. The sole fact that we see the TiI emission within the saturated $\alpha$ band of TiO implies that the Ti-bearing gas must be located above the absorbing layers of TiO. This electronic molecular band is opaque enough to absorb any radiation from layers lying beneath its carrier. The most likely location of Ti I is the extended atmosphere of Mira.

\subsection{Titanium ion, Till}

We were unable to identify any spectral features indicative of significant amounts of Ti II in the envelope of Mira. Our optical spectra cover only one ground-state transition of Ti II, which is a forbidden transition, however, and is not useful for probing the dense shock-excited photospheric gas in which this ion is expected to reside (Willson 1976). We found a weak emission feature at the position of the [Ti II] line (7916.2 $\AA$ corrected to the center-of-mass velocity), but we were unable to establish 


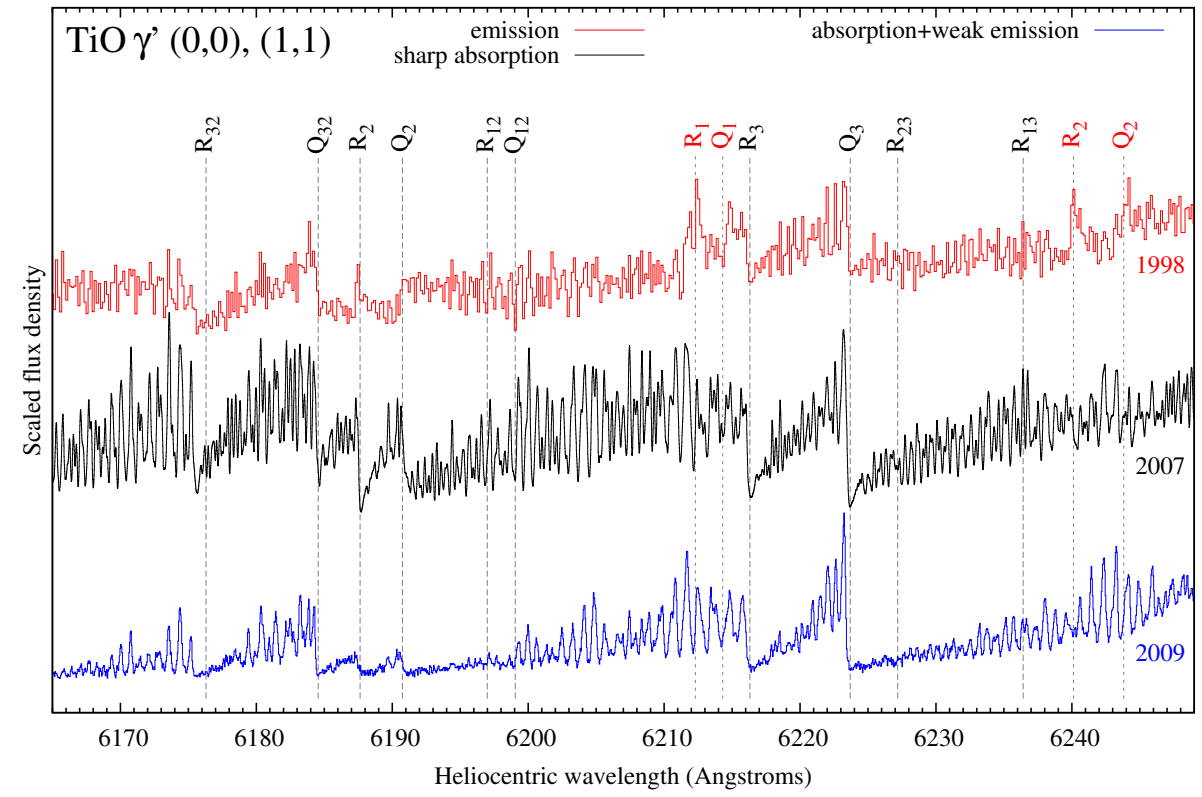

Fig. 10. Sample spectra of the $\mathrm{TiO} \gamma^{\prime}(0,0)$ and $(1,1)$ bands illustrating the emission-dominated bands (top, red; 7 Nov. 1998), sharp absorption band-heads (middle, black; 20 Jan. 2007), and absorption bands partially filled with emission (bottom, blue; 8 Aug. 2009). The full collection of spectra is shown in Fig. C.1. Main TiO features are identified with vertical dashed lines, with black and red labels corresponding to the $(0,0)$ and $(1,1)$ bands, respectively. whether it is the atomic line or a rotational line of overlapping molecular bands. Fox et al. (1984) postulated the presence of the Ti II line at $4307.9 \AA$, which should be blended with a line of Fe I. We found a strong emission feature in some of our spectra at this position, but other lines of the same Ti II multiplet are missing. This raises questions about this identification. Archival ultraviolet spectra of Mira, for example, those of Karovska et al. (1997), cover a strong resonance line of Ti II at $3383.8 \AA$, but do not show anything conspicuous at this position, possibly owing to the lack of spectral resolution and sensitivity.

We conclude that the observations in hand are not well suited to conclusively investigate the presence of titanium ions in Mira, but it is very likely that their abundances are negligible.

\subsection{TiO emission}

The absorption spectrum of $\mathrm{TiO}$ was recognized in $o$ Ceti a century ago (Fowler 1909). These variable bands are formed in the photosphere and are responsible for the dramatic changes in the visual light of Mira over its 330-day period (Reid \& Goldston 2002). It is difficult to identify any circumstellar features in the absorption spectra because of a very complex structure of the numerous and overlapping photospheric bands and because of incomplete laboratory measurements of $\mathrm{TiO}$ line positions.

Circumstellar material is manifested in electronic bands when they are seen in emission. In Paper I, we reported emission in optical bands of $\mathrm{AlO}$, which is very episodic and may be related to shock phenomena. From the plethora of electronic bands of $\mathrm{TiO}$ covering the entire optical region, we noticed emission features only in the $\gamma^{\prime}(\equiv B-X)(0,0)$ and $(1,1)$ bands between 6170 and $6250 \AA$. All spectra covering this range are shown in Fig. C.1. Strong emission was only observed once in the 35 spectra. There are, however, hints that weak emission appears in the same bands much more often but is almost completely consumed by the overlapping photospheric absorption. Examples of $\mathrm{TiO}$ spectra affected by emission and free of it are shown in Fig. 10.

Strong and obvious emission of $\gamma^{\prime}$ was observed on 7 November 1998 near a visual phase of 0.9. Such TiO flaring must be extremely rare. On the same date, the AlO bands were also seen in emission (Paper I). The TiO emission is most apparent in the $(1,1)$ transitions, in particular involving the $R_{1}$ and $R_{2}$ branches (at $\sim 6212$ and $\sim 6240 \AA$ in Fig. 10). It is also evident near the $R_{2}$ bandhead of $(0,0)(\sim 6188 \AA)$. In addition, many of the $\gamma^{\prime}(0,0)$ absorption bandheads appear much shallower than in a typical spectrum of Mira at the same phase because they are filled with emission components, as illustrated in Fig. 10. The $\mathrm{S} / \mathrm{N}$ of the emission-dominated features is too low to constrain the temperature and kinematics of the emitting gas. However, from the appearance of the $(1,1)$ emission we can infer that the emitting gas must have a temperature lower than about $1000 \mathrm{~K}$ because otherwise emission would arise in a broader range of rotational transitions.

Other identified emission features are much less conspicuous. The TiO bands usually show sharp triangular absorption bandheads that develop toward longer wavelengths, but beginning 29 October 2008 the absorption heads became blunt and remained so until at least 2012. The last spectrum in our collection, from 2014, shows the sharp absorption heads back again. Two example spectra of sharp and blunt heads are compared in Fig. 10 (black and blue, respectively). The difference is most pronounced in $R_{2}(6188 \AA)$ and $Q_{2}(6224 \AA)$ and bandheads of $(0,0)$. When the spectra affected by weak emission are analyzed one by one in the chronological order (Fig. C.1), some variability in the emission component can be seen.

The upper electronic level of $\gamma^{\prime}$ at $E_{\mathrm{u}} \approx 22800 \mathrm{~K}$ is below that of the optical AlO band $\left(E_{\mathrm{u}} \approx 28800 \mathrm{~K}\right)$ which was identified in emission in Paper I. Our spectra cover electronic systems of $\mathrm{TiO}$ that have their upper levels lying above (e.g., $a-f$ and $\alpha$ ) and below (e.g., $\gamma$ and $\epsilon$ ) that of $\gamma^{\prime}$ but none shows signs of emission. It is unclear why the emission episode was limited to one electronic system of TiO. The emission cannot be associated with a regular pulsation shock because then it would appear in every cycle. On the other hand, there are no other potential sources that could produce emission from such high-lying energy levels by collisional excitation only. Taking the episodic nature of the strong emission event and its similarity to what was observed in electronic bands of $\mathrm{AlO}$, we propose that the emission is triggered by an unusually strong shock that excites material in the parts of the atmosphere that are quiescent in a regular cycle (cf. Paper I, Richter et al. 2003).

The origin of the weaker emission in the $\gamma^{\prime}$ band of TiO, which appears in the recorded spectra much more often, may 
be different. Resonantly scattered emission seems a reasonable mechanism to explain it. The inhomogenities present in the closest vicinity of the star and the ever-changing distribution of gas around the stellar photosphere can be responsible for the variable nature of this weak emission component. One can further speculate that the emission episode from 6 November 1998 was an extreme case of this behavior and geometrical asymmetries produced a large volume of TiO-bearing gas surrounding the stellar disk that by far suppressed the absorption. However, this scenario does not explain why the emission appeared in only one electronic system.

Mira was observed close to its 1990 maximum using techniques of aperture-masking interferometry by Haniff et al. (1992). The object was imaged at a resolution of $\sim 30$ mas in the pseudocontinuum at $7007 \pm 4 \AA$ and within a spectral region $7099 \pm 4 \AA$, which is dominated by the TiO $\gamma(0,0)$ band. The source imaged within the $\mathrm{TiO}$ band appeared $\sim 1.5$ times larger than in pseudocontinuum. That led Haniff et al. to a conclusion that there is a halo of resonantly scattered emission of $\mathrm{TiO}$ around the stellar photosphere. The $\gamma$ system has the upper electronic level $20220 \mathrm{~K}$ above the ground, only slightly below that of $\gamma^{\prime}(0,0)$ discussed above. It is unlikely that the imaging observation caught the star during an episode of strong $\mathrm{TiO}$ emission, like the one seen in 1998 in $\gamma^{\prime}$. Rather, it was emission analogous to the $\gamma^{\prime}$ features that we observed in 2008-2012. These interferometric observations support our interpretation of the weak TiO emission as resonantly scattered continuum. We do not see the emission component of $\gamma$ bands in our spectra owing to insufficient angular resolution. The interferometric data also locate the scattering medium at a radius of $\sim 3 R_{\star}$ and demonstrate that the distribution of TiO has some degree of anisotropy (their Fig. 3d). The circumstellar emission of TiO seen at optical wavelengths is therefore at least partially collocated with that traced in the pure rotational lines at submm wavelengths in Mira's extended atmosphere (cf. Sect. 5.3).

TiO isotopologues. Using our optical spectra, we attempted to constrain the isotopic ratios of the different TiO isotopologues in a similar fashion as in Wyckoff \& Wehinger (1972) and Chavez \& Lambert (2009). Even in the spectra of highest dispersion, however, the isotopic effects are too subtle to reliably measure contributions of the different isotopic species. In a procedure described in Appendix B, we found that the overall enhancement of the four rare isotopologues must be less than about $25 \%$.

\section{4. $\mathrm{TiO}_{2}$ absorption}

One known electronic system of $\mathrm{TiO}_{2}, \tilde{A}^{1} B_{2}-\tilde{X}^{1} A_{1}$, is located between about 5360 and $5450 \AA$ (Zhuang et al. 2010; Wang et al. 2009). This system overlaps with part of the $\Delta v=-2$ progression of $\mathrm{AlO} B-X$ (very weak in Mira) and is located within the $\gamma^{\prime}(3,0)$ band of TiO. Presumably, the dominant source of opacity at these wavelengths is $\mathrm{TiO}$, making the identification of any $\mathrm{TiO}_{2}$ features very challenging. We performed a simulation of the $\mathrm{TiO}_{2}$ bands in pgopher (Western 2014) using spectroscopic constants from the literature. Although the spectroscopic parameters presented in Wang et al. (2009) do not reproduce the full spectrum of $\mathrm{TiO}_{2}$ generated in laboratory conditions. The simulation shows a profile whose general shape corresponds well to what is seen in the spectra of Mira at different phases of its cycle. However, discrete features of $\mathrm{TiO}_{2}$ are not conspicuous in the observed spectrum, suggesting that $\mathrm{TiO}_{2}$ bands are not present. A full model-atmosphere spectrum with a complete line list of $\mathrm{TiO}$ is necessary to verify the presence of $\mathrm{TiO}_{2}$ in this spectral range. The published spectroscopic parameters we used represent only a small part of the electronic spectrum of $\mathrm{TiO}_{2}$ and more spectroscopic work is necessary to verify whether bands of $\mathrm{TiO}_{2}$ are present in optical spectra of stars.

\subsection{TiH absorption}

To investigate the presence of $\mathrm{TiH}$ in the spectra of Mira we simulated the optical bands in pgopher. We used the line list $^{5}$ compiled by $\mathrm{Z}$. Yueqi $\mathrm{Na}$ for the ExoMol project (Tennyson \& Yurchenko 2012) and based on the spectroscopic data of Burrows et al. (2005). In a broad range of temperatures characteristic of the circumstellar gas and the photosphere, the strongest optical bands of TiH are the $B^{4} \Gamma-X^{4} \Phi$ and $A^{4} \Phi-X^{4} \Phi$ systems at about 5100-5385 $\AA$ and 9380-9700 $\AA$. A direct comparison between the simulations and observed spectra at different phases did not reveal any conspicuous features of $\mathrm{TiH}$. These spectral ranges are dominated by the photospheric bands of $\mathrm{TiO} \gamma^{\prime}, \mathrm{AlO} B-X$, and $\mathrm{H}_{2} \mathrm{O}$ (including the telluric bands). A comprehensive test for the presence of $\mathrm{TiH}$ would require removing the contribution of the other species to the observed spectra, but this is currently not feasible because of the same reasons as mentioned for $\mathrm{TiO}_{2}$. Our analysis shows, however, that TiH cannot be a major Ti carrier in Mira and its envelope.

For the other potential molecular carriers of Ti mentioned in Sect. 2, we could not find appropriate spectroscopic data (TiN, $\mathrm{TiF}, \mathrm{TiF}_{2}$ ) or the spectral signatures are located in ranges that were not covered by our spectra (TiS).

\section{Discussion}

\subsection{Tracing titanium chemistry in O-type AGB stars}

Our multiwavelength observations of Mira allow us to trace the Ti-bearing species in the different phases of the photospheric and circumstellar gas. We compare the gas characteristics deduced from the observations with chemical models to better constrain the titanium chemistry in an O-rich star like Mira.

Chemical abundances in the photosphere are thought to be reproduced well by predictions of equilibrium chemistry (Tsuji 1973; Cherchneff 2006). At the photospheric temperature of Mira of $\sim 3000 \mathrm{~K}$, titanium should be locked in TiO and in nearly equally abundant atomic Ti (e.g., Tsuji 1973; Sharp \& Huebner 1990) with a negligible contribution of $\mathrm{TiO}_{2}$. For solar composition, the abundance of $\mathrm{TiO}$ is expected to be $10^{-7}$ with respect to $\mathrm{H}_{2}$. The atomic $\mathrm{Ti}$ and $\mathrm{TiO}$ in the photosphere produce very conspicuous and well-known spectral features. Although we did not attempt to derive the photospheric abundances, all our observations are consistent with the dominant presence of $\mathrm{TiO}$ and Ti I in the photosphere.

The circumstellar chemistry of titanium is more complex. In first attempts to understand circumstellar chemistry, it has often been assumed that there is a smooth transition in abundances from the photosphere to the extended atmosphere and to the wind, and that all the abundances are given by the TE conditions (e.g., Tsuji 1973; Sharp \& Huebner 1990; Gail \& Sedlmayr 1998). However, this approach was refuted on observational and theoretical grounds in pulsating Mira stars, whose atmospheres are strongly affected by the periodic passage of shocks and which

\footnotetext{
5 http://www. exomol.com/data/molecules/TiH/48Ti-1H/ Yueqi/
} 
effectively produce molecules that are predicted to be absent in TE (Cherchneff 2006, 2012, and references therein). Several models based on chemical kinetics aimed to describe and reproduce the molecular abundances of the shocked regions in O-rich AGB stars, including Duari et al. (1999), Cherchneff (2006) and Gobrecht et al. (2016). The latter study presents a model of the Mira star, IK Tau, with an effective temperature of $2200 \mathrm{~K}$ which is slightly lower than that of Mira, 2200-3200 K (cf. Paper I). We assume here that this model is representative of the chemistry of the Mira's envelope and compare the model predictions with our observational constraints.

Photospheric $\mathrm{TiO}$ is expected to be destroyed by the supersonic shock and reformed in the cooling wake of the shock. We do not observe any signatures of shock-excited $\mathrm{TiO}$ molecules. Although our submm observations reveal the presence of hot molecular gas from the post-shock region through transitions of $\mathrm{HCN}$ and $\mathrm{H}_{2} \mathrm{O}$ (Sect. 4), we do not observe TiO from there. However, our observations are essentially limited to a single phase and the strongest $\mathrm{TiO}$ emission may occur at earlier phases when the shock is penetrating deeper layers of the star that are obscured by the radio photosphere. In Sect. 6.3 we reported one instance of optical observation of TiO bands suggesting strong influence of shocks, but this must have been a manifestation of a phenomenon occurring only in some cycles, and possibly only those characterized by exceptionally strong shocks. Also, these emission episodes likely affect the gas of the extended atmosphere where shocks are too weak to directly destroy strongly bound molecules such as TiO.

If $\mathrm{TiO}$ is fully destroyed by the supersonic shock, it reforms in the post-shock material in the photosphere. The production of $\mathrm{TiO}$ from $\mathrm{Ti}$ I continues in the extended atmosphere whose lower temperature allows for oxidation of titanium. The formation of TiO decreases the content of Ti I. In general, the lower the temperature the higher the consumption of $\mathrm{Ti} I$ in the extended atmosphere. Because the temperature changes with phase, the abundance of atomic $\mathrm{Ti}$ is expected to undergo cyclical changes and its highest abundance is expected close to a visual maximum. Our optical observations reveal that TiI is present in the extended atmosphere (Sect. 6.1), but we could not quantify how much of Ti remains in the atomic gas. We can state with confidence, however, that the oxidation in the circumstellar medium is not $100 \%$ efficient. In addition, we observe cyclical variability in the emission features of TiI, but we are unable to ascribe them to the potential changes in the abundance. The model of Gobrecht et al. (2016) predicts variations of the Ti I abundance in the extended atmosphere of two orders of magnitude (Cherchneff, priv. comm.), which is comparable to the order of the optical light variations. The high-amplitude flux changes mask the intrinsic variability of the circumstellar features of Ti I observed by us. Overall, the model predictions are in good quantitative agreement with our observations of Ti I.

In cool circumstellar gas, the abundance of $\mathrm{TiO}$ should increase with radius at the expense of TiI. On the other hand, at low temperatures $\mathrm{TiO}$ is converted to $\mathrm{TiO}_{2}$. For reference, in TE the two oxides have comparable abundances at 1380 K (Gail \& Sedlmayr 1998). In the non-equilibrium model of Gobrecht et al. (2016), both conversion processes are insignificant for the overall content of $\mathrm{TiO}$ and its abundance remains nearly constant throughout the extended atmosphere and does not vary significantly with phase. For an abundance on the order of $10^{-7}$, i.e., close to the TE abundance, $\mathrm{TiO}$ is expected to be the dominant molecular carrier of $\mathrm{Ti}$ and we observe considerable amounts of $\mathrm{TiO}$ in the atmosphere of Mira at submm and optical wavelengths. Our abundance estimate, although highly uncertain, is of the same order of magnitude as predicted in the model. The bulk of TiO-bearing gas has an excitation temperature of $475 \mathrm{~K}$, which is indicative of a location within the extended atmosphere. The ALMA observations allow us to trace the beam-smeared emission out to a radius of 240 mas or $17 R_{\star}$ (as measured for an isophote corresponding to the $3 \sigma$ noise level) so some amount of gaseous $\mathrm{TiO}$ may be present in the silicate-formation zone and in the wind. Beyond that region, the TiO-bearing gas is not excited sufficiently to produce conspicuous emission or it is depleted. Our observations do not indicate significant variability in the rotational lines of $\mathrm{TiO}$, which confirms the phase-invariant abundance predicted by Gobrecht et al. (2016).

The model of Gobrecht et al. (2016) does not predict significant production of $\mathrm{TiO}_{2}$ in the extended atmosphere. The small quantities that are produced are related to shock chemistry. At $6 R_{\star}$, Gobrecht et al. predict a $\mathrm{TiO}_{2}$ abundance that is two orders of magnitude lower than that of TiO. Although the relative contribution of $\mathrm{TiO}_{2}$ increases with distance from the star, it is a minor carrier of titanium in the model. Our observations indicate that although the $\mathrm{TiO}_{2}$ content is smaller than that of $\mathrm{TiO}$, the difference is not as extreme as in the simulations: the observed source-averaged column density ratio is $\mathrm{TiO} / \mathrm{TiO}_{2}=3.4 \pm 0.9$. The main emission components of both oxides have almost the same spatial extent and kinematics, indicating that they largely coexist in the same region of the extended atmosphere. However, the $\mathrm{TiO}_{2}$ emission region appears slightly more extended in some directions, its spectral features are slightly wider in velocity and its excitation temperature of $174 \mathrm{~K}$ is significantly lower than that of $\mathrm{TiO}$, indicating that there are parcels of gas where the $\mathrm{TiO}_{2}$-to- $\mathrm{TiO}$ ratio is increased. These $\mathrm{TiO}_{2}$-enhanced regions must be located farther from the star, in agreement with $\mathrm{TiO}_{2}$ forming directly from $\mathrm{TiO}$ and with the oxidation being more efficient farther from the star where the temperatures are lower (cf. Gail \& Sedlmayr 1998). The model is in general agreement with our observational constraints, except that $\mathrm{TiO}_{2}$ is produced more efficiently in Mira. An additional exploration of the model (Cherchneff, priv. comm.) shows that the $\mathrm{TiO}_{2}$ abundance is expected to change with phase by orders of magnitude; $\mathrm{TiO}_{2}$ is destroyed by the shocks and it takes about 0.2 of the period to reform the molecules in significant quantities. This behavior cannot be verified because our $\mathrm{TiO}_{2}$ data are limited to a single epoch.

We conclude that the non-equilibrium model of Gobrecht et al. (2016) is in good quantitative agreement with observational constraints on the gas-phase titanium species in Mira. The observations are in stark contrast to the older models that assumed chemical equilibrium. The most apparent difference is the almost complete oxidation of $\mathrm{TiO}$ to $\mathrm{TiO}_{2}$ at temperatures above $1000 \mathrm{~K}$ in the models (Gail \& Sedlmayr 1998; Sharp \& Huebner 1990) whereas observations indicate that both species are abundant at much lower temperatures.

\subsection{Implications for seed formation}

On the assumption that $\mathrm{TiO}_{2}$ provides the first condensation nuclei in the heterogeneous formation of inorganic dust, we would expect that the first solid titanium compounds form at very high temperatures, $1000-1500 \mathrm{~K}$. Also, taking the low elemental abundance of $\mathrm{Ti}$, the nucleation would have to be very efficient to provide enough condensation cores for formation of silicates at the observed rates. In this section, we confront these expectations with the observed characteristics of gas-phase Ti-bearing species in Mira. 
It is often assumed that titanium seeds would consist of solid $\mathrm{TiO}_{2}$ (titania) or/and its clusters and to a lesser degree ${ }^{6}$ of solid TiO. Solid $\mathrm{TiO}_{2}$ forms mainly from gas-phase $\mathrm{TiO}$ and solid $\mathrm{TiO}$ forms from gaseous $\mathrm{TiO}_{2}$ (Gail \& Sedlmayr 1998). Formation of both types of solids therefore requires an efficient formation of the simple gas-phase oxides. Our observations indicate that most of the Ti-bearing molecular gas resides in regions of the extended atmosphere of temperatures of 170 and $500 \mathrm{~K}$ (Sect. 5.5), i.e., regions where we could expect most efficient formation of the titanium solids owing to this high density of gas-phase oxides. If this condensation indeed took place therein, the dust temperatures would be low, definitely much below $1000 \mathrm{~K}$. At these temperatures, however, silicates form easily (Jeong et al. 2003; Goumans \& Bromley 2013; Gobrecht et al. 2016) and there is no need to introduce titanium oxides to initiate silicate nucleation (Gail \& Sedlmayr 1998; Jeong et al. 2003). Because we find no traces of hot titanium oxides in the circumstellar environment of Mira, the formation of hot titanium condensates is very unlikely.

Our observations show that nearly all titanium is present in the gas phase in atomic and oxidized forms. There is a chance that some amount of $\mathrm{Ti}$ is locked into solid compounds because the derived abundances for $\mathrm{TiO}$ and $\mathrm{TiO}_{2}$ are uncertain by more than one order of magnitude, but we think this is unlikely. The $\mathrm{TiO}_{2}$ to $\mathrm{TiO}$ abundance ratio of $30 \%$ indicates that the production of gaseous $\mathrm{TiO}_{2}$ is inefficient. One can expect that the transition to solids also has a limited efficiency and in consequence any solids, if formed, would have an abundance that is a small fraction of the elemental abundance of Ti. Because the cosmic abundance of $\mathrm{Ti}$ is very low compared to other metals, a high nucleation degree would be necessary to consider it a key player in dust formation. It is therefore apparent that the amount of solid titanium oxides is inadequate to create sufficient nucleation seeds.

Although we have shown that solid $\mathrm{TiO}_{2}$ does not form close to the star at high temperatures, we consider a formation of homo-molecular condensates of $\mathrm{TiO}_{2}$ at larger distances from the star, where temperatures are a few hundred K. The lowtemperature environment would pose strong constraints on the forms of solid $\mathrm{TiO}_{2}$. The crystalline form, rutile, can form from anatase and brookite after annealing in temperatures higher than $1188 \mathrm{~K}$ and $1023 \mathrm{~K}$, respectively (Brandes and Brook 1992). Therefore, one does not expect to observe rutile in O-rich AGB stars. This can be verified in the future through infrared spectroscopy, although solid titanium compounds have not been conclusively identified in spectra of AGB stars (Sect. 1).

Even if titanium oxides are not dominant in the formation of first hot monomolecular condensates, titanium can still play a positive role in dust formation in evolved stars. The low abundance of gas-phase $\mathrm{Ti}$ in the interstellar medium is the strongest argument that Ti is depleted into dust (Churchwell et al. 1980). Titanium can mix with more common oxides, helping to make the dust-formation process more rapid and efficient. Goumans \& Bromley (2013) proposed, for instance, an association reaction of $\mathrm{TiO}_{2}$ and $\mathrm{SiO}$ resulting in $\mathrm{TiSiO}_{3}$, which could be another potential source of first condensates. This and similar reactions may eventually lead to incorporation of $\mathrm{Ti}$ into dust. Another argument in support of the positive role of Ti in dust formation comes from studies of presolar grains. Titanium was found in a corundum (crystalline $\mathrm{Al}_{2} \mathrm{O}_{3}$ ) grain originating from an AGB star and it was proposed that, similarly to what is seen in synthetic $\mathrm{Al}_{2} \mathrm{O}_{3}$, trace amounts of Ti can stabilize the crystalline structure of corundum (Stroud et al. 2004).

At TE, solid TiO does not form (Gail \& Sedlmayr 1998).
A plausible alternative channel of heterogeneous formation of inorganic dust might start with alumina instead of titanium seeds. An attempt to test the role of alumina in dust formation was presented in Paper I. The direct precursor of alumina dust, $\mathrm{Al}_{2} \mathrm{O}_{3}$, is gaseous $\mathrm{AlO}$ (Gobrecht et al. 2016). Its location within the extended atmosphere $\left(\lesssim 4 R_{\star}\right)$, excitation temperature $(330 \mathrm{~K})$, and abundance relative to hydrogen $\left(10^{-9}-10^{-7}\right)$ are very similar to those derived for $\mathrm{TiO}$. The source-averaged column density ratio of $\mathrm{TiO}$ to $\mathrm{AlO}$ is $\sim 8$. Although this ratio may change considerably within the inhomogeneous atmosphere, it is surprising that $\mathrm{Al}$ gas, whose elemental abundance is 35 times higher than that of $\mathrm{Ti}$, produces a much smaller abundance of monoxide molecules. Because $\mathrm{AlO}$ was observed at five times better angular resolution than $\mathrm{TiO}$, part of the discrepancy may arise from an inaccurate determination of the size of the $\mathrm{TiO}$ emission region. A more tempting interpretation of this high ratio is that the column density of AlO is low owing to its efficient depletion into dust. All observations presented in Paper I are in favor of this, but qualitative determination of the depletion rates have not been possible. Considering all the material and discussions in this series of papers, so far $\mathrm{Al}_{2} \mathrm{O}_{3}$ appears to be the most likely condensate that initiates the dust-formation sequence in O-rich stars.

An important question is whether our findings on the lack of Ti seeds in Mira can be generalized and applied to the entire family of O-rich AGB stars or even all O-rich evolved stars of different masses. Although $o$ Ceti is one of the closest AGB stars and in many aspects is prototypical, it was chosen to be the main target of our study owing to relatively easily accessible observations covering several wavelength regimes and multiple epochs. The circumstellar chemistry leading to dust formation can be strongly influenced by parameters such as the shock strength, the pulsation period, the mass-loss rate, effective temperature, and metallicity of the star. As mentioned in Paper I (Sect. 1.3 therein), different types of O-rich AGB stars were identified through their mid-infrared spectra, including a group rich in silicates (of which Mira belongs) and those that appear to be dominated by alumina dust (Lorenz-Martins \& Pompeia 2000; Little-Marenin \& Little 1990). One can therefore expect different channels for formation, growth, and processing of grains around these stars and a large sample of objects should be studied to fully explore the various possibilities. Nevertheless, the insufficient formation of Ti seeds concluded here is in accord with findings of a similar study of the red supergiant VY CMa (Kamiński et al. 2013; De Beck et al. 2015). The characteristics of Ti-bearing gas-phases species in Mira and VY CMa are very similar although many parameters of the stars and their envelopes are different; for example, the mass-loss rate of Mira is three orders of magnitude lower than that of VY CMa. The combined results of these studies suggest that titanium oxides are an unlikely source of the first hot condensates in a broad range of circumstellar environments.

\section{3. $\mathrm{Ti}$ isotopes and s-processes in AGB stars}

Results in Table 3 and in Sect. 6.3 put constraints on the isotopic ratios of the five stable isotopes of Ti in Mira. Although the rare isotopologues were observed mainly to aid our excitation analysis, in general they offer the possibility to study nucleosynthesis in AGB stars. The Ti isotopic ratios in Mira are consistent with the solar isotopic composition but our observations and analysis methods allowed an accuracy of only a few tens of $\%$, which may be too low to trace subtle nucleosynthesis effects such as those expected from $s$-processes in AGB stars. 
Because the mass, age, and internal structure of Mira are not well known, it is difficult to predict whether there are any mechanisms that would lead to nonsolar isotopic ratios of Ti in this particular star. Some presolar grains thought to originate from (carbonrich) AGB stars show deviations from solar isotopic Ti composition as high as $\sim 40 \%$ (e.g., Hoppe et al. 1994). The material presented here illustrates that the sensitivity of ALMA allows for measurements of $\mathrm{Ti}$ isotopic ratios at a much better accuracy than has been possible in the optical and dedicated ALMA observations can deliver isotopic ratios that could be useful in studying nucleosynthesis in AGB stars.

\subsection{Conclusions}

The gas-phase chemistry of titanium in the photosphere and atmosphere of Mira is dominated by $\mathrm{Ti}$ I, TiO, and $\mathrm{TiO}_{2}$. The observed characteristics of the gas-phase Ti-bearing species rule out chemical models of the circumstellar gas that is in thermal equilibrium but they support the more realistic models that take shocks in the atmosphere into account. We do not find traces of hot circumstellar Ti-bearing gas, which makes the presence of hot titanium solids very unlikely. Also, the abundance of the gas-phase species is too high for substantial amounts of titanium locked in solids. Although titanium oxides are unlikely to produce the seeds that initiate dust nucleation in warm envelopes of O-rich AGB stars, titanium might still participate and support production of silicate dust in these stars. In conjunction with the results presented in Paper I, we consider alumina dust to be a better candidate for the seed nuclei.

Acknowledgements. We thank Hans von Winckel for making available to us the Mercator spectra of Mira. We also thank our referee for comments that helped to improve presentation of the results. Based on observations made with the Mercator Telescope, operated on the island of La Palma by the Flemmish Community, at the Spanish Observatorio del Roque de los Muchachos of the Instituto de Astrofísica de Canarias. Based on observations obtained with the HERMES spectrograph, which is supported by the Research Foundation - Flanders (FWO), Belgium, the Research Council of KU Leuven, Belgium, the Fonds National de la Recherche Scientifique (F.R.S.-FNRS), Belgium, the Royal Observatory of Belgium, the Observatoire de Genève, Switzerland, and the Thüringer Landessternwarte Tautenburg, Germany. We acknowledge with thanks the variable star observations from the AAVSO International Database contributed by observers worldwide and used in this research. Based on data obtained from the ESO Science Archive Facility and made with ESO Telescopes at APEX and the La Silla and Paranal Observatories under program IDs 074.D-0114(A), 089.D-0383(A), 097.D-0414(A), and 097.D-0414(B). Based on observations obtained at the Canada-France-Hawaii Telescope (CFHT) which is operated by the National Research Council of Canada, the Institut National des Sciences de l'Univers of the Centre National de la Recherche Scientique of France, and the University of Hawaii. Herschel is an ESA space observatory with science instruments provided by European-led Principal Investigator consortia and with important participation from NASA. Based on analysis carried out with the CASSIS software developed by IRAP-UPS/CNRS and CDMS and JPL spectroscopic databases. This paper makes use of the following ALMA data: ADS/JAO.ALMA\#2011.0.00014.SV, 2013.1.00047.S 2012.1.00524.S, 2013.1.00156.S. ALMA is a partnership of ESO (representing its member states), NSF (USA) and NINS (Japan), together with NRC (Canada), NSC and ASIAA (Taiwan), and KASI (Republic of Korea), in cooperation with the Republic of Chile. The Joint ALMA Observatory is operated by ESO, AUI/NRAO, and NAOJ.

\section{References}

Berglund, M., \& Wieser, M. E. 2011, Pure Appl. Chem., 83, 397

Brandes, E. A., \& Brook, G. B. 1992, Smithells Metals Reference Book (Oxford: Butterworth-Heinemann Ltd.)

Brünken, S., Müller, H. S. P., Menten, K. M., McCarthy, M. C., \& Thaddeus, P. 2008, ApJ, 676, 1367

Bromley, S. T., Gómez Martín, J. C. \& Plane, J. M. C. 2016, Phys. Chem. Chem. Phys., 18, 26913
Burrows, A., \& Sharp, C. M. 1999, ApJ, 512, 843

Burrows, A., Dulick, M., Bauschlicher, C. W., Jr., et al. 2005, ApJ, 624, 988

Chavez, J., \& Lambert, D. L. 2009, ApJ, 699, 1906

Cherchneff, I. 2006, A\&A, 456, 1001

Cherchneff, I. 2012, A\&A, 545, A12

Churchwell, E., Hocking, W. H., Merer, A. J., \& Gerry, M. C. L. 1980, AJ, 85, 1382

Clayton, D. 2003, Handbook of Isotopes in the Cosmos, Cambridge (UK: Cambridge Univ. Press)

Danchi, W. C., Bester, M., Degiacomi, C. G., Greenhill, L. J., \& Townes, C. H 1994, AJ, 107, 1469

De Beck, E., Vlemmings, W., Muller, S., et al. 2015, A\&A, 580, A36

Duari, D., Cherchneff, I., \& Willacy, K. 1999, A\&A, 341, L47

Fabas, N., Lèbre, A., \& Gillet, D. 2011, A\&A, 535, A12

Ferrarotti, A. S., \& Gail, H.-P. 2006, A\&A, 447, 553

Ferlet, R., \& Gillet, D. 1984, A\&A, 133, L1

ALMA Partnership, Fomalont, E. B., Vlahakis, C., et al. 2015, ApJ, 808, L1

Forsberg, P. 1991, Phys. Scr., 44, 446

Fowler, A. 1909, MNRAS, 69, 508

Fox, M. W., Wood, P. R., \& Dopita, M. A. 1984, ApJ, 286, 337

Gail, H.-P., \& Sedlmayr, E. 1986, A\&A, 166, 225

Gail, H.-P., \& Sedlmayr, E. 1998, Faraday Discussions, 109, 303

Gail, H.-P., Zhukovska, S. V., Hoppe, P., \& Trieloff, M. 2009, ApJ, 698, 1136

Gail, H.-P., Wetzel, S., Pucci, A., \& Tamanai, A. 2013, A\&A, 555, A119

Gail, H.-P., Scholz, M., \& Pucci, A. 2016, A\&A, 591, A17

Gehrz, R. 1989, Interstellar Dust, 135, 445

Gendriesch, R., Lewen, F., Klapper, G., et al. 2009, A\&A, 497, 927

Gobrecht, D., Cherchneff, I., Sarangi, A., Plane, J. M. C., \& Bromley, S. T. 2016, A\&A, 585, A6

Goldsmith, P. F., \& Langer, W. D. 1999, ApJ, 517, 209

Goumans, T. P. M., \& Bromley, S. T. 2013, Phil. Trans. R. Soc. A, 371, 20110580

Gustafsson, B., Edvardsson, B., Eriksson, K., et al. 2008, A\&A, 486, 951

Haniff, C. A., Ghez, A. M., Gorham, P. W., et al. 1992, AJ, 103, 1662

Hinkle, K. H. 1978, ApJ, 220, 210

Hinkle, K. H., Scharlach, W. W. G., \& Hall, D. N. B. 1984, ApJS, 56, 1

Howard, W. M., Arnett, W. D., Clayton, D. D., \& Woosley, S. E. 1972, ApJ, 175, 201

Höfner, S., Bladh, S., Aringer, B., \& Ahuja, R. 2016, A\&A, 594, A108

Hoppe, P., Amari, S., Zinner, E., Ireland, T., \& Lewis, R. S. 1994, ApJ, 430, 870

Ireland, M. J., Scholz, M., \& Wood, P. R. 2011, MNRAS, 418, 114

Jeong, K. S., Winters, J. M., \& Sedlmayr, E. 1999, Asymptotic Giant Branch Stars, 191, 233

Jeong, K. S., Winters, J. M., Le Bertre, T., \& Sedlmayr, E. 2003, A\&A, 407, 191

Jonsson, J., Launila, O., \& Lindgren, B. 1992, MNRAS, 258, 49

Kamiński, T., Gottlieb, C. A., Menten, K. M., et al. 2013, A\&A, 551, A113

Kamiński, T., Wong, K. T., Schmidt, M. R., et al. 2016, A\&A, 592, A42 (Paper I)

Kania, P., Hermanns, M., Brünken, S., Müller, H. S. P., \& Giesen, T. F. 2008, contribution RB12, 62nd Int. Symp. Mol. Spec., June 16-20, Columbus, Ohio, USA; https://molspect.chemistry.ohio-state. edu/symposium_63/symposium/Abstracts/p396.pdf

Kania, P., Giesen, T. F., Müller, H. S. P., Schlemmer S., \& Brünken, S. 2011, J. Mol. Spectrosc., 268, 173

Karovicova, I., Wittkowski, M., Ohnaka, K., et al. 2013, A\&A, 560, A75

Karovska, M., Hack, W., Raymond, J., \& Guinan, E. 1997, ApJ, 482, L175

Klein, T., Ciechanowicz, M., Leinz, Ch., et al. 2014, IEEE Trans. on Terahertz Science and Technology, 4, 588

Lambert, D. L., \& Luck, R. E. 1977, ApJ, 211, 443

Lee, G., Helling, C., Giles, H., \& Bromley, S. T. 2015, A\&A, 575, A11

Little-Marenin, I. R., \& Little, S. J. 1990, AJ, 99, 1173

Lodders, K. 2003, ApJ, 591, 1220

Lopez, B., Danchi, W. C., Bester, M., et al. 1997, ApJ, 488, 807

Lorenz-Martins, S., \& Pompeia, L. 2000, MNRAS, 315, 856

Matthews, L. D., Reid, M. J., \& Menten, K. M. 2015, ApJ, 808, 36

McMullin, J. P., Waters, B., Schiebel, D., Young, W., \& Golap, K. 2007, Astronomical Data Analysis Software and Systems XVI, 376, 127

Müller, H. S. P., \& Brünken, S. 2005, J. Mol. Spectrosc., 232, 213

Müller, H. S. P., Thorwirth, S., Roth, D. A., \& Winnewisser, G. 2001, A\&A, 370, L49

Müller, H. S. P., Schlöder, F., Stutzki, J., \& Winnewisser, G. 2005, J. Mol. Struct., 742,215

Müller, H. S. P., Brünken, S., McCarthy, M. C., \& Thaddeus, P. 2007, contribution RD02, 62nd Int. Symp. on Mol. Spec., June 18-22, Columbus, Ohio, USA; https://molspect.chemistry.ohio-state. edu/symposium_62/symposium/Abstracts/p219.pdf 
T. Kamiński et al.: Ti-bearing gas and dust in Mira

Namiki, K.-I., Saito, S., Robinson, J. S., \& Steimle, T. C. 1998, J. Mol. Spec., 191, 176

Nhung, P. T., Hoai, D. T., Diep, P. N., et al. 2016, MNRAS, 460, 673

Nittler, L. R., Alexander, C. M. O., Gallino, R., et al. 2008, ApJ, 682, 1450

Norris, B. R. M., Tuthill, P. G., Ireland, M. J., et al. 2012, Nature, 484, 220

Plane, J. M. C. 2013, Philos. Trans. R. Soc. London Ser. A, 371, 20120335

Planesas, P., Alcolea, J., \& Bachiller, R. 2016, A\&A, 586, A69

Plez, B. 1998, A\&A, 337, 495

Posch, T., Kerschbaum, F., Fabian, D., et al. 2003, ApJS, 149, 437

Pulliam, R. L., Zack, L. N., \& Ziurys, L. M. 2010, J. Mol. Spec., 264, 50

Ram, R. S., Bernath, P. F., Dulick, M., \& Wallace, L. 1999, ApJS, 122, 331

Ramstedt, S., Mohamed, S., Vlemmings, W. H. T., et al. 2014, A\&A, 570, L14

Raskin, G., van Winckel, H., Hensberge, H., et al. 2011, A\&A, 526, A69

Reid, M. J., \& Goldston, J. E. 2002, ApJ, 568, 931

Reid, M. J., \& Menten, K. M. 1997, ApJ, 476, 327

Reid, M. J., \& Menten, K. M. 2007, ApJ, 671, 2068

Richter, H., \& Wood, P. R. 2001, A\&A, 369, 1027

Richter, H., Wood, P. R., Woitke, P., Bolick, U., \& Sedlmayr, E. 2003, A\&A, 400, 319

Roelfsema, P. R., Helmich, F. P., Teyssier, D., et al. 2012, A\&A, 537, A17

Schoenberg, K. 1985, A\&AS, 62, 339

Sharp, C. M., \& Huebner, W. F. 1990, ApJS, 72, 417

Sheridan, P. M., McLamarrah, S. K., \& Ziurys, L. M. 2003, J. Chem. Phys., 119, 9496

Smolders, K., Verhoelst, T., Neyskens, P., et al. 2012, A\&A, 543, L2

Steimle, T. C., \& Virgo, W. 2003, Chem. Phys. Lett., 381, 30
Stokes, G. M. 1978, ApJS, 36, 115

Stroud, R. M., Nittler, L. R., \& Alexander, C. M. O. 2004, Science, 305, 1455

Tamanai, A., Mutschke, H., Blum, J., et al. 2009, A\&A, 501, 251

Tennyson, J., \& Yurchenko, S. N. 2012, MNRAS, 425, 21

Thorwirth, S., Müller, H. S. P., Lewen, F., et al. 2003, ApJ, 585, L163

Tielens, A. G. G. M. 1991, IAU Colloq. 126, Origin and Evolution of Interplanetary Dust, 173, 405

Tsuji, T. 1973, A\&A, 23, 411

Vassilev, V., Meledin, D., Lapkin, I., et al. 2008, A\&A, 490, 1157

Vlemmings, W. H. T., Ramstedt, S., O'Gorman, E., et al. 2015, A\&A, 577, L4

Wang, H., Steimle, T. C., Apetrei, C., \& Maier, J. P. 2009, Phys. Chem. Chem. Phys., 11, 2649

Wasserburg, G. J., Trippella, O., \& Busso, M. 2015, ApJ, 805, 7

Western, C. M. 2014, PGOPHER 8.0, a Program of Simulation Rotational Structure (University of Bristol)

Willacy, K., \& Cherchneff, I. 1998, A\&A, 330, 676

Willson, L. A. 1976, ApJ, 205, 172

Wong, K. T., Kamiński, T., Menten, K. M., \& Wyrowski, F. 2016, A\&A, 590, A127

Wyckoff, S., \& Wehinger, P. 1972, ApJ, 178, 481

Yu, S., Pearson, J. C., Drouin, B. J., et al. 2012, J. Mol. Spectr., 279, 16

Zhao-Geisler, R., Quirrenbach, A., Köhler, R., \& Lopez, B. 2012, A\&A, 545, A56

Zhuang, X., Le, A., Steimle, T. C., et al. 2010, Phys. Chem. Chem. Phys. (Incorporating Faraday Transactions), 12, 15018 


\section{Appendix A: Mercator/HERMES spectra}

Table A. 1 presents details of the archival observations obtained with HERMES at the Mercator telescope.

Table A.1. A log of Mercator/HERMES spectral observations of Mira.

\begin{tabular}{cllll}
\hline \hline Date & JD & $\begin{array}{l}\text { Exposure } \\
\text { time }(\mathrm{s})\end{array}$ & $\begin{array}{l}\text { Vis. } \\
\text { phase }\end{array}$ & $\begin{array}{l}\text { Vis } \\
\text { mag }\end{array}$ \\
\hline $2009-07-21$ & 2455033.7 & 600 & 0.63 & 9.2 \\
$2009-08-08$ & 2455051.7 & 900 & 0.68 & 9.1 \\
$2009-08-15$ & 2455058.7 & 600 & 0.70 & 9.2 \\
$2009-09-06$ & 2455080.7 & 600 & 0.77 & 8.9 \\
$2009-09-15$ & 2455089.6 & 200 & 0.79 & 8.4 \\
$2009-10-02$ & 2455106.7 & 100 & 0.85 & 8.0 \\
$2009-10-20$ & 2455124.6 & 130 & 0.90 & 5.4 \\
$2009-10-26$ & 2455131.5 & 100 & 0.92 & 4.6 \\
$2009-11-23^{a}$ & 2455159.5 & 100 & 0.00 & 3.9 \\
$2010-02-07$ & 2455235.4 & 60 & 0.23 & 5.8 \\
$2011-01-04$ & 2455566.3 & 6 & 0.23 & 5.1 \\
$2014-08-31$ & 2456900.7 & 20 & 0.23 & 5.8 \\
\hline
\end{tabular}

Notes. ${ }^{(a)}$ Instrumental artifacts above $8000 \AA$.

\section{Appendix B: Isotopic composition from optical bands of TiO}

We attempted to measure contributions of the five isotopologues of $\mathrm{TiO}$ to the optical absorption spectrum of Mira. Following Wyckoff \& Wehinger (1972) and Chavez \& Lambert (2009), we mainly analyzed the $(0,0)$ band of the $\gamma$ system. For the spectral synthesis, we used a list of lines of Plez (1998), where we replaced original wavelengths of transitions in the main branches by those measured in laboratory by Ram et al. (1999). For satellite branches, the wavelengths were computed from the spectroscopic constants provided by Ram et al. (1999), while isotopic shifts were computed using Dunham expansions of these constants. This modified line list was used to generate a synthetic spectrum from the MARCS model photosphere (Gustafsson et al. 2008) with physical parameters best representing $o$ Ceti ( solar chemical composition, $T_{\text {eff }}=3200 \mathrm{~K}, \log g=0$, microturbulence of $2 \mathrm{~km} \mathrm{~s}^{-1}$ ). The observations were represented by a spectrum acquired close to the maximum visual light, on 3 October 2009. Spectra in maximum correspond to the most compact phase of the photosphere and are represented relatively well by the static MARCS model. Even with this careful simulation procedure, the synthetic spectrum reproduces the TiO band very roughly. Owing to this imperfect simulation, we analyzed the isotopic composition by scaling the cumulative abundance of all the four rare titanium isotopologues rather than just one. The observed spectrum is consistent with the solar isotopic composition of $\mathrm{Ti}$ and any enhancement of the rare species would have to be smaller than $25 \%$. Upper limits on enhancements of individual isotopologues would be even higher. 


\section{Appendix C: Optical spectra of Mira}
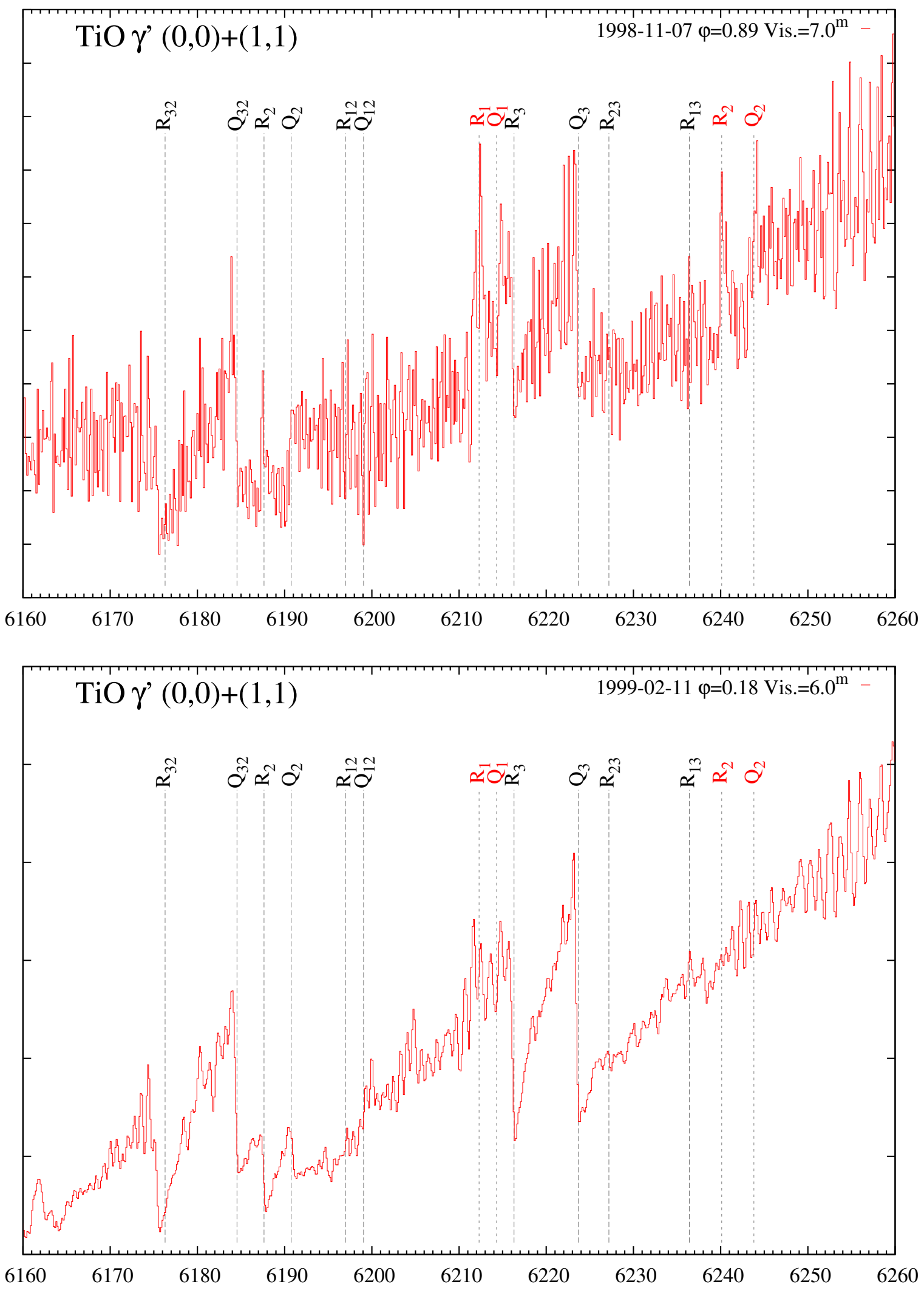

Fig. C.1. Spectra of Mira covering the part of the $\gamma^{\prime}$ system of TiO that occasionally displays emission features. The date and corresponding visual phase and magnitude are indicated in the top right corner of each panel. The spectra are shown in the chronological order. Branches forming main electronic features are indicated in black and red for the $(0,0)$ and $(1,1)$ bands, respectively. The wavelength scale in $\AA$ is in the heliocentric rest frame. 

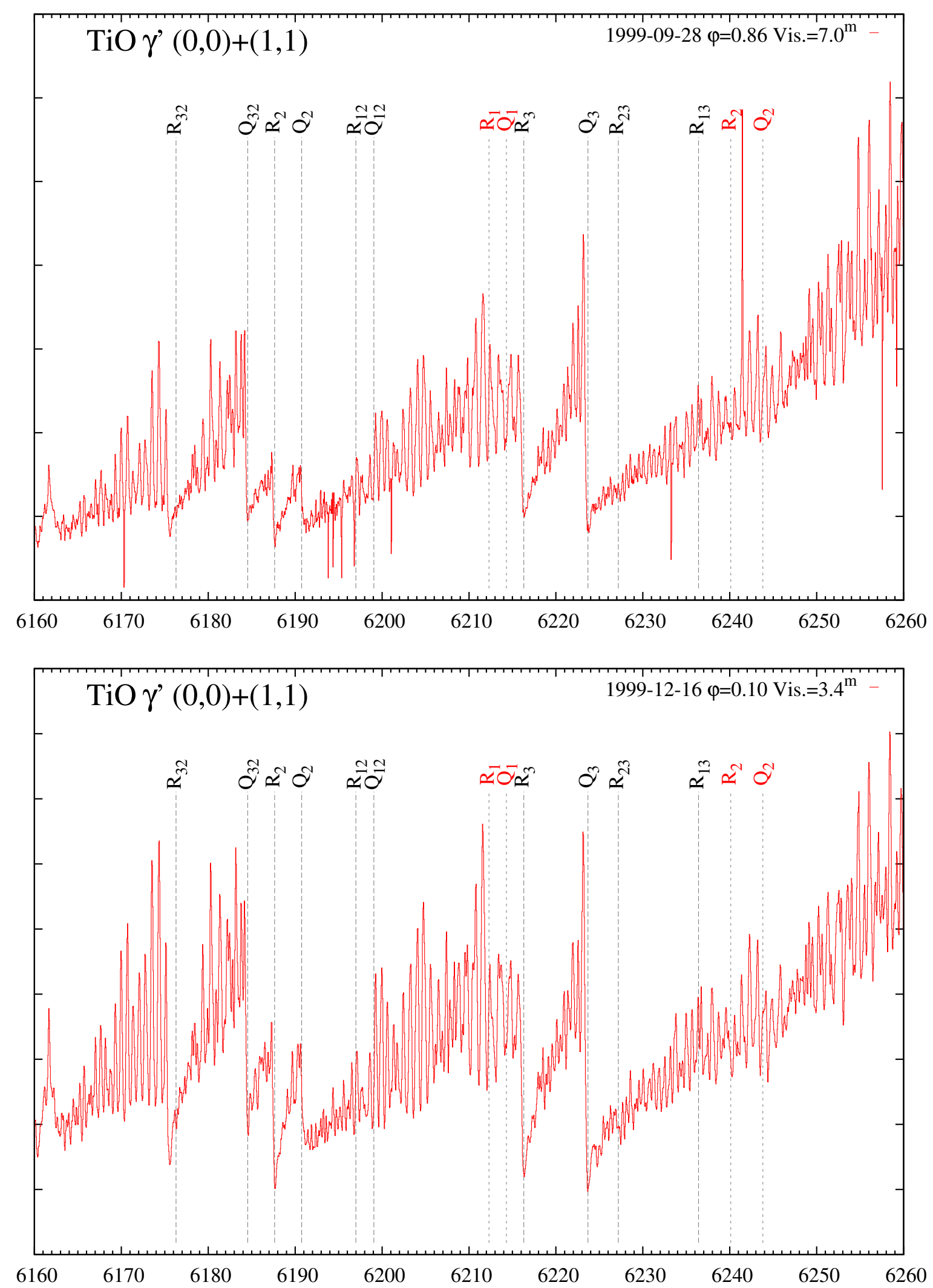

Fig. C.1. continued. 
T. Kamiński et al.: Ti-bearing gas and dust in Mira
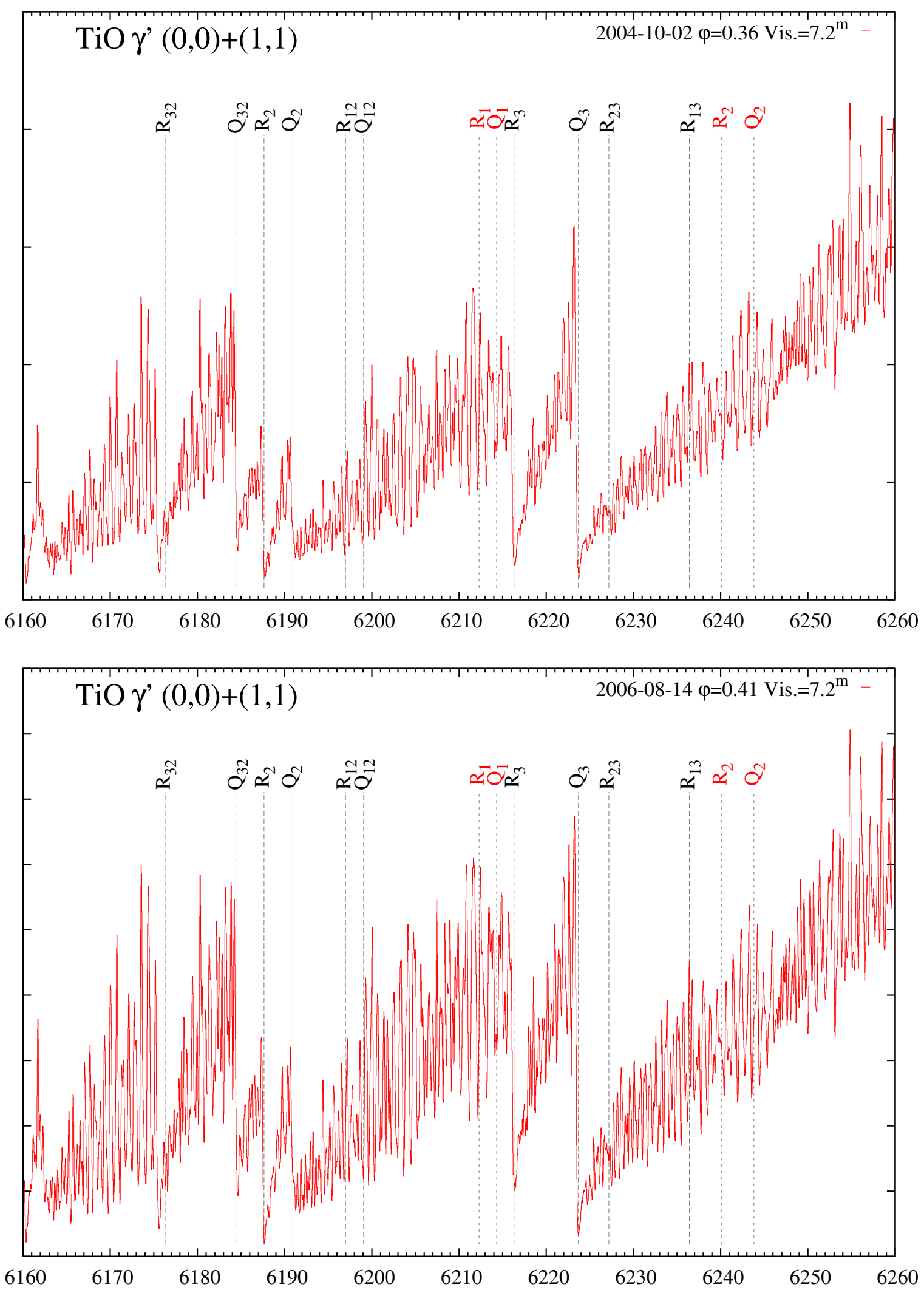

Fig. C.1. continued. 

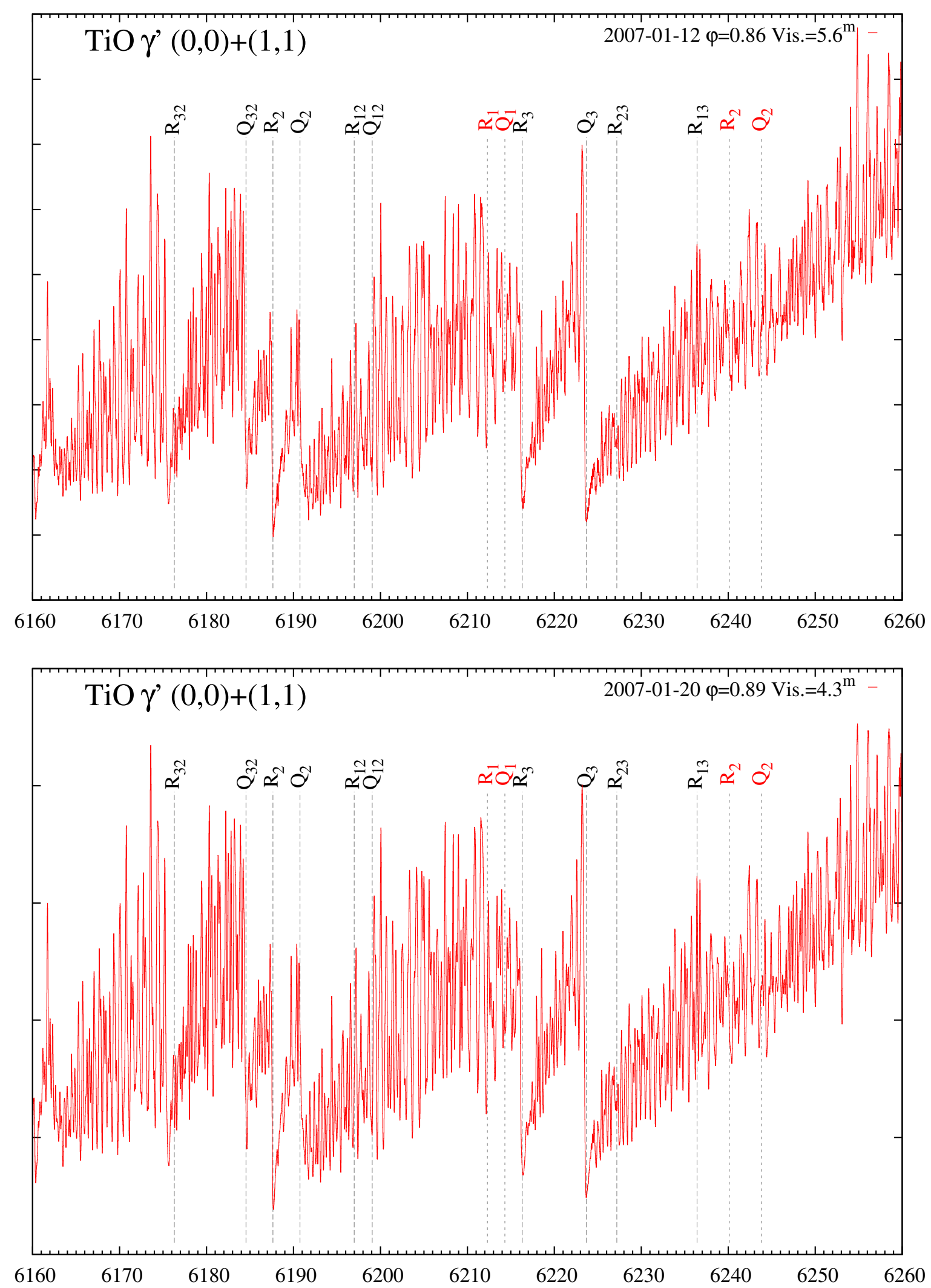

Fig. C.1. continued. 
T. Kamiński et al.: Ti-bearing gas and dust in Mira
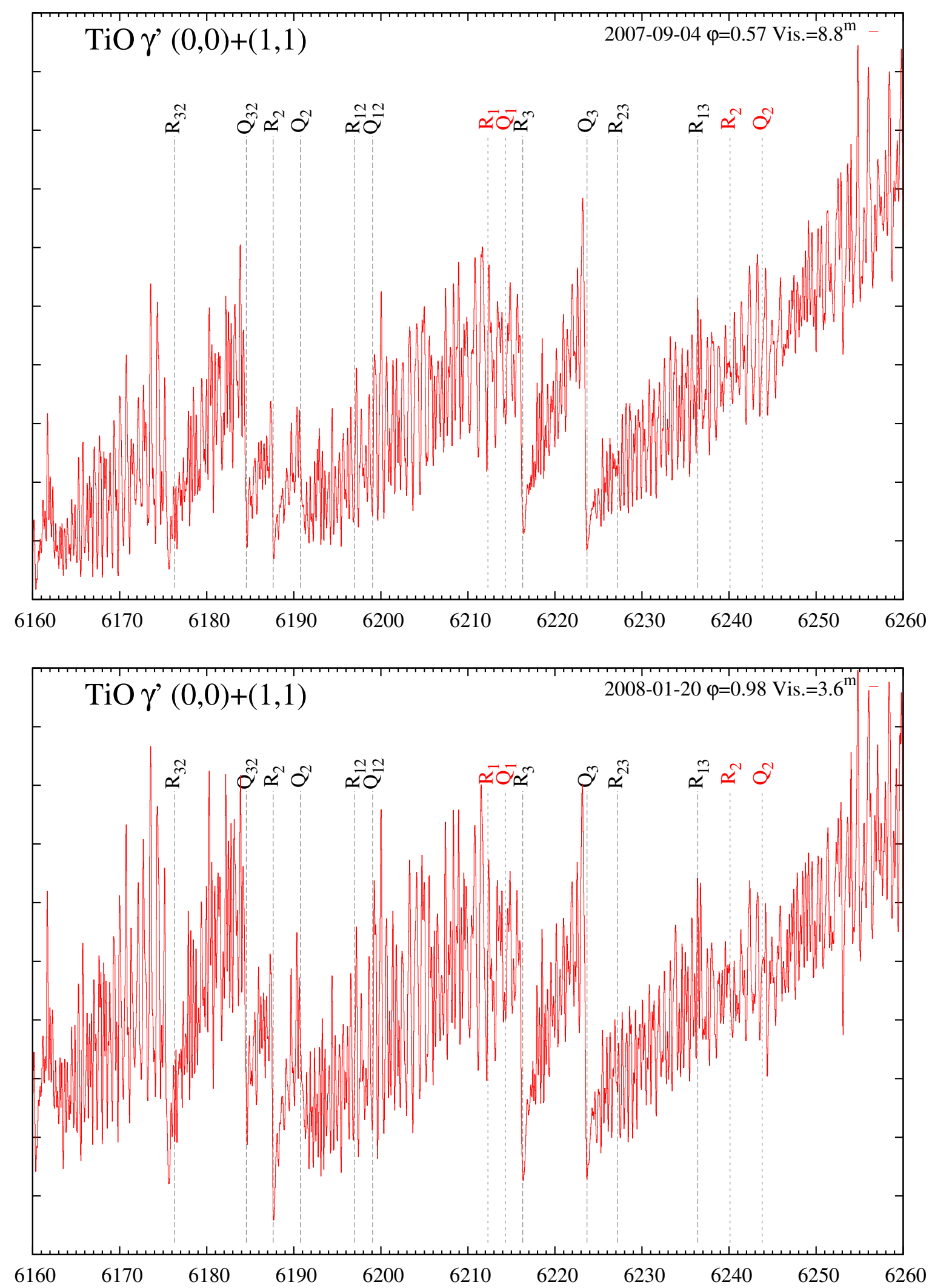

Fig. C.1. continued. 

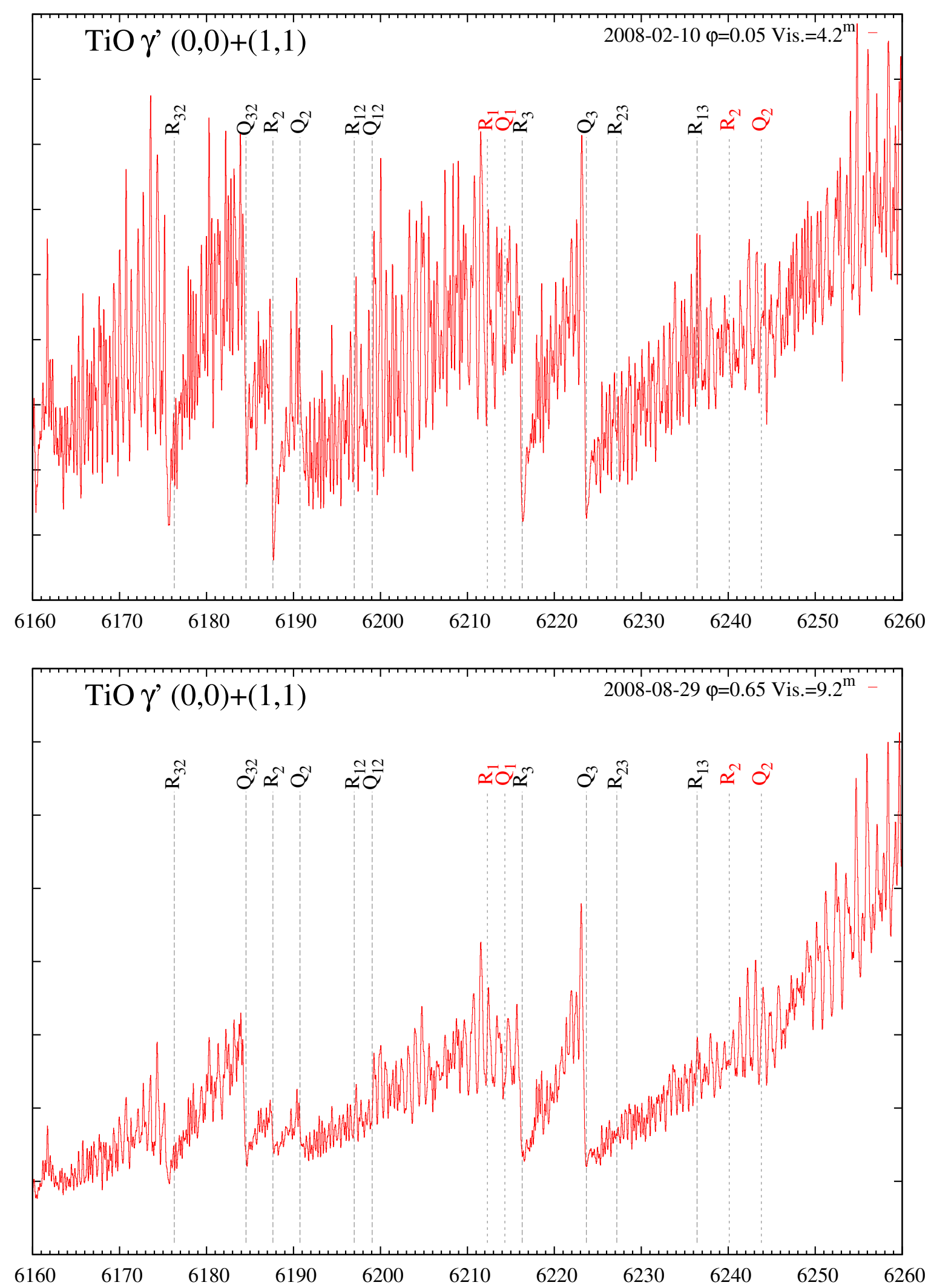

Fig. C.1. continued. 
T. Kamiński et al.: Ti-bearing gas and dust in Mira
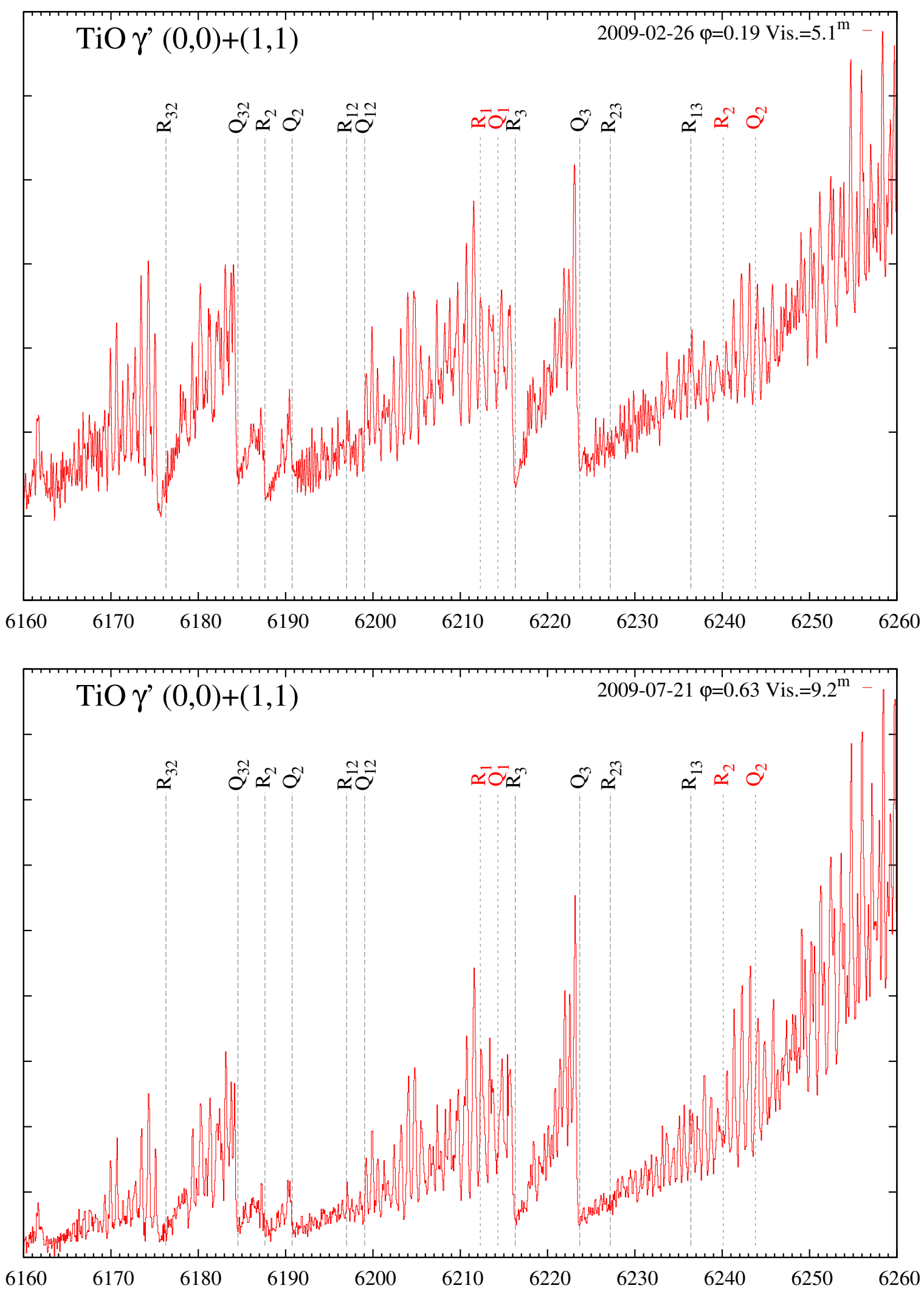

Fig. C.1. continued. 

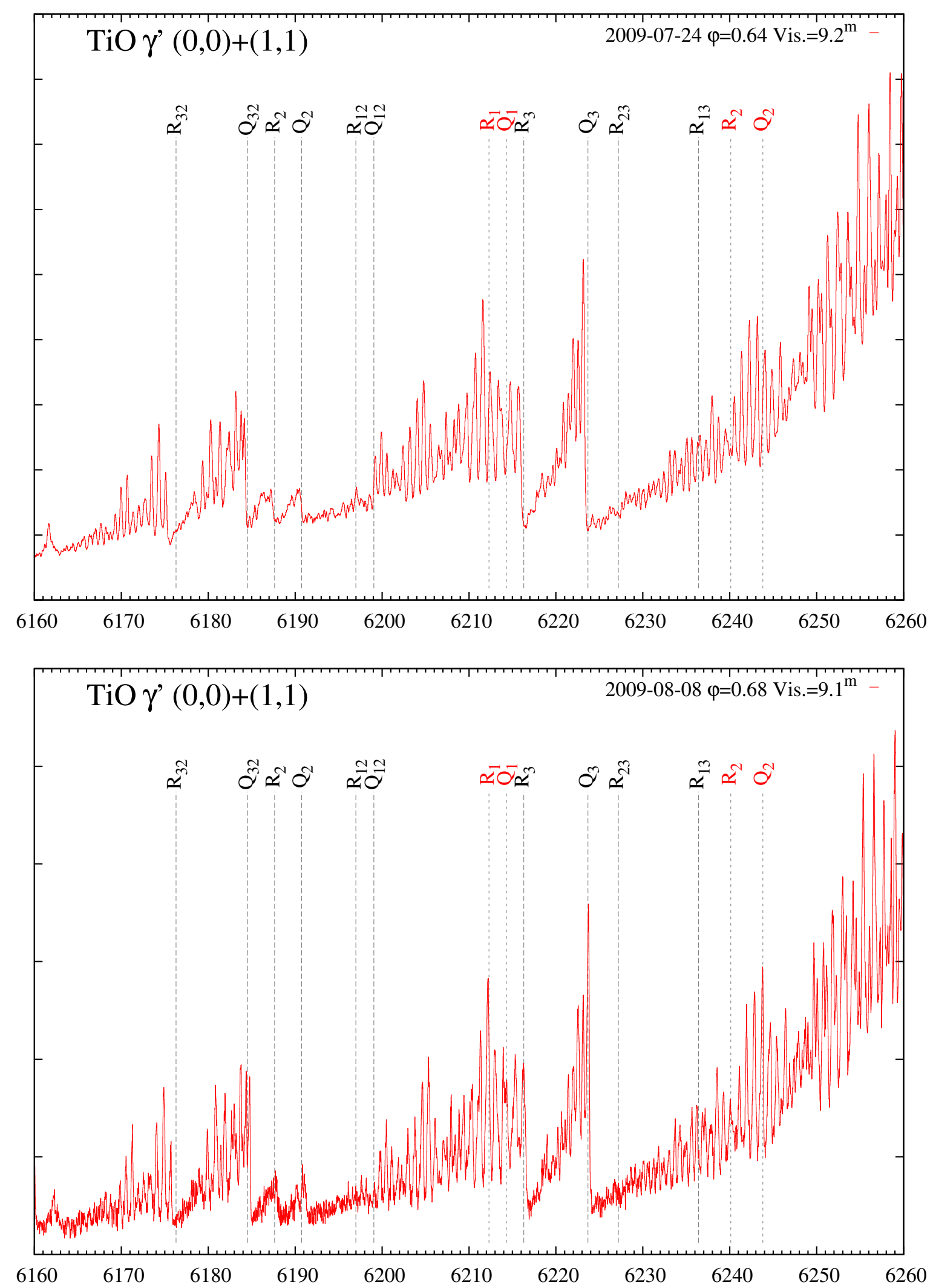

Fig. C.1. continued. 
T. Kamiński et al.: Ti-bearing gas and dust in Mira
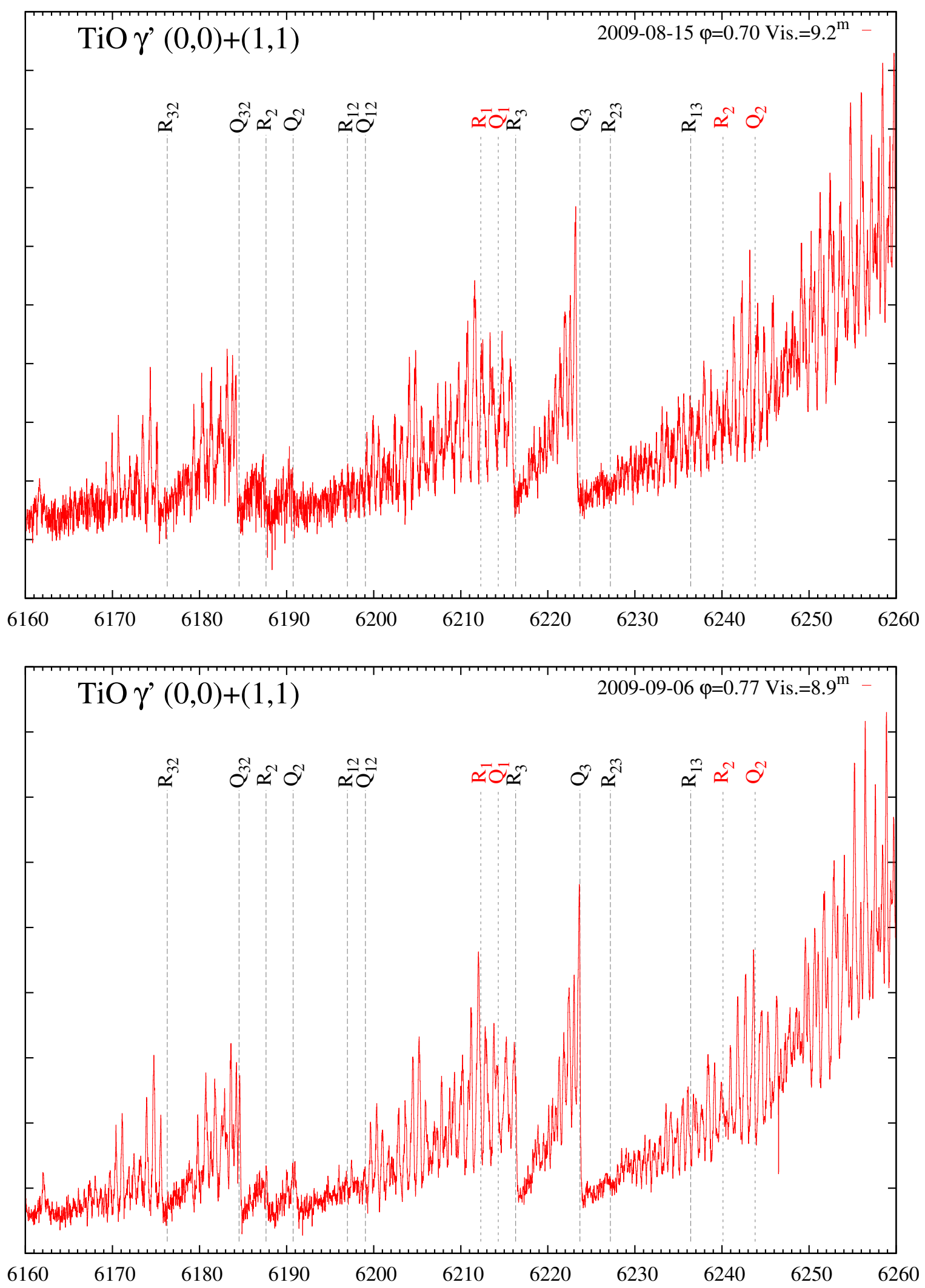

Fig. C.1. continued. 

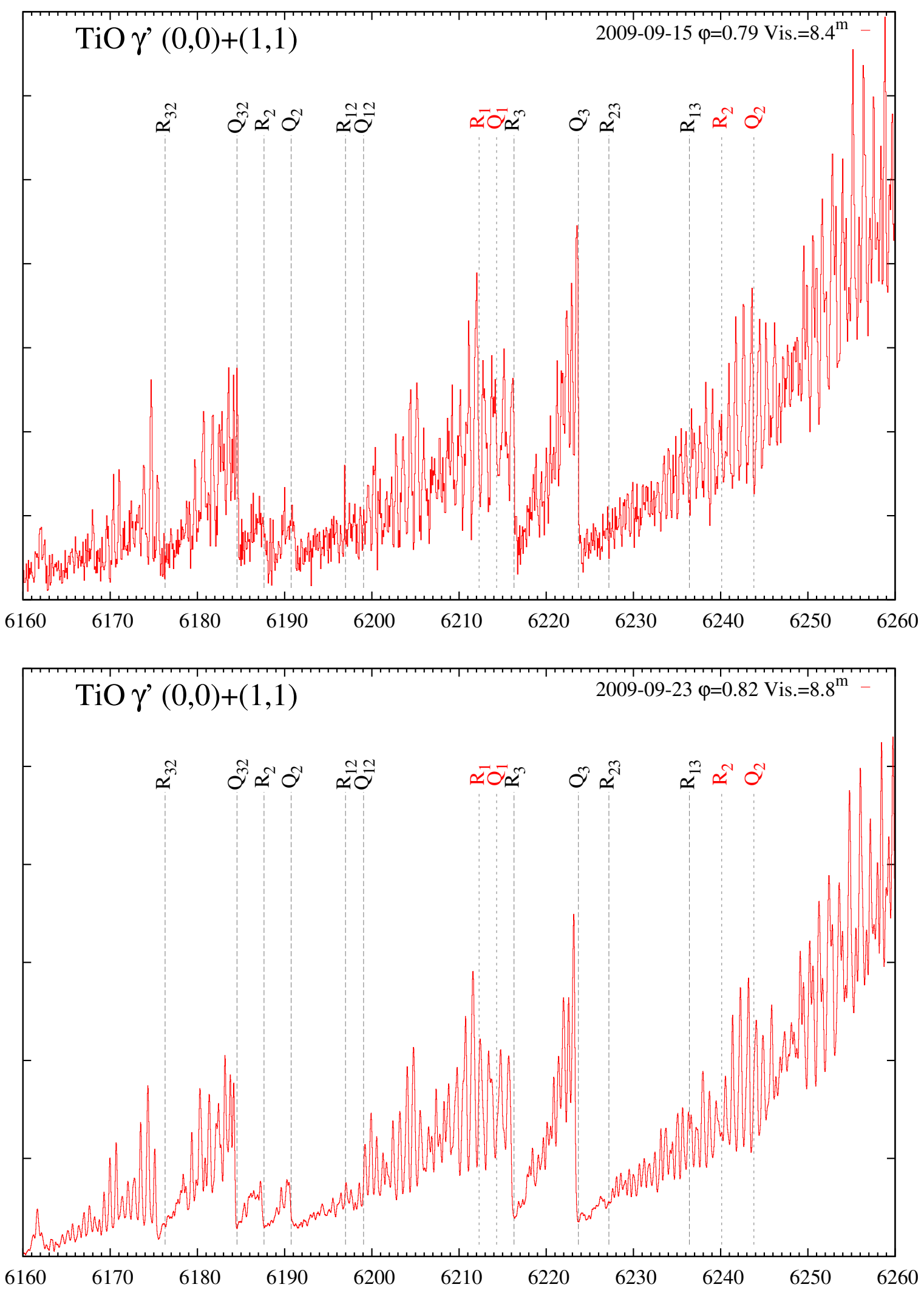

Fig. C.1. continued. 
T. Kamiński et al.: Ti-bearing gas and dust in Mira
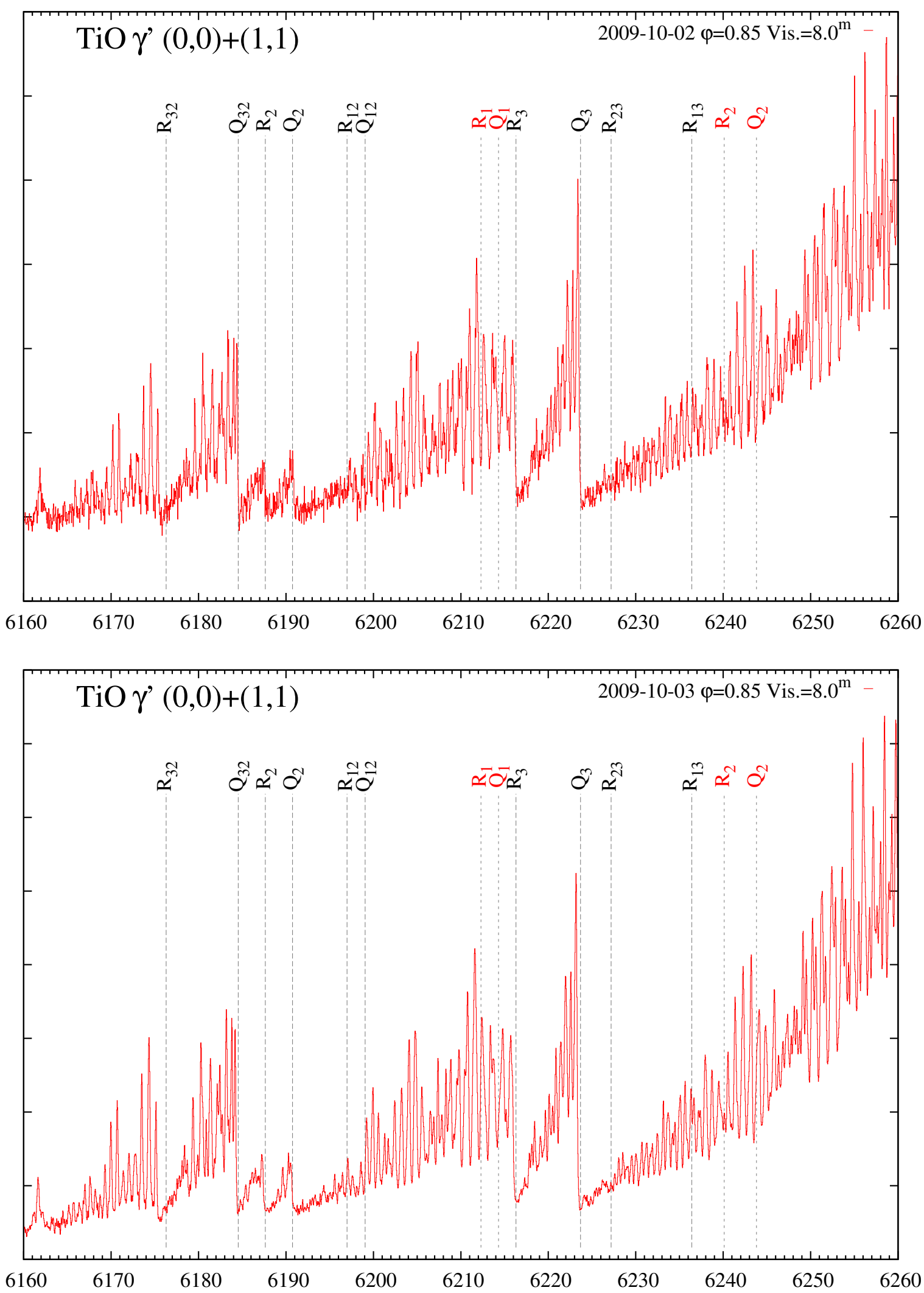

Fig. C.1. continued. 

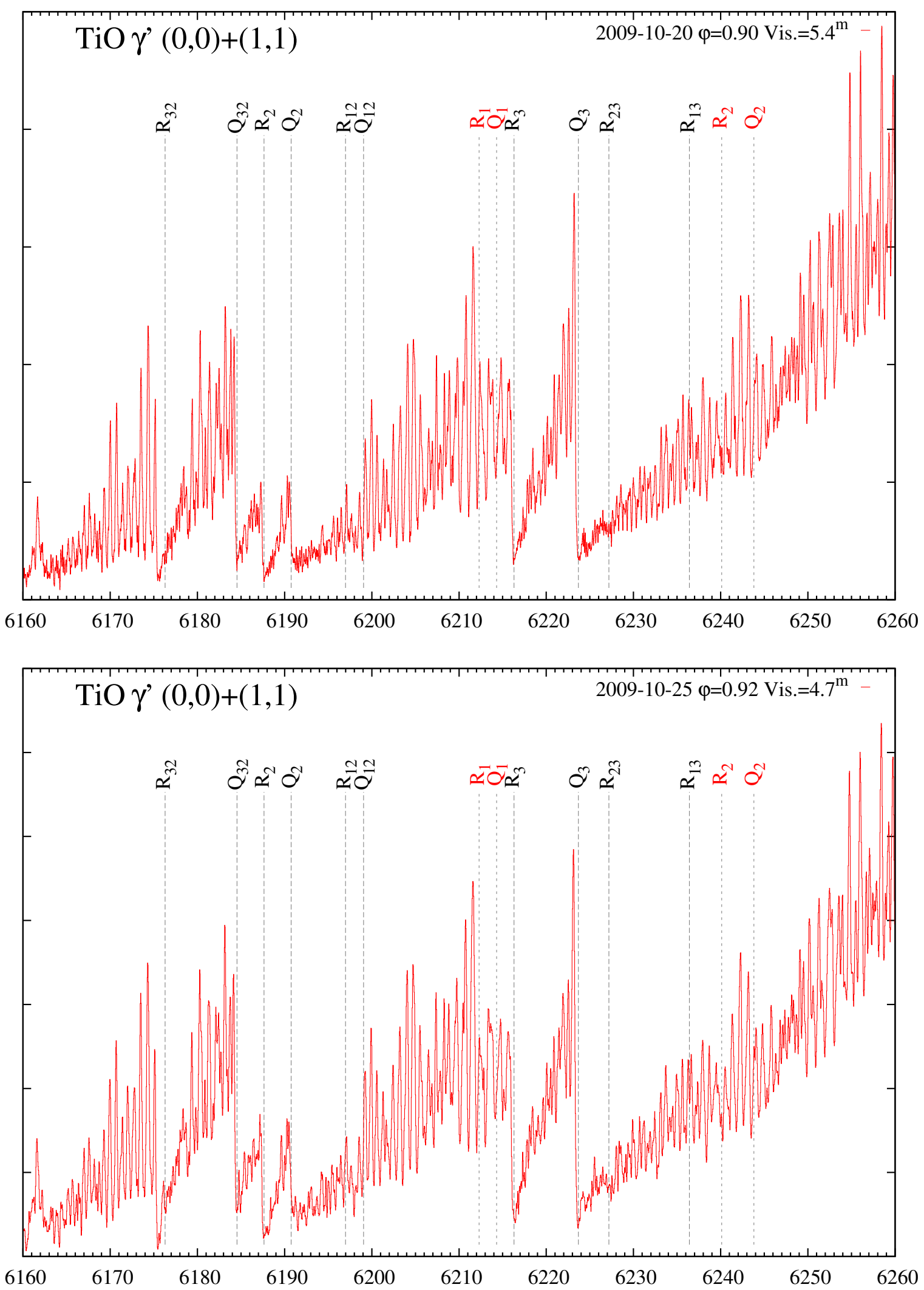

Fig. C.1. continued. 
T. Kamiński et al.: Ti-bearing gas and dust in Mira
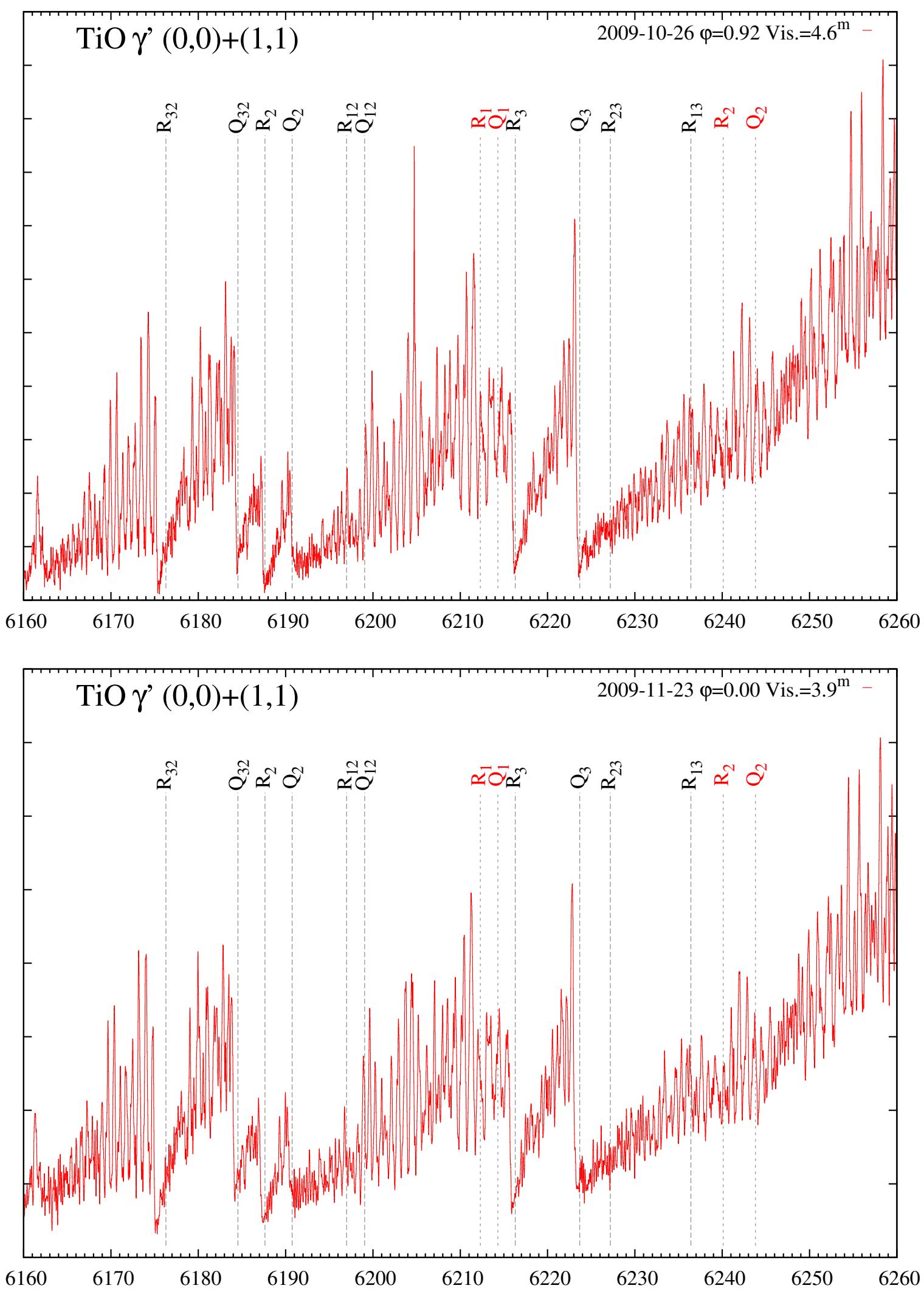

Fig. C.1. continued. 

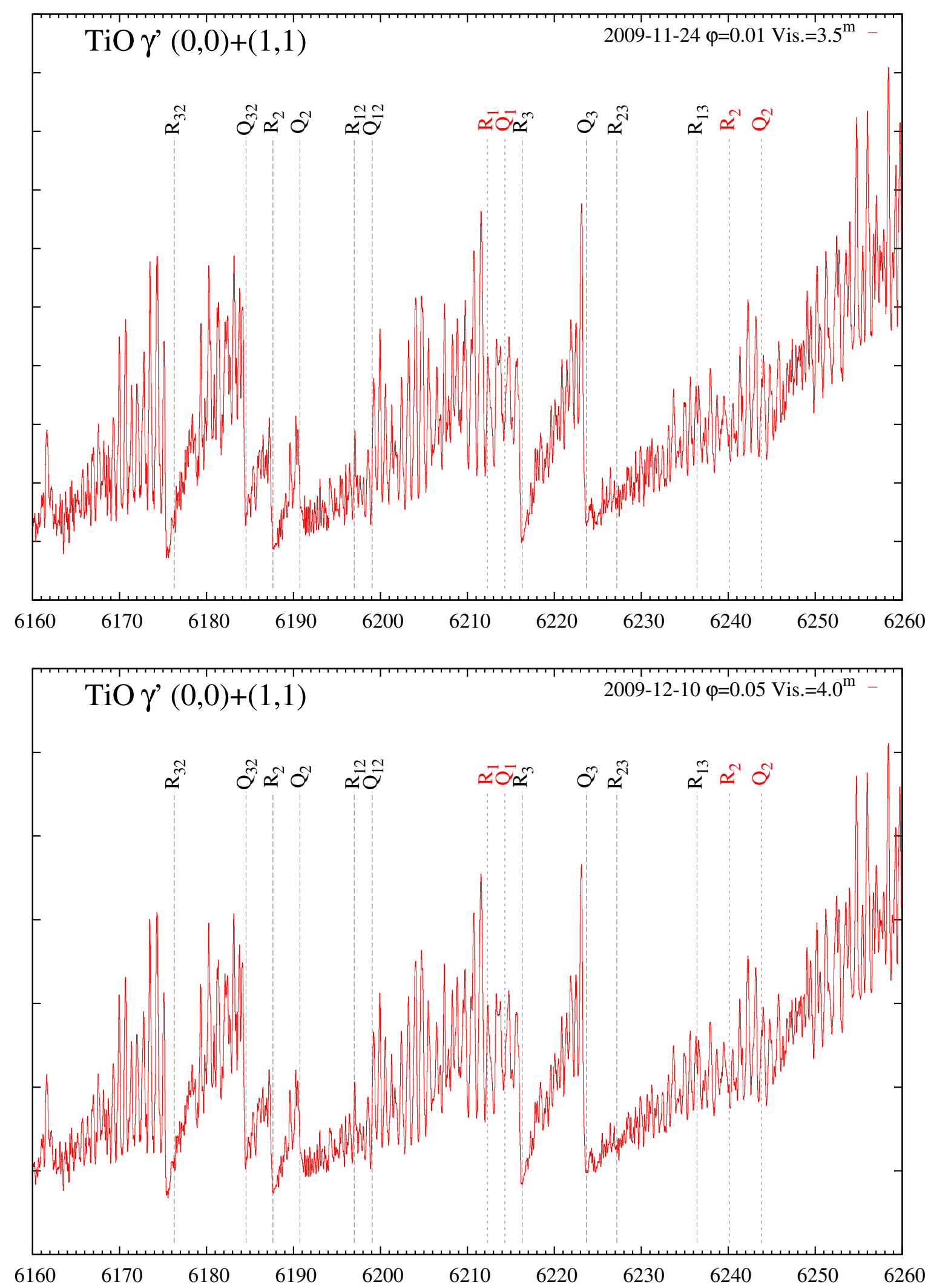

Fig. C.1. continued. 
T. Kamiński et al.: Ti-bearing gas and dust in Mira
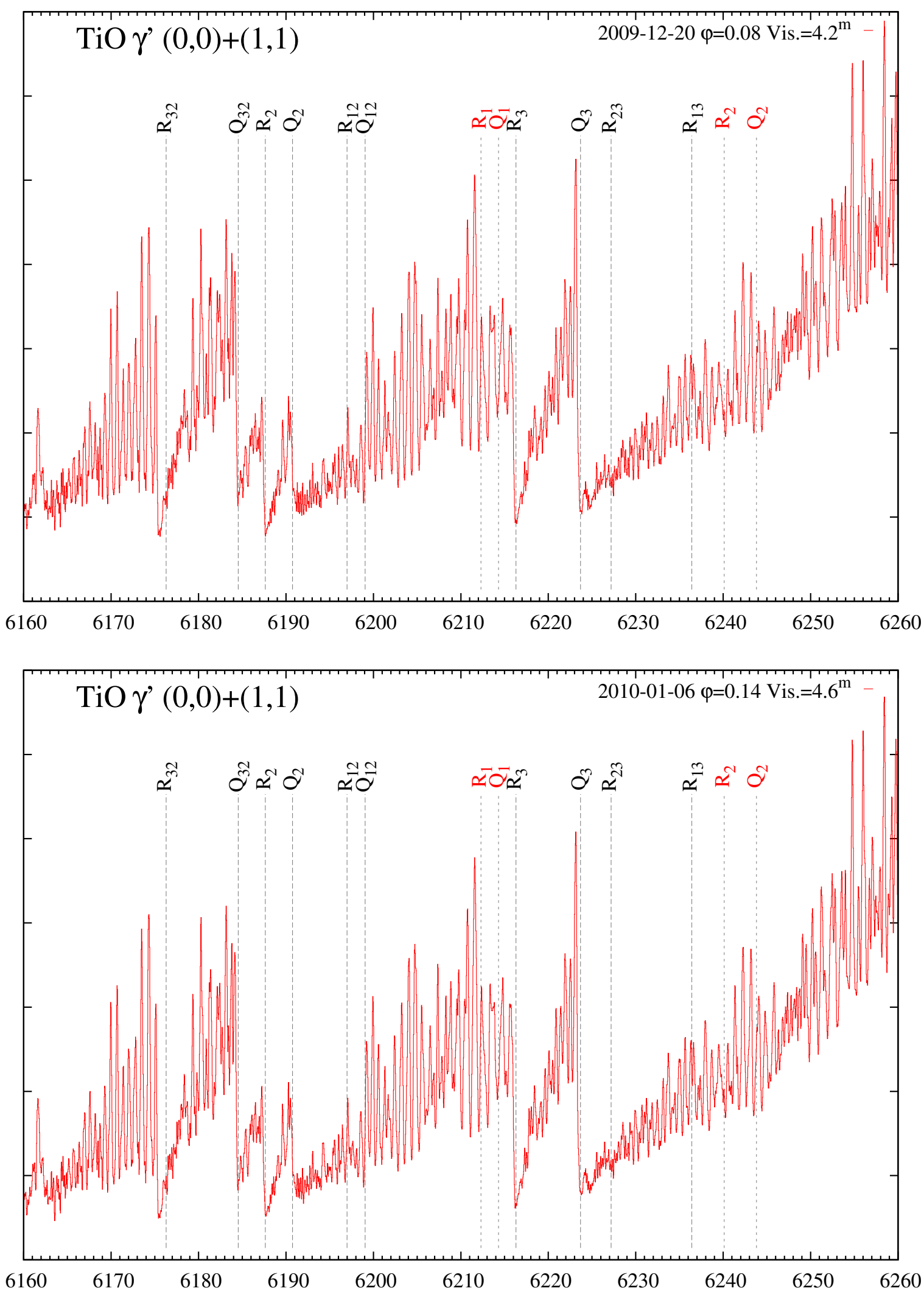

Fig. C.1. continued. 

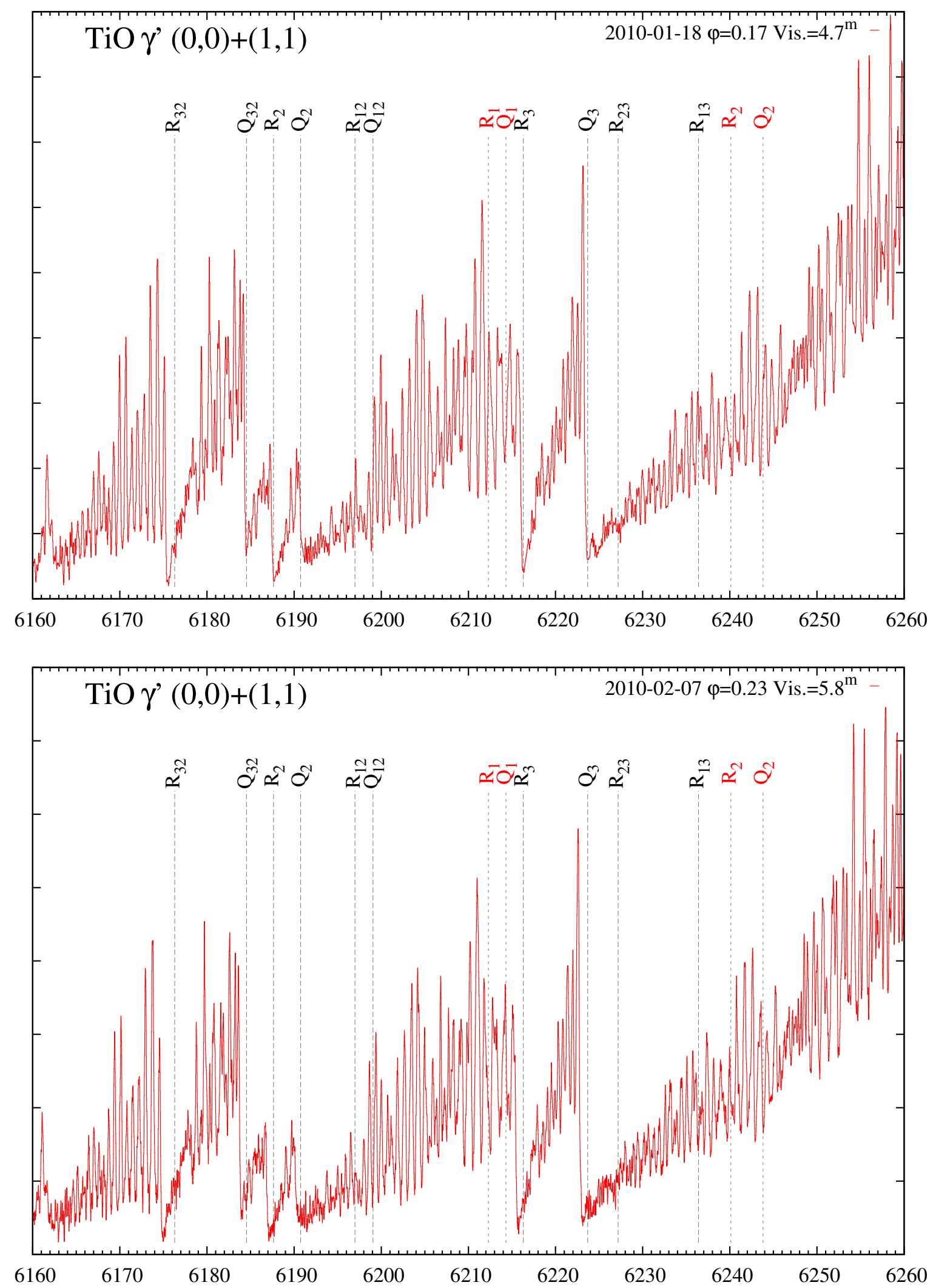

Fig. C.1. continued. 
T. Kamiński et al.: Ti-bearing gas and dust in Mira
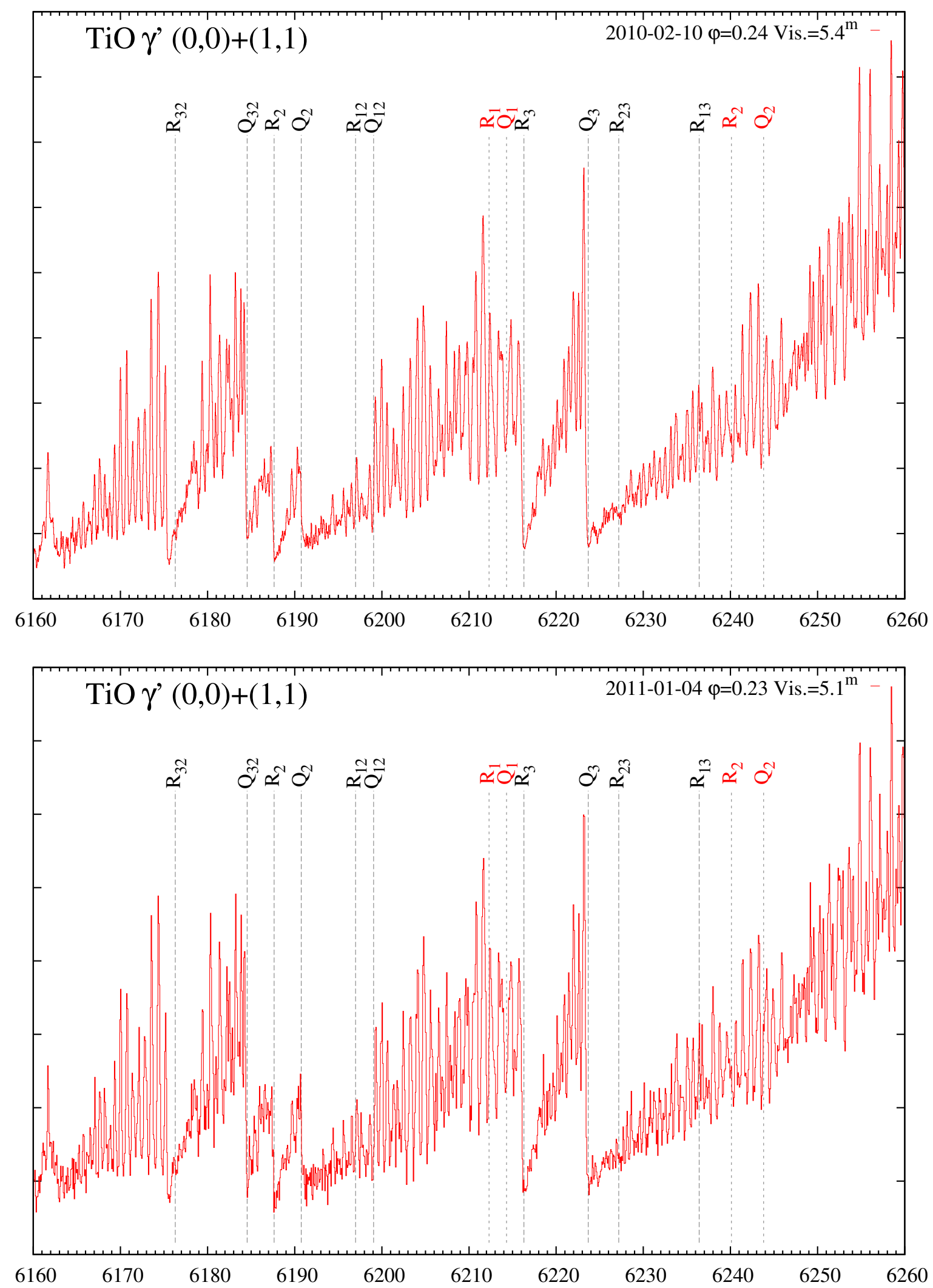

Fig. C.1. continued. 

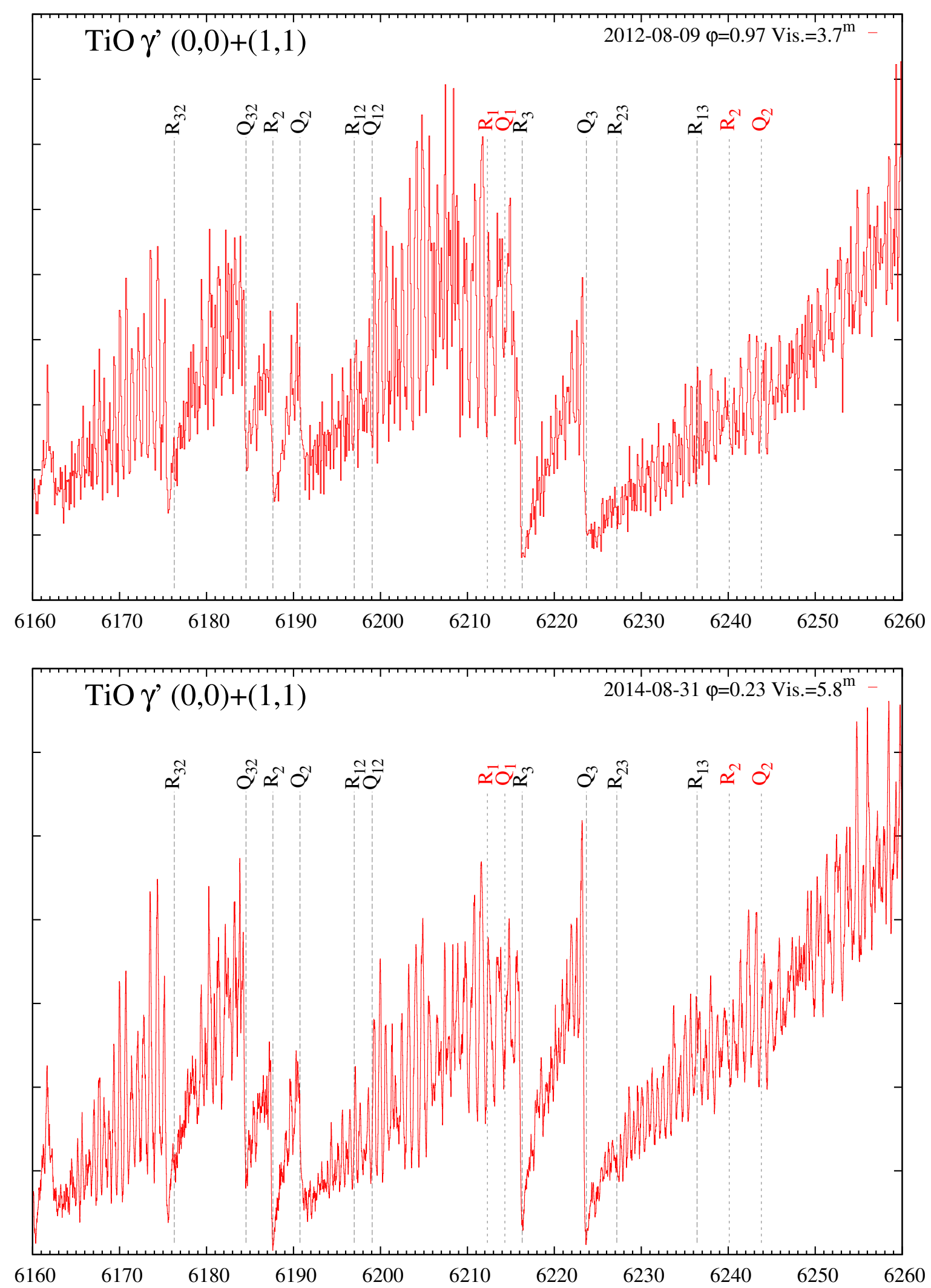

Fig. C.1. continued. 